\title{
Benchmarks for Higgs effective theory: extended Higgs sectors
}

\author{
Martin Gorbahn, ${ }^{a}$ Jose Miguel $\mathrm{No}^{b}$ and Verónica Sanz ${ }^{b}$ \\ ${ }^{a}$ Department of Mathematical Sciences, University of Liverpool, \\ Liverpool, L69 7ZL, U.K. \\ ${ }^{b}$ Department of Physics and Astronomy, University of Sussex, \\ Brighton, BN1 9QH, U.K. \\ E-mail: Martin.Gorbahn@liverpool.ac.uk, J.M.No@sussex.ac.uk, \\ v.sanz@sussex.ac.uk
}

ABSTRACT: Precise measurements of SM particles properties at the LHC allows to look for heavy New Physics in the context of an Effective Field Theory (EFT). These searches, however, often rely on kinematic regions where the validity of the EFT may be compromised. In this paper we propose to address this issue by comparing with benchmark models. The connection between models and their manifestations as EFTs at low energies allows us to quantify the breakdown of the EFT, and describe ways to combine different sources of constraints beyond Higgs physics. To illustrate these techniques, in this paper we propose a set of benchmark models based on extensions of the Higgs sector, namely the inclusion of a singlet, a dilaton and generic $2 \mathrm{HDM}$. We obtain the matching between these models and the EFT involving the Higgs, electroweak bosons and fermions. We then describe current and future indirect and direct constraints, consider the effect of correlations among the coefficients within models, and discuss the validity of the EFT.

Keywords: Higgs Physics, Beyond Standard Model, Effective field theories

ArXiv EPRINT: 1502.07352 


\section{Contents}

1 Introduction $\quad 1$

2 Effective field theory for the SM Higgs field 3

3 Higgs EFT from extended Higgs sectors 5

3.1 The singlet Higgs portal: doublet-singlet mixing 6

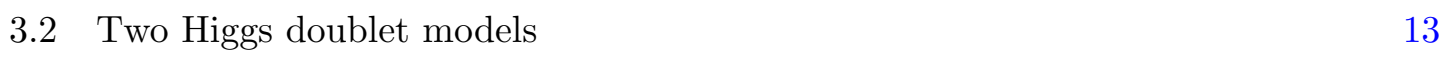

3.2.1 Benchmark A: exact alignment $c_{\beta-\alpha}=0 \quad 15$

3.2.2 Benchmark B: departure from alignment $c_{\beta-\alpha} \ll 1$ (MSSM-like) $\quad 16$

3.2.3 2HDM constraints: Higgs signal strengths \& EW oblique parameters 18

3.2.4 Alignment limit: complementarity of EWPTs and LHC limits 22

3.3 A dilaton/radion scenario 24

4 The validity of the EFT: from operators to total rates and distributions 28

$\begin{array}{llr}5 & \text { Discussion and summary } & 29\end{array}$

A 2HDM results and conventions $\quad 31$

A.1 Scalar potential and mass spectrum $\quad 31$

A.2 Electroweak precision observables 33

B Effective theory matching in the EW broken phase $\quad 34$

B.1 A specific example: 2HDM in the alignment limit $c_{\beta-\alpha}=0 \quad 38$

\section{Introduction}

The discovery of a Higgs boson [1, 2] has opened the Era of Higgs Physics. An intense effort is now devoted to measure the properties of this new particle $h$ and determine whether it is the Standard Model (SM) Higgs boson [3-6]. So far, no evidence for new physics beyond the SM has been observed, which suggests the use of an Effective Field Theory (EFT) approach, that assumes possible new physics beyond the SM modifying the Higgs interactions to be heavy, with a typical scale $\Lambda \sim \mathrm{TeV}$. The effects of new physics are then parametrized through higher-dimensional operators constructed from the SM fields, the leading operators appearing at dimension-six [7-12]. Some of these operators which affect Higgs properties can be measured in Higgs physics only, while others are also related to electroweak (EW) observables since the Higgs scalar excitation is always associated with the EW symmetry breaking order parameter $v$. The experimental data from LEP and Tevatron constrain the size of the Wilson coefficients of these operators, and more recently 
the results from the LHC experiments ATLAS and CMS allow also to constrain the Wilson coefficients associated to the operators that impact Higgs physics [13-21].

The LHC energy reach allows to go beyond the analysis of Higgs signal strengths as a probe of new physics beyond the SM, exploiting the information encoded in kinematical distributions to boost the sensitivity to new physics beyond the SM in the Higgs sector. This has the power to strongly constrain the presence of new physics which lead to a significant enhancement of the signal in certain kinematical regions, typically at high energy. However, while the bounds on the Wilson coefficients of these dimension-six operators which can be extracted from these measurements are indeed very strong even with the limited amount of present data, the validity of the EFT approach in the kinematical regions which dominate these constraints is questionable $[22,23]$ (see also [21]). This would render these constraints meaningless in the context of EFTs. It is therefore crucial to assess in detail the validity of this approach, and a way to do so is by quantitatively studying the breakdown of the EFT by comparing its predictions with those of possible UV completions.

Among possible UV completions, extended scalar sectors provide an attractive arena for the use of a SM effective theory description. Extensions of the SM Higgs sector constitute a simple yet very well-motivated scenario beyond the SM, with important consequences not only for phenomenology but also for EW cosmology, baryogenesis and dark matter. It is plausible that the new scalar states, which we assume to be significantly heavier than the Higgs boson discovered by ATLAS and CMS, cannot be detected directly at LHC either due to them being very heavy or having vanishingly small couplings to SM gauge bosons if the light Higgs is SM-like. However, it may be possible to measure the effect of these new particles via EW precision observables (EWPO) or using the kinematical information in LHC measurements. An EFT of extended Higgs sectors provides a very useful tool to study these effects systematically under the assumption that the new states are significantly heavier than $h$. Moreover, since extensions of the SM Higgs sector provide a very simple UV completion to such an EFT, this makes it possible to compare the predictions and bounds derived from the EFT with those of its UV completion, therefore probing the range of validity of the EFT. This allows us to assess the reliability of the LHC constraints drawn from the high-energy kinematical regions, as well as those from EWPO, and also to analyze the LHC potential for indirectly probing extended Higgs sectors.

The paper is organized as follows: in section 2 we introduce the $D=6$ effective operators which are relevant for the analysis. In section 3 we present the matching of these operators with UV models corresponding to extensions of the SM Higgs sector with an extra scalar singlet, with an extra scalar doublet (a Two-Higgs-Doublet-Model) and with a radion/dilaton, and discuss their experimental constraints both from the UV model and from the EFT point of view. In section 4 we discuss the validity of the EFT in the light of these scenarios. Finally, in section 5 we summarize our results and discuss their implications. In the appendices we describe the connection to the various possible $D=6$ Lagrangian terms after EW symmetry breaking, parametrized as anomalous Higgs couplings, and give details on the $2 \mathrm{HDM}$ relevant to our analysis. 


\section{Effective field theory for the SM Higgs field}

The Lagrangian for the Standard Model can be supplemented by higher-dimensional operators that parametrize the effects of new physics beyond the SM appearing at energies much larger than an effective scale which may identified with the vev of the Higgs field $v$. Considering only operators of dimension $D \leq 6$ and assuming baryon and lepton number conservation, the most general $\mathrm{SU}(3)_{C} \times \mathrm{SU}(2)_{L} \times \mathrm{U}(1)_{Y}$ gauge invariant Lagrangian $\mathcal{L}_{\text {Eff }}$ constructed out of the SM fields has been known for a long time [7-9], and may be mapped into various alternative bases of $D \leq 6$ independent SM effective operators [10-12] (see also the discussion in [24]).

Each experimental measurement will in general constrain only a handful of effective operators of a certain basis. In combining the available constraints from analyses of various physical processes (via e.g. a global fit), some basis choices will be more appropriate than others. ${ }^{1}$ As compared to those of $[10,12]$, the basis of operators $\mathcal{O}_{i}$ from [11] is wellsuited for analyses of Higgs properties in combination with precision measurements of EW observables. In this basis, the SM effective Lagrangian $\mathcal{L}_{\text {Eff }}$ may be expressed as

$$
\mathcal{L}_{\mathrm{Eff}}=\mathcal{L}_{\mathrm{SM}}+\sum_{i} \bar{c}_{i} \mathcal{O}_{i}=\mathcal{L}_{\mathrm{SM}}+\mathcal{L}_{\mathrm{SILH}}+\mathcal{L}_{G}+\mathcal{L}_{C P}+\mathcal{L}_{F_{1}}+\mathcal{L}_{F_{2}}+\mathcal{L}_{F_{3}}
$$

We adopt here the decomposition in $[25,26]$ and normalize the Wilson coefficients $\bar{c}_{i}$ accordingly. The term $\mathcal{L}_{\mathrm{SILH}}$ of $(2.1)$ corresponds to a certain set of $C P$-conserving operators involving the Higgs doublet $\Phi$, inspired by scenarios where the Higgs is part of a strongly interacting sector [11]

$$
\begin{aligned}
\mathcal{L}_{\mathrm{SILH}}= & \frac{\bar{c}_{H}}{2 v^{2}} \partial^{\mu}\left[\Phi^{\dagger} \Phi\right] \partial_{\mu}\left[\Phi^{\dagger} \Phi\right]+\frac{\bar{c}_{T}}{2 v^{2}}\left[\Phi^{\dagger} \overleftrightarrow{D}^{\mu} \Phi\right]\left[\Phi^{\dagger} \overleftrightarrow{D}_{\mu} \Phi\right]-\frac{\bar{c}_{6} \lambda}{v^{2}}\left[\Phi^{\dagger} \Phi\right]^{3} \\
& +\frac{i g \bar{c}_{W}}{m_{W}^{2}}\left[\Phi^{\dagger} T_{2 k} \overleftrightarrow{D}^{\mu} \Phi\right] D^{\nu} W_{\mu \nu}^{k}+\frac{i g^{\prime} \bar{c}_{B}}{2 m_{W}^{2}}\left[\Phi^{\dagger} \overleftrightarrow{D}^{\mu} \Phi\right] \partial^{\nu} B_{\mu \nu} \\
& +\frac{2 i g \bar{c}_{H W}}{m_{W}^{2}}\left[D^{\mu} \Phi^{\dagger} T_{2 k} D^{\nu} \Phi\right] W_{\mu \nu}^{k}+\frac{i g^{\prime} \bar{c}_{H B}}{m_{W}^{2}}\left[D^{\mu} \Phi^{\dagger} D^{\nu} \Phi\right] B_{\mu \nu} \\
& +\frac{g^{\prime 2} \bar{c}_{\gamma}}{m_{W}^{2}} \Phi^{\dagger} \Phi B_{\mu \nu} B^{\mu \nu}+\frac{g_{s}^{2} \bar{c}_{g}}{m_{W}^{2}} \Phi^{\dagger} \Phi G_{\mu \nu}^{a} G_{a}^{\mu \nu} \\
& -\left[\frac{\bar{c}_{u} y_{u}}{v^{2}} \Phi^{\dagger} \Phi \bar{Q}_{L} \Phi^{\dagger} u_{R}+\frac{\bar{c}_{d} y_{d}}{v^{2}} \Phi^{\dagger} \Phi \bar{Q}_{L} \Phi d_{R}+\frac{\bar{c}_{l} y_{l}}{v^{2}} \Phi^{\dagger} \Phi \bar{L}_{L} \Phi l_{R}\right]
\end{aligned}
$$

Here $\lambda$ stands for the Higgs quartic coupling, $g^{\prime}, g$ and $g_{s}$ are respectively the $\mathrm{U}(1)_{Y}$, $\mathrm{SU}(2)_{L}$ and $\mathrm{SU}(3)_{C}$ coupling constants and $T_{2 k} \equiv \sigma_{k} / 2$ are the generators of $\mathrm{SU}(2)$ in the fundamental representation ( $\sigma_{k}$ being the Pauli matrices). The Hermitian derivative operator $\overleftrightarrow{D}_{\mu}$ is defined as

$$
\Phi^{\dagger} \overleftrightarrow{D}_{\mu} \Phi=\Phi^{\dagger} D_{\mu} \Phi-\left(D_{\mu} \Phi^{\dagger}\right) \Phi
$$

and our conventions for the gauge-covariant derivatives and field-strength tensors (following [26]) are

$$
B_{\mu \nu}=\partial_{\mu} B_{\nu}-\partial_{\nu} B_{\mu}
$$

\footnotetext{
${ }^{1}$ Depending on the purposes, bases different from the one we adopt here might be more adequate.
} 


$$
\begin{aligned}
W_{\mu \nu}^{k}= & \partial_{\mu} W_{\nu}^{k}-\partial_{\nu} W_{\mu}^{k}+g \epsilon_{i j}{ }^{k} W_{\mu}^{i} W_{\nu}^{j} \\
G_{\mu \nu}^{a}= & \partial_{\mu} G_{\nu}^{a}-\partial_{\nu} G_{\mu}^{a}+g_{s} f_{b c}{ }^{a} G_{\mu}^{b} G_{\nu}^{c} \\
D_{\mu} \Phi= & \partial_{\mu} \Phi-i g T_{2 k} W_{\mu}^{k} \Phi-\frac{i}{2} g^{\prime} B_{\mu} \Phi \\
D_{\rho} W_{\mu \nu}^{k}= & \partial_{\rho} \partial_{\mu} W_{\nu}^{k}-\partial_{\rho} \partial_{\nu} W_{\mu}^{k}+g \epsilon_{i j}{ }^{k} \partial_{\rho}\left(W_{\mu}^{i} W_{\nu}^{j}\right) \\
& +g \epsilon_{i j}{ }^{k} W_{\rho}^{i}\left(\partial_{\mu} W_{\nu}^{j}-\partial_{\nu} W_{\mu}^{j}\right)-g^{2} W_{\rho i}\left(W_{\mu}^{i} W_{\nu}^{k}-W_{\nu}^{i} W_{\mu}^{k}\right) \\
D_{\rho} G_{\mu \nu}^{a}= & \partial_{\rho} \partial_{\mu} G_{\nu}^{a}-\partial_{\rho} \partial_{\nu} G_{\mu}^{a}+g_{s} f_{b c}{ }^{a} \partial_{\rho}\left(G_{\mu}^{b} G_{\nu}^{c}\right) \\
& +g_{s} f_{b c}{ }^{a} G_{\rho}^{b}\left(\partial_{\mu} G_{\nu}^{c}-\partial_{\nu} G_{\mu}^{c}\right)-g_{s}^{2} G_{\rho b}\left(G_{\mu}^{b} G_{\nu}^{a}-G_{\nu}^{b} G_{\mu}^{a}\right),
\end{aligned}
$$

with $\epsilon_{i j}{ }^{k}$ and $f_{b c}{ }^{a}$ being respectively the structure constants for $\mathrm{SU}(2)$ and $\mathrm{SU}(3)$. The term $\mathcal{L}_{\mathrm{G}}$ consists of operators not directly connected to Higgs physics, but that affect the gauge sector through modifications of the gauge boson self-energies and self-interactions,

$$
\begin{aligned}
\mathcal{L}_{G}= & \frac{g^{3} \bar{c}_{3 W}}{m_{W}^{2}} \epsilon_{i j k} W_{\mu \nu}^{i} W_{\rho}^{\nu j} W^{\rho \mu k}+\frac{g_{s}^{3} \bar{c}_{3 G}}{m_{W}^{2}} f_{a b c} G_{\mu \nu}^{a} G_{\rho}^{\nu b} G^{\rho \mu c}+\frac{g^{2} \bar{c}_{2 W}}{m_{W}^{2}} D^{\mu} W_{\mu \nu}^{k} D_{\rho} W_{k}^{\rho \nu} \\
& +\frac{g^{\prime 2} \bar{c}_{2 B}}{m_{W}^{2}} \partial^{\mu} B_{\mu \nu} \partial_{\rho} B^{\rho \nu}+\frac{g_{s}^{2} \bar{c}_{2 G}}{m_{W}^{2}} D^{\mu} G_{\mu \nu}^{a} D_{\rho} G_{a}^{\rho \nu} .
\end{aligned}
$$

The term $\mathcal{L}_{\mathrm{CP}}$ in $(2.1)$ supplements $\mathcal{L}_{\mathrm{SILH}}$ and $\mathcal{L}_{G}$ with a set of CP-violating operators

$$
\begin{aligned}
\mathcal{L}_{C P}= & \frac{i g \tilde{c}_{H W}}{m_{W}^{2}} D^{\mu} \Phi^{\dagger} T_{2 k} D^{\nu} \Phi \widetilde{W}_{\mu \nu}^{k}+\frac{i g^{\prime} \tilde{c}_{H B}}{m_{W}^{2}} D^{\mu} \Phi^{\dagger} D^{\nu} \Phi \widetilde{B}_{\mu \nu}+\frac{g^{\prime 2} \tilde{c}_{\gamma}}{m_{W}^{2}} \Phi^{\dagger} \Phi B_{\mu \nu} \widetilde{B}^{\mu \nu} \\
& +\frac{g_{s}^{2} \tilde{c}_{g}}{m_{W}^{2}} \Phi^{\dagger} \Phi G_{\mu \nu}^{a} \widetilde{G}_{a}^{\mu \nu}+\frac{g^{3} \tilde{c}_{3 W}}{m_{W}^{2}} \epsilon_{i j k} W_{\mu \nu}^{i} W_{\rho}^{\nu j} \widetilde{W}^{\rho \mu k}+\frac{g_{s}^{3} \tilde{c}_{3 G}}{m_{W}^{2}} f_{a b c} G_{\mu \nu}^{a} G_{\rho}^{\nu b} \widetilde{G}^{\rho \mu c},
\end{aligned}
$$

with the dual field strength tensors defined by

$$
\widetilde{B}_{\mu \nu}=\frac{1}{2} \epsilon_{\mu \nu \rho \sigma} B^{\rho \sigma}, \quad \widetilde{W}_{\mu \nu}^{k}=\frac{1}{2} \epsilon_{\mu \nu \rho \sigma} W^{\rho \sigma k}, \quad \widetilde{G}_{\mu \nu}^{a}=\frac{1}{2} \epsilon_{\mu \nu \rho \sigma} G^{\rho \sigma a} .
$$

Finally, there are further operators contained in $\mathcal{L}_{F_{1}}$-operators involving two Higgs fields and a pair of quarks/leptons-, $\mathcal{L}_{F_{2}}$-operators involving one Higgs field, a gauge boson and a pair of quarks/leptons- and $\mathcal{L}_{F_{3}}$-four-fermion operators- (see e.g. [25, 26]). These effective operators are nevertheless not present at leading order in the extensions of the $\mathrm{SM}$ we consider in the present work, ${ }^{2}$ and so we do not discuss them in the following. Furthermore, we consider CP conserving scenarios for the time being, leaving an analysis of $\mathcal{L}_{C P}$ for the future [27].

After EW symmetry breaking, we can write the SM effective Lagrangian $\mathcal{L}_{\text {Eff }}$ in the unitarity gauge and in the mass basis, with

$$
W_{\mu}^{ \pm}=\frac{1}{\sqrt{2}}\left(W_{\mu}^{1} \mp i W_{\mu}^{2}\right) \quad\left(\begin{array}{c}
Z_{\mu} \\
A_{\mu}
\end{array}\right)=\left(\begin{array}{rr}
c_{W} & -s_{W} \\
s_{W} & c_{W}
\end{array}\right)\left(\begin{array}{c}
W_{\mu}^{3} \\
B_{\mu}
\end{array}\right) \quad \Phi=\frac{1}{\sqrt{2}}\left(\begin{array}{c}
0 \\
v+h
\end{array}\right)
$$

\footnotetext{
${ }^{2}$ An exception are certain operators in $\mathcal{L}_{F_{3}}$ which do get generated in Two-Higgs-Doublet-Model extensions of the SM. These are however proportional to $Y_{a}^{2}$ (with $Y_{a}$ a fermion Yukawa coupling), being then negligible for $1^{\text {st }}$ and $2^{\text {nd }}$ fermion generations, and so are essentially unconstrained.
} 
with $s_{W}\left(c_{W}\right)$ being the sine (cosine) of the weak mixing angle at tree level. In appendix B, we detail the relation of the $D=6$ terms presented here with the possible anomalous Higgs couplings after EW symmetry breaking.

We are now ready to move onto relating the EFT to specific UV completions. In this work we have chosen to focus on the matching of the Wilson coefficients onto models with extended Higgs sectors, where the effects of New Physics are more apparent in deviations of the couplings of the Higgs and electroweak bosons. We describe the results of the matching, as well as the relation between these models and the EFT in the next section.

\section{Higgs EFT from extended Higgs sectors}

As discussed in the Introduction, an important aspect of the $D \leq 6$ SM effective theory from the previous section is its energy range of validity. This is a key issue if one is to reliably confront the predictions of the effective theory with experimental data, in particular those sensitive to energy scales $E \gg v$. In this sense, extended (non-minimal) scalar sectors provide a very simple renormalizable completion to the $D \leq 6$ SM effective theory, and allow for a quantitative assessment of the EFT's energy range of validity.

Moreover, extensions of the SM scalar sector provide an attractive arena for the use of the SM effective theory: they are a simple scenario beyond the SM, well-motivated from the point of view of EW cosmology and baryogenesis, and may also be regarded as part of a complete theory beyond the Standard Model at the TeV scale, such as Composite Higgs scenarios or low-energy Supersymmetry. Assuming that the new scalar states are significantly heavier than $m_{h}$, the effective theory of extended Higgs sectors provides a way to study these effects systematically, as has already been shown e.g. in [32]. In particular, it is plausible that the new scalar states from the extended Higgs sector are very hard to probe at LHC (they might be very heavy, or have no decay branching fractions to SM gauge bosons), but their effect might be possible to detect either in precision EW measurements or using differential information in LHC measurements.

Below we construct the $D \leq 6 \mathrm{SM}$ effective theory for various extensions of the SM scalar sector: a singlet extension of the SM (the so-called "Higgs portal"), a Two Higgs Doublet Model and an extension of the SM by a dilaton/radion. In each case, we obtain the Wilson coefficients for the $D=6$ effective operators by matching to the UV theory, and perform an analysis of their current experimental bounds and future prospects. The results of this construction for the different scenarios is briefly summarized in table 1 .

In the respective matching procedure we demand that the effective action for the UV theory and the $D \leq 6$ EFT agree after an expansion in the light degrees of freedoms over the new physics mass scale. This results in the matching of the one-light-particle irreducible (1LPI) Green's functions in the full and effective theory — see [33] for a pedagogical introduction for these type of calculations. In the following we will perform an off-shell matching, where we expand in external momenta and the EW mass-scale over the new physics mass scale and keep equation of motion vanishing operators in the calculation until the final projection. Accordingly all propagators of SM fields will be massless after the expansion and we can perform the calculation in the $\mathrm{SU}(2)_{L} \times \mathrm{U}(1)_{Y}$ symmetric phase, 


\begin{tabular}{|c|c|c|c|c|c|c|c|c|c|c|}
\hline & $\bar{c}_{H}$ & $\bar{c}_{6}$ & $\bar{c}_{T}$ & $\bar{c}_{W}$ & $\bar{c}_{B}$ & $\bar{c}_{H W}$ & $\bar{c}_{H B}$ & $\bar{c}_{3 W}$ & $\bar{c}_{\gamma}$ & $\bar{c}_{g}$ \\
\hline Higgs Portal $(G)$ & $\mathrm{L}$ & $\mathrm{L}$ & $\mathrm{X}$ & $\mathrm{X}$ & $\mathrm{X}$ & $\mathrm{X}$ & $\mathrm{X}$ & $\mathrm{X}$ & $\mathrm{X}$ & $\mathrm{X}$ \\
\hline Higgs Portal (Spontaneous $Q^{7}$ ) & $\mathrm{T}$ & $\mathrm{L}$ & $\mathrm{RG}$ & $\mathrm{RG}$ & $\mathrm{RG}$ & $\mathrm{X}$ & $\mathrm{X}$ & $\mathrm{X}$ & $\mathrm{X}$ & $\mathrm{X}$ \\
\hline Higgs Portal (Explicit $\left.Q^{*}\right)$ & $\mathrm{T}$ & $\mathrm{T}$ & $\mathrm{RG}$ & $\mathrm{RG}$ & RG & $\mathrm{X}$ & $\mathrm{X}$ & $\mathrm{X}$ & $\mathrm{X}$ & $\mathrm{X}$ \\
\hline $2 \mathrm{HDM}$ Benchmark A $\left(c_{\beta-\alpha}=0\right)$ & $\mathrm{L}$ & $\mathrm{L}$ & $\mathrm{L}$ & $\mathrm{L}$ & $\mathrm{L}$ & $\mathrm{L}$ & $\mathrm{L}$ & $\mathrm{L}$ & $\mathrm{L}$ & $\mathrm{X}$ \\
\hline 2HDM Benchmark B $\left(c_{\beta-\alpha} \neq 0\right)$ & $\mathrm{T}$ & $\mathrm{T}$ & $\mathrm{L}$ & $\mathrm{L}$ & $\mathrm{L}$ & $\mathrm{L}$ & $\mathrm{L}$ & $\mathrm{L}$ & $\mathrm{L}$ & $\mathrm{X}$ \\
\hline Radion/Dilaton & $\mathrm{T}$ & $\mathrm{T}$ & RG & $\mathrm{T}$ & $\mathrm{T}$ & $\mathrm{T}$ & $\mathrm{T}$ & $\mathrm{L}$ & $\mathrm{T}$ & $\mathrm{T}$ \\
\hline
\end{tabular}

Table 1. Leading order at which the various Wilson coefficients for the $D=6 \mathrm{SM}$ effective field theory are generated in each of the scenarios under consideration. In each case, the operator can be generated at Tree-Level (T) or 1-Loop (L). If some operators are generated at Tree-Level, this may lead to the generation of others via operator mixing under 1-loop Renormalization Group evolution (see e.g. [28-31]), which we denote by RG. Operators which are generated at higher order in RG and EFT expansion are denoted with an X.

quite analogous to the matching calculation performed in [34]. The light degrees of freedoms of the $\mathrm{SU}(2)_{L} \times \mathrm{U}(1)_{Y}$ symmetric phase comprise the gauge singlet $B$, triplet $W^{a}$ and octet $G^{a}$ fields as well as the scalar doublet $\Phi$ and the fermionic doublets and singlets. In practice we compute 1PI Green's functions with up to 6 Higgs and 3 gauge boson fields where the total number of fields does not exceed 8 . The resulting expressions are obviously related via $\mathrm{SU}(2)_{L} \times \mathrm{U}(1)_{Y}$ gauge invariance of the operators in (2.2), (2.5), (2.6) and (3.40), which provides a useful consistency check of our calculation. Moreover, we do cross-check with an explicit calculation in the broken phase, detailed in appendix B.

\subsection{The singlet Higgs portal: doublet-singlet mixing}

The addition of a singlet (real or complex) scalar field is arguably the simplest possible extension of the SM. Despite its minimality, this extension of the SM can have important consequences for the stability of the EW vacuum at high energies $[35,36]$, and it could at the same time constitute a "Higgs portal" into a dark/hidden sector $[37,38]$. It may also have important consequences for Cosmology, potentially accounting for the dark matter relic density [39-41] or yielding a first order EW phase transition in the early Universe [42-49] that could explain the matter-antimatter asymmetry in the Universe through baryogenesis. In addition, it may give rise to interesting collider phenomenology (see e.g. [50-53]). Altogether, the singlet scalar extension of the SM constitutes a well-motivated scenario, and the interplay of different present and future experimental data to probe it has been widely studied (see [54-57] for up-to-date analyses). Let us then consider the SM scalar potential extended by a singlet scalar field $s$

$$
V(\Phi, s)=-\mu_{\mathrm{H}}^{2}|\Phi|^{2}+\lambda|\Phi|^{4}-\frac{\mu_{\mathrm{S}}^{2}}{2} s^{2}+\frac{\lambda_{\mathrm{S}}}{4} s^{4}+\frac{\lambda_{\mathrm{m}}}{2}|\Phi|^{2} s^{2} .
$$


We assume initially that linear and cubic terms in $s$ are absent from $V(\Phi, s)$, which may be achieved by means of a discrete/continuous symmetry $G$ in the hidden sector, and focus on the scenario in which the field $s$ develops a vev, ${ }^{3} s \rightarrow s+v_{s}$. This generates linear and cubic terms in $s$, which specific relations among these and the rest of parameters in the potential. After EW symmetry breaking, the scalar potential reads

$$
\begin{aligned}
V(h, s)= & \frac{m_{h}^{2}}{2} h^{2}+\frac{m_{s}^{2}}{2} s^{2}+m_{h s}^{2} h s+v \lambda h^{3}+v_{s} \lambda_{\mathrm{S}} s^{3}+\frac{\lambda_{\mathrm{m}} v}{2} h s^{2}+\frac{\lambda_{\mathrm{m}} v_{s}}{2} h^{2} s \\
& +\frac{\lambda_{\mathrm{m}}}{4} h^{2} s^{2}+\frac{\lambda}{4} h^{4}+\frac{\lambda_{\mathrm{S}}}{4} s^{4}
\end{aligned}
$$

with $m_{h}^{2}=2 \lambda v^{2}, m_{s}^{2}=2 \lambda_{\mathrm{S}} v_{s}^{2}$ and $m_{h s}^{2}=\lambda_{\mathrm{m}} v v_{s}$, and where we have used the minimization conditions $\mu_{\mathrm{H}}^{2}=\lambda v^{2}+\frac{\lambda_{\mathrm{m}}}{2} v_{s}^{2}$ and $\mu_{\mathrm{S}}^{2}=\lambda_{\mathrm{S}} v_{s}^{2}+\frac{\lambda_{\mathrm{m}}}{2} v^{2}$ in $V(h, s)$ to trade the mass parameters $\mu_{\mathrm{H}}^{2}, \mu_{\mathrm{S}}^{2}$ for the vevs. The term $m_{h s}^{2} h s$ in (3.2) induces doublet-singlet mixing, leading to two mass eigenstates $h_{1,2}$, the lighter of which $\left(h_{1}\right)$ we identify with the discovered $125 \mathrm{GeV}$ Higgs particle. The mixing angle $\theta$ and masses are given by

$$
\begin{aligned}
s_{\theta}^{2} & =\frac{4 m_{h s}^{4}}{4 m_{h s}^{4}+\left(m_{s}^{2}-m_{h}^{2}+\sqrt{\left(m_{s}^{2}-m_{h}^{2}\right)^{2}+4 m_{h s}^{4}}\right)^{2}}=\frac{4 y^{2}}{4 y^{2}+\left(1-x^{2}+\sqrt{\left(1-x^{2}\right)^{2}+4 y^{2}}\right)^{2}} \\
m_{1,2}^{2} & =\frac{1}{2}\left(m_{h}^{2}+m_{s}^{2} \mp \sqrt{\left(m_{s}^{2}-m_{h}^{2}\right)^{2}+4 m_{h s}^{4}}\right)=\frac{m_{s}^{2}}{2}\left(1+x^{2} \mp \sqrt{\left(1-x^{2}\right)^{2}+4 y^{2}}\right)
\end{aligned}
$$

with $s_{\theta} \equiv \sin (\theta), x \equiv m_{h} / m_{s} \sim v / v_{s}$ and $y \equiv m_{h s}^{2} / m_{s}^{2} \sim v / v_{s}$. The limit $m_{s} \gg v$ corresponds then to $v_{s} \gg v$, with $x, y \ll 1$ and so

$$
s_{\theta}^{2} \simeq y^{2} \quad, \quad m_{1}^{2} \simeq m_{s}^{2}\left(x^{2}-y^{2}\right)=m_{h}^{2}-s_{\theta}^{2} m_{s}^{2} \quad, \quad m_{2}^{2} \simeq m_{s}^{2}\left(1+y^{2}\right)=m_{s}^{2}\left(1+s_{\theta}^{2}\right)
$$

where we have neglected terms of $\mathcal{O}\left(x^{4}, x^{2} y^{2}, y^{4}\right)$. From (3.2), the relevant scalar selfinteractions $h_{1}^{2} h_{2}$ and $h_{1}^{3}$ read

$$
V\left(h_{1}, h_{2}\right) \supset\left[\frac{m_{1}^{2}}{2 v}+\mathcal{O}\left(x^{2}, y^{2}\right)\right] h_{1}^{3}+\left[\frac{m_{s}^{2} y}{2 v}+\mathcal{O}(x, y)\right] h_{1}^{2} h_{2}
$$

Neglecting $\mathcal{O}\left(y^{4}\right)$ corrections, the couplings of $h_{1,2}$ to the $W^{ \pm}$and $Z$ bosons read

$$
\begin{aligned}
& {\left[g m_{W}\left(1-y^{2} / 2\right) h_{1}+\frac{g^{2}}{4}\left(1-y^{2}\right) h_{1}^{2}-\left(g m_{W} y\right) h_{2}+\ldots\right] W_{\mu}^{+} W^{\mu-}} \\
& +\left[\frac{g m_{Z}}{2 c_{\mathrm{W}}}\left(1-y^{2} / 2\right) h_{1}+\frac{g^{2}}{8 c_{\mathrm{W}}^{2}}\left(1-y^{2}\right) h_{1}^{2}-\left(\frac{g m_{Z}}{2 c_{\mathrm{W}}} y\right) h_{2}+\ldots\right] Z_{\mu} Z^{\mu}
\end{aligned}
$$

Noting that $y \equiv m_{h s}^{2} / m_{s}^{2}=m_{h s}^{2} / m_{2}^{2}+\mathcal{O}\left(y^{3}\right)$, we may integrate out the heavy, singletlike state $h_{2}$. At leading order, this generates an $\mathcal{O}\left(y^{2}\right)$ contribution to $h_{1}^{2} V_{\mu} V^{\mu}$, which precisely cancels the $\mathcal{O}\left(y^{2}\right)$ correction in (3.7). The Higgs-gauge interactions then read

$$
\left[g m_{W}\left(1-y^{2} / 2\right) h_{1}+\frac{g^{2}}{4} h_{1}^{2}\right] W_{\mu}^{+} W^{\mu-}+\left[\frac{g m_{Z}}{2 c_{\mathrm{W}}}\left(1-y^{2} / 2\right) h_{1}+\frac{g^{2}}{8 c_{\mathrm{W}}^{2}} h_{1}^{2}\right] Z_{\mu} Z^{\mu},
$$

\footnotetext{
${ }^{3}$ The spontaneous breaking of a discrete or global continuous symmetry $G$ would respectively lead to domain wall formation in the early Universe or the existence of massless Goldstone bosons, both features being undesirable in a realistic model. Possible solutions are to consider $G$ to be a spontaneously broken gauge symmetry, or to allow for a small explicit breaking of the symmetry. We will disregard these issues in the following discussion.
} 

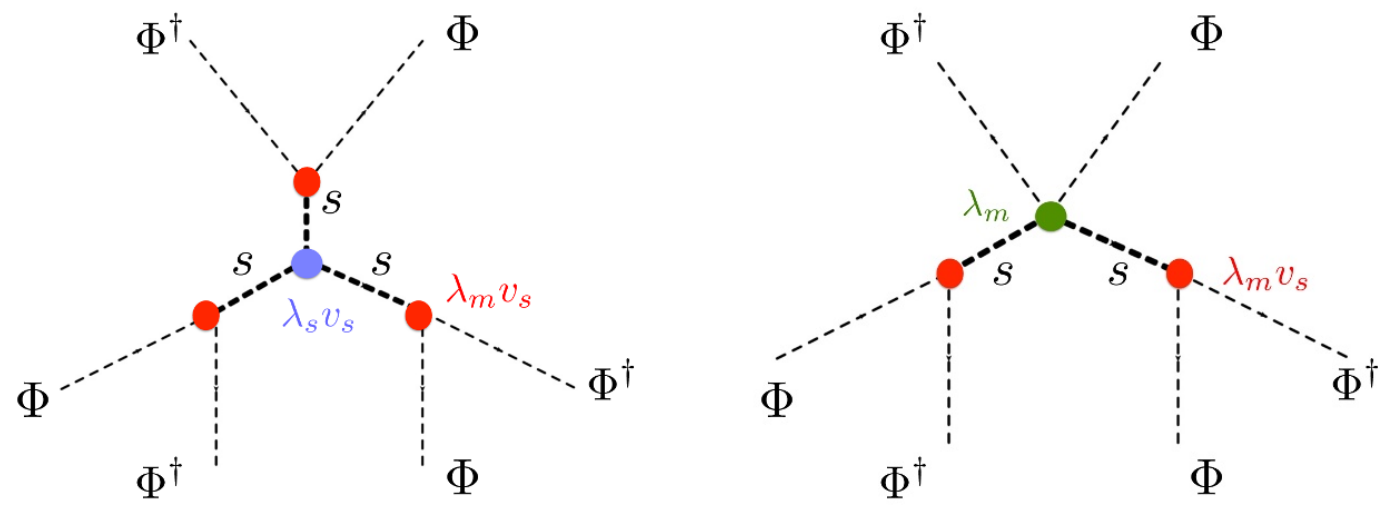

Figure 1. The two Feynman diagrams leading to the effective operator $\bar{c}_{6}$ in the singlet extension of the SM. Their respective combinatorial factors are 6 (Left) and 3 (Right).

leading to a $g_{V}^{2} y^{2} / 2$ mismatch (with $g_{V}=\frac{g}{\sqrt{2}}, \frac{g}{\sqrt{2} c_{W}}$ for $V=W, Z$ respectively) between $g_{h h V V}^{(3)}$ and $\frac{g g_{h V V}^{(3)}}{2 m_{W}}$. We turn now to the fermionic couplings, focusing on the Higgs-top quark interactions

$$
\frac{Y_{t}}{\sqrt{2}}\left(1-\frac{y^{2}}{2}\right) h_{1} \bar{t}_{L} t_{R}-\frac{Y_{t} y}{\sqrt{2}} h_{2} \bar{t}_{L} t_{R}
$$

with $Y_{t}$ the top-quark Yukawa coupling. This affects the gluon fusion Higgs effective coupling for $h_{1}$, which gets modified w.r.t. the SM one

$$
-\frac{g_{h g g}}{4}\left(1-y^{2} / 2\right) G_{\mu \nu}^{a} G_{a}^{\mu \nu} h_{1} .
$$

The coupling $G_{\mu \nu}^{a} G_{a}^{\mu \nu} h_{1}^{2}$ (relevant for di-Higgs production) also gets modified, both through an $\mathcal{O}\left(y^{2}\right)$ correction due to the singlet-doublet mixing and directly via the presence of $h_{2}$, which can mediate the process $g g \rightarrow h_{1} h_{1}$ (for $\hat{\mathrm{s}} / m_{s}^{2} \ll 1$, being $\hat{\mathrm{s}}$ the partonic center of mass energy for the process, this contribution is however small).

The above discussion may be directly mapped into an $\mathrm{SU}(2)_{L} \times \mathrm{U}(1)_{Y}$ invariant effective field theory for the SM. We consider (3.1) after $s$ develops a vev

$$
V(\Phi, s)=-\tilde{\mu}_{\mathrm{H}}^{2}|\Phi|^{2}+\lambda|\Phi|^{4}+\frac{m_{s}^{2}}{2} s^{2}+v_{s} \lambda_{\mathrm{S}} s^{3}+\frac{\lambda_{\mathrm{S}}}{4} s^{4}+\lambda_{\mathrm{m}} v_{s}|\Phi|^{2} s+\frac{\lambda_{\mathrm{m}}}{2}|\Phi|^{2} s^{2},
$$

with $\tilde{\mu}_{\mathrm{H}}^{2}=\mu_{\mathrm{H}}^{2}-\left(\lambda_{\mathrm{m}} v_{s}^{2}\right) / 2$. We then integrate-out the field $s$, which yields the effective Lagrangian

$$
\mathcal{L}_{\mathrm{Eff}} \supset \frac{\lambda_{\mathrm{m}}^{2} v_{s}^{2}}{2 m_{s}^{4}} \partial^{\mu}|\Phi|^{2} \partial_{\mu}|\Phi|^{2}-\left(6 \frac{\lambda_{\mathrm{m}}^{3} \lambda_{\mathrm{s}} v_{s}^{4}}{m_{s}^{6}}-3 \frac{\lambda_{\mathrm{m}}^{3} v_{s}^{2}}{m_{s}^{4}}\right)|\Phi|^{6}=\frac{y^{2}}{2 v^{2}} \partial^{\mu}|\Phi|^{2} \partial_{\mu}|\Phi|^{2} .
$$

From (2.2), this corresponds to $\bar{c}_{H}=y^{2}$. The two contributions to $\bar{c}_{6}$ are shown in figure 1 , and cancel by means of $m_{s}^{2}=2 \lambda_{\mathrm{S}} v_{s}^{2}$ (yielding $\bar{c}_{6}=0$ ). Upon EW symmetry breaking, $\bar{c}_{H}$ modifies the wave function of the Higgs $h$

$$
\mathcal{L}_{\text {Eff }} \supset\left(1+\bar{c}_{H}\right) \frac{1}{2}\left(\partial_{\mu} h\right)^{2}
$$


which universally reduces all couplings of $h$ : e.g. the coupling between $h$ and the weak bosons simply read

$$
g_{h V V}^{(3)}=\frac{g_{V}^{2} v}{\sqrt{1+y^{2}}} \simeq g_{V}^{2} v\left(1-\frac{y^{2}}{2}\right),
$$

matching as expected the result from (3.8).

The previous results for $\bar{c}_{H}$ are left unchanged by the inclusion of explicit linear and cubic terms for $s$ in (3.1). These can however generate a non-zero value for $\bar{c}_{6}$, since now the cancellation among the diagrams in figure 1 is not exact. Let us illustrate this by adding a term $\mu_{m}|\Phi|^{2} s$ to (3.11). This term does not alter the minimization conditions, but contributes to the singlet-doublet mixing upon EW symmetry breaking, so that now the mixing is given by $y \equiv m_{h s}^{2} / m_{s}^{2}=\left(\lambda_{\mathrm{m}} v_{s}+\mu_{m}\right) v / m_{s}^{2}$. Upon integrating-out $s$, this now yields

$$
\begin{aligned}
\mathcal{L}_{\text {Eff }} & \supset \frac{\left(\lambda_{\mathrm{m}} v_{s}+\mu_{m}\right)^{2}}{2 m_{s}^{4}} \partial^{\mu}|\Phi|^{2} \partial_{\mu}|\Phi|^{2}-\left(6 \frac{\left(\lambda_{\mathrm{m}} v_{s}+\mu_{m}\right)^{3} \lambda_{\mathrm{S}} v_{s}}{m_{s}^{6}}-3 \frac{\lambda_{\mathrm{m}}\left(\lambda_{\mathrm{m}} v_{s}+\mu_{m}\right)^{2}}{m_{s}^{4}}\right)|\Phi|^{6} \\
& =\frac{y^{2}}{2 v^{2}} \partial^{\mu}|\Phi|^{2} \partial_{\mu}|\Phi|^{2}-\frac{3 \mu_{m}}{v_{s}} \frac{y^{2}}{v^{2}}|\Phi|^{6}
\end{aligned}
$$

leading again to $\bar{c}_{H}=y^{2}$, and now to $\lambda \bar{c}_{6}=3 y^{2} \mu_{m} / v_{s}$. The parameter $\mu_{m} / v_{s} \equiv \delta$ measures the relative importance of explicit vs. spontaneous symmetry breaking in (3.1).

Let us also comment on the case where the field $s$ does not develop a vev and still the terms linear and cubic in $s$ are absent from (3.1) (the symmetry $G$ from (3.1) remains unbroken). In this case there is no Higgs-singlet mixing, but nevertheless the operator $\partial^{\mu}|\Phi|^{2} \partial_{\mu}|\Phi|^{2}$ is generated at 1-loop [60] with a Wilson coefficient $\bar{c}_{H}=\frac{n_{s} \lambda_{\mathrm{m}}^{2} v^{2}}{96 \pi^{2} m_{s}^{2}}$ (being $n_{s}$ the number of singlet scalar degrees of freedom). The universal suppresion of Higgs couplings can then be defined in a similar fashion to the previous case, $y^{2}=\bar{c}_{H}$.

We now discuss the current experimental constraints on the mass of the singlet-like state and the parameter $y$, coming from the latest measurements of Higgs boson signal strengths from ATLAS and CMS, from oblique corrections to EWPO and from direct searches of heavy Higgs scalars at LHC. For the analysis of Higgs signal strengths, we consider the up-to-date measurements by ATLAS and CMS in $h \rightarrow \gamma \gamma[61,62], h \rightarrow$ $Z Z^{*}[63,64], h \rightarrow W W^{*}[65,66], h \rightarrow \bar{b} b[67,68]$ and $h \rightarrow \tau \tau[69,70]$ final states, shown in table 2 . We then perform a combined $\chi^{2}$ fit

$$
\chi_{h}^{2}=\sum_{i}\left(\frac{\mu_{i}-\left(1-y^{2}\right)}{\Delta \mu_{i}}\right)^{2},
$$

with the various $\mu_{i} \equiv \mu_{\mathrm{obs}}^{i} / \mu_{\mathrm{SM}}^{i}$ and $\Delta \mu_{i}$ taken from table 2 , and with potential correlations among the different signal strength measurements not included in the fit. This yields an ATLAS and CMS combined limit $y<0.468$ at $95 \%$ C.L. via $\Delta \chi_{h}^{2}(y)=\chi_{h}^{2}(y)-\chi_{\min }^{2}=4$, as shown in figure 2 (horizontal solid-black). In addition, we obtain the 95\% C.L. exclusion prospects for $\mathrm{LHC}$ at $14 \mathrm{TeV}$ with $\mathcal{L}=300 \mathrm{fb}^{-1}$ (horizontal dotted-black) and $\mathcal{L}=3000 \mathrm{fb}^{-1}$ (HL-LHC, horizontal dashed-black). In doing so, we assume that future measurements of Higgs signal strengths will yield $\mu_{i}=1$, and use the projected 


\begin{tabular}{|c|c|}
\hline & $\mu_{\mathrm{obs}} / \mu_{\mathrm{SM}}$ \\
\hline ATLAS $\gamma \gamma 7+8 \mathrm{TeV}: \mu_{\mathrm{ggF}}$ & $1.32 \pm 0.38$ \\
\hline ATLAS $\gamma \gamma 7+8 \mathrm{TeV}: \mu_{\mathrm{VBF}}$ & $0.8 \pm 0.7$ \\
\hline ATLAS $W W^{*} 7+8 \mathrm{TeV}: \mu_{\mathrm{ggF}}$ & $0.82 \pm 0.36$ \\
\hline ATLAS $W W^{*} 7+8 \mathrm{TeV}: \mu_{\mathrm{VBF}}$ & $1.66 \pm 0.79$ \\
\hline ATLAS $Z Z^{*} 7+8 \mathrm{TeV}$ (inclusive) & $1.44_{-0.33}^{+0.40}$ \\
\hline ATLAS $b \bar{b} 7+8 \mathrm{TeV}: \mu_{\mathrm{VH}}$ & $0.2_{-0.6}^{+0.7}$ \\
\hline ATLAS $\tau \tau 7+8 \mathrm{TeV}: \mu_{\mathrm{ggF}}$ & $1.2_{-0.6}^{+0.8}$ \\
\hline ATLAS $\tau \tau 7+8 \mathrm{TeV}: \mu_{\mathrm{VBF}}$ & $1.6_{-0.5}^{+0.6}$ \\
\hline $\mathrm{CMS} \gamma \gamma$ (Mass Fit) $7+8 \mathrm{TeV}: \mu_{\mathrm{ggF}+\mathrm{ttH}}$ & $1.13_{-0.31}^{+0.37}$ \\
\hline CMS $\gamma \gamma$ (Mass Fit) $7+8 \mathrm{TeV}: \mu_{\mathrm{VBF}+\mathrm{VH}}$ & $1.16_{-0.58}^{+0.63}$ \\
\hline CMS $W W^{*} 7+8 \mathrm{TeV}(0 / 1$-jet $)$ & $0.74_{-0.20}^{+0.22}$ \\
\hline CMS $W W^{*} 7+8 \mathrm{TeV}$ (2-jets, VBF tag) & $0.60_{-0.46}^{+0.57}$ \\
\hline CMS $Z Z^{*} 7+8 \mathrm{TeV}$ (inclusive) & $0.93_{-0.24}^{+0.29}$ \\
\hline $\mathrm{CMS} b \bar{b} 7+8 \mathrm{TeV}: \mu_{\mathrm{VH}}$ & $1.0 \pm 0.5$ \\
\hline $\mathrm{CMS} \tau \tau 7+8 \mathrm{TeV}(0$-jet $)$ & $0.34 \pm 1.09$ \\
\hline $\mathrm{CMS} \tau \tau 7+8 \mathrm{TeV}$ (1-jet) & $1.07 \pm 0.46$ \\
\hline CMS $\tau \tau 7+8 \mathrm{TeV}$ (2-jets, VBF tag) & $0.94 \pm 0.41$ \\
\hline
\end{tabular}

Table 2. ATLAS and CMS measured Higgs Signal Strengths $\mu_{i} \equiv \mu_{\mathrm{obs}}^{i} / \mu_{\mathrm{SM}}^{i}$ in $h \rightarrow \gamma \gamma[61,62]$, $h \rightarrow Z Z^{*}[63,64], h \rightarrow W W^{*}[65,66], h \rightarrow \bar{b} b[67,68]$ and $h \rightarrow \tau \tau[69,70]$ final states.

CMS sensitivities ${ }^{4}[71] \Delta \mu_{300}^{\gamma \gamma}=0.06, \Delta \mu_{300}^{W W}=0.06, \Delta \mu_{300}^{Z Z}=0.07, \Delta \mu_{300}^{\tau \tau}=0.08$, $\Delta \mu_{300}^{b b}=0.11, \Delta \mu_{300}^{\mu \mu}=0.40, \Delta \mu_{3000}^{\gamma \gamma}=0.04, \Delta \mu_{3000}^{W W}=0.04, \Delta \mu_{3000}^{Z Z}=0.04, \Delta \mu_{3000}^{\tau \tau}=0.05$, $\Delta \mu_{3000}^{b b}=0.05, \Delta \mu_{3000}^{\mu \mu}=0.20$.

Turning to EWPO, we perform a fit to the oblique parameters $S, T, U$ using the best-fit values and standard deviations from the global analysis of the GFitter Group [72], with a SM reference point with $m_{t}=173 \mathrm{GeV}$ and a $126 \mathrm{GeV}$ Higgs mass. Under the assumption $U=0$, this yields

$$
\begin{aligned}
& \Delta S \equiv S-S_{\mathrm{SM}}=0.06 \pm 0.09 \\
& \Delta T \equiv T-T_{\mathrm{SM}}=0.10 \pm 0.07
\end{aligned} \quad \rho_{i j}=\left(\begin{array}{cc}
1 & 0.91 \\
0.91 & 1
\end{array}\right)
$$

being $\rho_{i j}$ the covariance matrix in the $S-T$ plane. The BSM corrections to $S$ and $T$ in the case of singlet-doublet mixing are given by

$$
\Delta S=\frac{1}{\pi} y^{2}\left[-H_{S}\left(\frac{m_{h}^{2}}{m_{Z}^{2}}\right)+H_{S}\left(\frac{m_{s}^{2}}{m_{Z}^{2}}\right)\right]
$$

\footnotetext{
${ }^{4}$ These assume that theoretical uncertainties improve by a factor $1 / 2$ compared to their present values, while all other systematic uncertainties improve by a factor $1 / \sqrt{\mathcal{L}}$.
} 


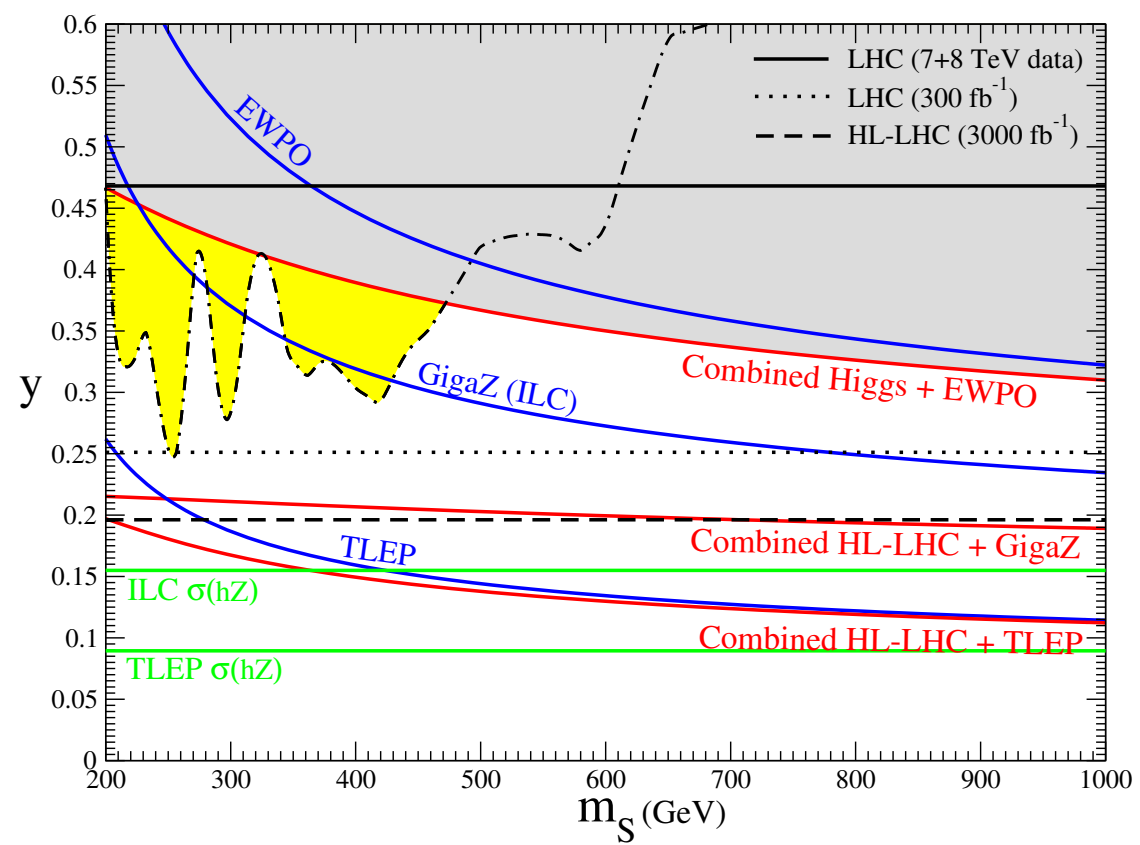

Figure 2. Present 95\% C.L. exclusion limits in the $\left(m_{s}, y\right)$ plane arising from ATLAS and CMS measurements of Higgs signal strengths as shown in table 2 (horizontal solid-black) and from EWPO (blue). The shaded grey region is excluded at 95\% C.L. by the combination (EWPO and Higgs signal strengths). The shaded yellow region may also be excluded by constraints from heavy scalar searches at LHC (dashed-dotted black), but these limits can be evaded in the presence of non-SM decays (see text). Also shown is the projected exclusion reach from Higgs signal strengths at the $14 \mathrm{TeV}$ run of LHC with $\mathcal{L}=300 \mathrm{fb}^{-1}$ (horizontal dotted-black) and at HL-LHC with $\mathcal{L}=3000 \mathrm{fb}^{-1}$ (horizontal dashed-black), from measurements of the $S$ and $T$ oblique parameters with ILC-GigaZ and TLEP (blue) and their combination with the HL-LHC exclusion reach (red), and from a precision measurement of the Higgs production cross section in association with a $Z$ boson $\sigma(h Z)$ (horizontal solid-green lines) at ILC $\left(\sqrt{s}=250 \mathrm{GeV}, \mathcal{L}=1150 \mathrm{fb}^{-1}\right)$ and $\operatorname{TLEP}\left(\sqrt{s}=240 \mathrm{GeV}, \mathcal{L}=500 \mathrm{fb}^{-1}\right)$.

$$
\Delta T=\frac{g^{2}}{16 \pi^{2} c_{W}^{2} \alpha_{\mathrm{EM}}} y^{2}\left[-H_{T}\left(\frac{m_{h}^{2}}{m_{Z}^{2}}\right)+H_{T}\left(\frac{m_{s}^{2}}{m_{Z}^{2}}\right)\right]
$$

with the functions $H_{S}(x)$ and $H_{T}(x)$ defined in appendix $\mathrm{C}$ of [73]. We then define

$$
\Delta \chi_{E W}^{2}\left(m_{s}, y\right)=\sum_{i, j}\left(\Delta \mathcal{O}_{i}\left(m_{s}, y\right)-\Delta \mathcal{O}_{i}^{0}\right)\left(\sigma^{2}\right)_{i j}^{-1}\left(\Delta \mathcal{O}_{j}\left(m_{s}, y\right)-\Delta \mathcal{O}_{j}^{0}\right)
$$

where $\Delta \mathcal{O}_{i}^{0}$ denote the central values in (3.17) and $\left(\sigma^{2}\right)_{i j} \equiv \sigma_{i} \rho_{i j} \sigma_{j}$, being $\sigma_{i}$ the $S$ and $T$ standard deviation from (3.17). We show in figure 2 the $95 \%$ C.L. exclusion limit $y\left(m_{s}\right)$ from $\Delta \chi_{E W}^{2}\left(m_{s}, y\right)$ (blue), together with the $95 \%$ C.L. exclusion limit $y\left(m_{s}\right)$ from the combination of $\Delta \chi_{h}^{2}(y)$ and $\Delta \chi_{E W}^{2}\left(m_{s}, y\right)$ (red). We also study the future exclusion reach that can be derived from prospects of measurements of EW precision observables by planned $e^{+} e^{-}$colliders (see e.g. [74]): assuming a SM best-fit value, the ILC GigaZ program's expected precision is $\sigma_{S}=0.017$ and $\sigma_{T}=0.022$ [72], while measurement of EWPO at TLEP could yield $\sigma_{S}=0.007$ and $\sigma_{T}=0.004[75,76]$. Figure 2 includes the $95 \%$ C.L. exclusion reach for $y\left(m_{s}\right)$ both for ILC and TLEP (blue), as well as the respective $95 \%$ C.L. exclusion 

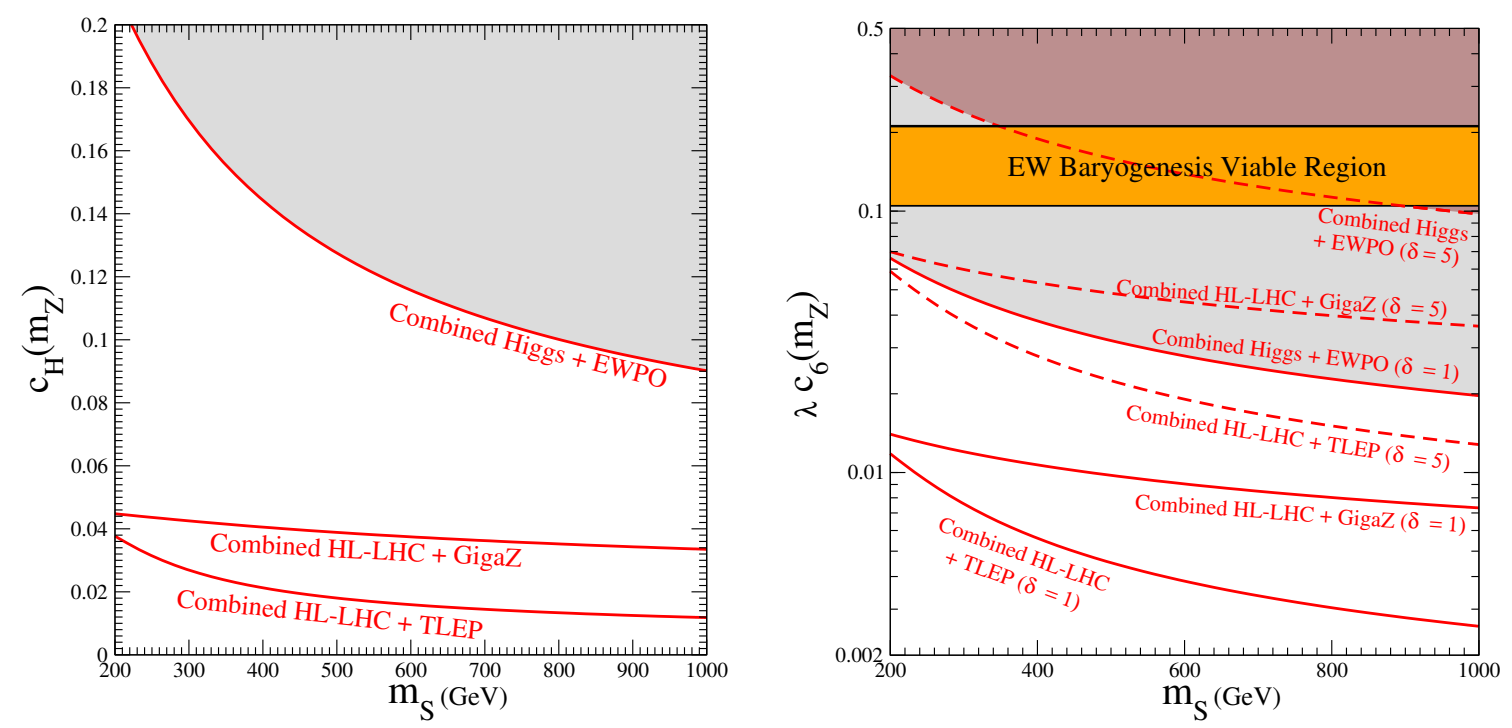

Figure 3. Present and future (as defined in figure 2) 95\% C.L. exclusion limits on the size of $\bar{c}_{H}\left(m_{Z}\right)$ (Left) and $\lambda \bar{c}_{6}\left(m_{Z}\right)$ (Right) as a function of $m_{s}$, from the combination of Higgs signal strengths and EW oblique parameters. For the case of $\lambda \bar{c}_{6}\left(m_{Z}\right)$, the region leading to a strongly first order EW phase transition as required by EW baryogenesis is shown as a horizontal orange band, while the grey (brown) region is currently excluded at 95\% C.L. for $\delta \equiv \mu_{m} / v_{s}=1(\delta=5)$.

reach when combined with HL-LHC $\left(\mathcal{L}=3000 \mathrm{fb}^{-1}\right)$. Figure 2 also shows the potential $95 \%$ C.L. exclusion reach from a precise measurement of the Higgs production cross section in association with a $Z$ boson $\sigma(h Z)$ at ILC with $\sqrt{s}=250 \mathrm{GeV}$ and $\mathcal{L}=1150 \mathrm{fb}^{-1}$ (with $\Delta \sigma(h Z) / \sigma(h Z) \sim 0.012$ ) [77] and at TLEP with $\sqrt{s}=240 \mathrm{GeV}$ and $\mathcal{L}=500 \mathrm{fb}^{-1}$ (with $\Delta \sigma(h Z) / \sigma(h Z) \sim 0.004)[75]$. From figure 2 it is evident that this precision measurement is potentially the most powerful probe of a non-SM Higgs admixture, significantly surpassing the reach of EWPO measurements.

It is worth stressing that the contributions to the $S$ and $T$ oblique parameters in the Higgs portal scenario could be re-derived in the EFT approach via operator mixing under renormalization group evolution (RGE), as shown e.g. in [78]. Specifically, the running of $\bar{c}_{H}$ from the matching scale $m_{s}$ down to $m_{Z}$ generates a contribution to $S$ and $T$, given at leading order by $[78,79]$

$$
\Delta S=\frac{1}{6 \pi} \bar{c}_{H}\left(m_{s}\right) \log \left(\frac{m_{s}}{m_{Z}}\right) \quad \Delta T=-\frac{3}{8 \pi c_{W}^{2}} \bar{c}_{H}\left(m_{s}\right) \log \left(\frac{m_{s}}{m_{Z}}\right)
$$

which correspond to the leading order contributions in (3.18) for $m_{s} \gg m_{Z}$.

Finally, figure 2 includes the latest 95\% C.L. limits on $y\left(m_{s}\right)$ (dotted-dashed black line and yellow region) obtained from CMS searches of a heavy neutral scalar decaying to $Z Z \rightarrow 4 \ell, Z Z \rightarrow 2 \ell 2 j$ and $Z Z \rightarrow 2 \ell 2 \nu$ final states [80] (searches in decays to $W W \rightarrow 2 \ell 2 \nu$ final states [81] are found not to be as sensitive). These constitute at present the most stringent constraint on $y$ for $m_{s} \lesssim 500 \mathrm{GeV}$, but we stress that for $m_{s}>2 m_{h}=250 \mathrm{GeV}$ these limits may be weakened/avoided for a significant branching fraction $\operatorname{Br}\left(h_{2} \rightarrow h_{1} h_{1}\right)$. 
From the present limits and future prospects discussed above, we can derive current/projected bounds on the size of the Wilson coefficients $\bar{c}_{H}\left(m_{Z}\right)$ and $\bar{c}_{6}\left(m_{Z}\right)$ in this scenario. From $\bar{c}_{H}\left(m_{s}\right)=y^{2}$ and $\lambda \bar{c}_{6}\left(m_{s}\right)=3 y^{2} \mu_{m} / v_{s}$, we perform a 1-loop RGE [28] (see also [29-31])

$$
\begin{aligned}
\bar{c}_{H}\left(m_{s}\right)-\bar{c}_{H}\left(m_{Z}\right) \simeq & \frac{\left[-\frac{9}{2} g^{2}-3 g^{\prime 2}+24 \lambda+12 y_{t}^{2}\right]}{16 \pi^{2}} \bar{c}_{H}\left(m_{s}\right) \log \left(\frac{m_{s}}{m_{Z}}\right) \\
\lambda \bar{c}_{6}\left(m_{s}\right)-\lambda \bar{c}_{6}\left(m_{Z}\right) \simeq & \frac{\left[-\frac{27}{2} g^{2}-\frac{9}{2} g^{\prime 2}+108 \lambda+18 y_{t}^{2}\right]}{16 \pi^{2}} \lambda \bar{c}_{6}\left(m_{s}\right) \log \left(\frac{m_{s}}{m_{Z}}\right) \\
& +\frac{\left[-3 g^{2}+40 \lambda\right]}{8 \pi^{2}} \lambda \bar{c}_{H}\left(m_{s}\right) \log \left(\frac{m_{s}}{m_{Z}}\right)
\end{aligned}
$$

Current bounds on $\bar{c}_{H}\left(m_{Z}\right)$ and $\bar{c}_{6}\left(m_{Z}\right)$ as a function of the new physics scale $m_{s}$, as well as the projected $95 \%$ C.L. exclusion sensitivity from the combination of HL-LHC and GigaZ, and HL-LHC and TLEP EWPO (TeraZ) measurements, are shown in figure 3.

As recently noted in [78], a sizeable $\bar{c}_{6}\left(m_{Z}\right)$ could in this scenario lead to a strongly first order EW phase transition, potentially allowing for EW baryogenesis in the Early Universe [82-84]. Requiring a sufficiently strong first order phase transition imposes a lower bound $\lambda \bar{c}_{6}\left(m_{Z}\right) \gtrsim 0.105,{ }^{5}$ while the successful completion of the phase transition sets the upper bound $\lambda \bar{c}_{6}\left(m_{Z}\right) \lesssim 0.211$ [83]. As shown in figure 3 (Right), the present combination of measured Higgs signal strengths and EW precision data rules out a strong EW phase transition, except for very large values $\delta \equiv \mu_{m} / v_{s} \gg 1$ combined with relatively low singlet masses $m_{s}$. These results are somewhat stronger than those of [78], the reason being that the analysis of the EW phase transition in the presence of $\bar{c}_{6}$ from [82] (with reference [78] follows) considers thermal masses as the only thermal effects in the effective potential, and tends to overestimate the strength of the EW phase transition as compared to a full 1-loop analysis [83]. Finally, while we stress that our results certainly do not rule out a strong EW phase transition in the singlet scalar extension of the SM (see e.g. [47]), they imply that having it originate from the presence of $\bar{c}_{6}$ is currently challenging, and the entire region of parameter space will be covered by the combination of HL-LHC and GigaZ.

Let us finish this section on the extension of the SM Higgs sector via a singlet scalar by summarizing the results. We have seen that the EFT leads to just two non-zero operators at tree-level,

$$
\begin{aligned}
\bar{c}_{H} & =y^{2} \text { (mixing) } \\
\bar{c}_{H} & =\frac{n_{s}}{96 \pi^{2}}\left(\frac{\lambda_{m} v}{m_{s}}\right)^{2} \text { (no mixing) } \\
\lambda \bar{c}_{6} & =3 \delta \bar{c}_{H} \text { (only w. explicit symmetry breaking) }
\end{aligned}
$$

\subsection{Two Higgs doublet models}

Theories with two scalar doublets appear in a wide variety of scenarios, ranging from the MSSM and its extensions [85, 86] to models of dark matter [87-89] and neutrino

\footnotetext{
${ }^{5}$ The EW phase transition occurs at $T=T_{c} \simeq 100 \mathrm{GeV}$, and we can approximate $T_{c} \simeq m_{Z}$ for $\bar{c}_{6}$.
} 


\begin{tabular}{|c|c|c|c|}
\hline & Up-type Quarks & Down-type Quarks & Charged Leptons \\
\hline Type - I & $\Phi_{2}$ & $\Phi_{2}$ & $\Phi_{2}$ \\
\hline Type - II & $\Phi_{2}$ & $\Phi_{1}$ & $\Phi_{1}$ \\
\hline$\ell-$ Specific & $\Phi_{2}$ & $\Phi_{2}$ & $\Phi_{1}$ \\
\hline Flipped & $\Phi_{2}$ & $\Phi_{1}$ & $\Phi_{2}$ \\
\hline
\end{tabular}

Table 3. Classification of $2 \mathrm{HDM}$ with a $\mathbb{Z}_{2}$-symmetry in the Yukawa sector. For each 2HDM-type, we indicate which scalar doublet couples to up-type quarks, down-type quarks and charged leptons.

masses (see e.g. [90-92]). The phenomenology of Two Higgs Doublet Model (2HDM) scenarios has been widely studied in the literature (for a general review of $2 \mathrm{HDMs}$, see [93]), including the impact of measured properties of the observed Higgs boson on the 2HDM parameter space [94-99, 101, 102], the possible connection to the EW phase transition and baryogenesis [103-106] and its potential LHC signatures [107-113].

We consider here $2 \mathrm{HDM}$ scenarios with a $\mathbb{Z}_{2}$-symmetry which is at most softly broken, avoiding tree-level Flavor Changing Neutral Currents (FCNCs) in the Yukawa sector [114]. This $\mathbb{Z}_{2}$-symmetry leads to four types of $2 \mathrm{HDM}$ (see e.g. [93] for details), according to the way in which $\Phi_{1,2}$ are coupled to the different SM fermion species, as shown in table 3 .

The scalar potential for the two scalar doublets $\Phi_{1,2}$ reads

$$
\begin{aligned}
V_{\text {tree }}\left(\Phi_{1}, \Phi_{2}\right)= & \mu_{1}^{2}\left|\Phi_{1}\right|^{2}+\mu_{2}^{2}\left|\Phi_{2}\right|^{2}-\left[\mu^{2} \Phi_{1}^{\dagger} \Phi_{2}+\text { h.c. }\right]+\frac{\lambda_{1}}{2}\left|\Phi_{1}\right|^{4} \\
& +\frac{\lambda_{2}}{2}\left|\Phi_{2}\right|^{4}+\lambda_{3}\left|\Phi_{1}\right|^{2}\left|\Phi_{2}\right|^{2}+\lambda_{4}\left|\Phi_{1}^{\dagger} \Phi_{2}\right|^{2}+\frac{1}{2}\left[\lambda_{5}\left(\Phi_{1}^{\dagger} \Phi_{2}\right)^{2}+\text { h.c. }\right] .
\end{aligned}
$$

In the following, we consider CP-conserving scenarios and set $\lambda_{5}$ and $\mu^{2}$ to be real. The doublets and their vevs at the EW minimum can be written as

$$
\Phi_{k}=\left(\begin{array}{c}
\varphi_{k}^{+} \\
\frac{h_{k}+i \eta_{k}}{\sqrt{2}}
\end{array}\right), \quad\left\langle\Phi_{1}\right\rangle=\left(\begin{array}{c}
0 \\
\frac{v_{1}}{\sqrt{2}}
\end{array}\right), \quad\left\langle\Phi_{2}\right\rangle=\left(\begin{array}{c}
0 \\
\frac{v_{2}}{\sqrt{2}}
\end{array}\right),
$$

with $v_{1}^{2}+v_{2}^{2}=v=246 \mathrm{GeV}$ and $v_{2} / v_{1}=\tan \beta$. The angle $\beta$ also parametrizes the rotation to the mass eigenbasis for the charged states $G^{ \pm}, H^{ \pm}$and neutral CP-odd $G^{0}, A^{0}$ states, with $G^{ \pm}, G^{0}$ being the Goldstone bosons and $H^{ \pm}, A^{0}$ the physical states. We likewise define $\alpha$ to be the mixing angle parametrizing the rotation to the mass eigenbasis for the CP-even neutral states $h, H^{0}$ (see appendix A.1). The $\mu$ parameter is responsible for the soft-breaking of the $\mathbb{Z}_{2}$-symmetry in (3.25). After EW symmetry breaking, the parameters $\mu_{i}, \lambda_{i}$ in (3.25) may be written in terms of the masses of the physical states $m_{h}, m_{H^{0}}, m_{A^{0}}$, $m_{H^{ \pm}}$, the mixing angles $\alpha, \beta$ and the $\mu$ parameter, as shown in appendix A.1.

In order to obtain an EFT for the SM Higgs, we may perform an SU(2) rotation from the field basis $\Phi_{1}, \Phi_{2}$ to a basis $H_{1}, H_{2}$ in which $\left\langle H_{1}\right\rangle=\frac{v}{\sqrt{2}}$ and $\left\langle H_{2}\right\rangle=0$ (the socalled Higgs basis). This rotation is precisely parametrized by the angle $\beta$. After the field 
rotation, the scalar potential for $H_{1,2}$ reads

$$
\begin{aligned}
V_{\text {tree }}\left(H_{1}, H_{2}\right)= & \tilde{\mu}_{1}^{2}\left|H_{1}\right|^{2}+\tilde{\mu}_{2}^{2}\left|H_{2}\right|^{2}-\tilde{\mu}^{2}\left[H_{1}^{\dagger} H_{2}+\text { H.c. }\right]+\frac{\tilde{\lambda}_{1}}{2}\left|H_{1}\right|^{4} \\
& +\frac{\tilde{\lambda}_{2}}{2}\left|H_{2}\right|^{4}+\tilde{\lambda}_{3}\left|H_{1}\right|^{2}\left|H_{2}\right|^{2}+\tilde{\lambda}_{4}\left|H_{1}^{\dagger} H_{2}\right|^{2}+\frac{\tilde{\lambda}_{5}}{2}\left[\left(H_{1}^{\dagger} H_{2}\right)^{2}+\text { H.c. }\right] \\
& +\tilde{\lambda}_{6}\left[\left|H_{1}\right|^{2} H_{1}^{\dagger} H_{2}+\text { H.c. }\right]+\tilde{\lambda}_{7}\left[\left|H_{2}\right|^{2} H_{1}^{\dagger} H_{2}+\text { H.c. }\right]
\end{aligned}
$$

with $\tilde{\mu}_{i}, \tilde{\mu}$ and $\tilde{\lambda}_{i}$ being functions of the original parameters in (3.25). We note that the field rotation may generate $\tilde{\lambda}_{6,7}$ even if initially absent from (3.25) due to the $\mathbb{Z}_{2}$-symmetry.

We may then construct an effective Lagrangian for $H_{1}$ by matching to the theory with the second doublet $H_{2}$ at the scale $\tilde{\mu}^{2}$, assuming $\tilde{\mu}_{2}^{2} \gg v^{2}$. We stress however that the doublet $H_{1}$ can only be fully identified with the SM Higgs doublet in the alignment limit $\cos (\beta-\alpha) \equiv c_{\beta-\alpha} \rightarrow 0$, where mixing in the CP-even sector is absent (see the discussion in appendix A.1). Away from alignment $(\alpha \neq \beta-\pi / 2)$, these mixing effects lead to tree-level modifications of SM Higgs couplings (see e.g. the discussion in [115]). For a general 2HDM, $c_{\beta-\alpha}=0$ is possible at tree-level ${ }^{6}[116,117]$, and indeed the latest analyses of Higgs data by ATLAS and CMS strongly prefer $c_{\beta-\alpha}^{2} \ll 1$, as shown in section 3.2.3.

In the following, we introduce and discuss two benchmark scenarios for the use of Higgs Effective Theory in 2HDM: an exact alignment scenario $c_{\beta-\alpha}=0$ and a scenario with $\left|c_{\beta-\alpha}\right| \ll 1$ (MSSM-like scenario). We then analyze the constraints on the $2 \mathrm{HDM}$ parameter space obtained from measurement of Higgs signal strengths and EW Oblique Parameters, and the corresponding constraint on the values of the Wilson coefficients of the Higgs EFT.

\subsubsection{Benchmark A: exact alignment $c_{\beta-\alpha}=0$}

As discussed above, in this scenario mixing effects are absent and $H_{1}$ is precisely the SM Higgs doublet $\Phi$. The scalar potential (3.27) simplifies in this limit, since $\tilde{\lambda}_{6}$ and $\tilde{\mu}$ are both $\propto c_{\beta-\alpha}$ and vanish as shown in (A.16)-(A.17). We then match the $2 \mathrm{HDM}$ to an $\mathrm{SU}(2)_{L} \times$ $\mathrm{U}(1)_{Y}$ invariant EFT with the field content of the SM, being $H_{2}$ the only massive field which will be decoupled in the matching calculation. The $D=6$ effective operators from (2.1) are generated first at 1-loop order, with the corresponding Wilson coefficients given by

$$
\begin{aligned}
\bar{c}_{H} & =-\left[-4 \tilde{\lambda}_{3} \tilde{\lambda}_{4}+\tilde{\lambda}_{4}^{2}+\tilde{\lambda}_{5}^{2}-4 \tilde{\lambda}_{3}^{2}\right] \frac{v^{2}}{192 \pi^{2} \tilde{\mu}_{2}^{2}} \\
\bar{c}_{6} & =-\left(\tilde{\lambda}_{4}^{2}+\tilde{\lambda}_{5}^{2}\right) \frac{v^{2}}{192 \pi^{2} \tilde{\mu}_{2}^{2}} \\
\bar{c}_{T} & =\left(\tilde{\lambda}_{4}^{2}-\tilde{\lambda}_{5}^{2}\right) \frac{v^{2}}{192 \pi^{2} \tilde{\mu}_{2}^{2}} \\
\bar{c}_{\gamma} & =\frac{m_{W}^{2} \tilde{\lambda}_{3}}{256 \pi^{2} \tilde{\mu}_{2}^{2}}
\end{aligned}
$$

\footnotetext{
${ }^{6}$ In contrast, for the MSSM $c_{\beta-\alpha} \rightarrow 0$ is only obtained at tree-level in the decoupling limit $\tilde{\mu}_{2}^{2} \rightarrow \infty$. Moreover, $c_{\beta-\alpha}=0$ at loop level is only possible in a very small portion of parameter space [116, 117].
} 


$$
\begin{aligned}
\bar{c}_{W} & =-\bar{c}_{H W}=\frac{m_{W}^{2}\left(2 \tilde{\lambda}_{3}+\tilde{\lambda}_{4}\right)}{192 \pi^{2} \tilde{\mu}_{2}^{2}}=\frac{8}{3} \bar{c}_{\gamma}+\frac{m_{W}^{2} \tilde{\lambda}_{4}}{192 \pi^{2} \tilde{\mu}_{2}^{2}} \\
\bar{c}_{B} & =-\bar{c}_{H B}=\frac{m_{W}^{2}\left(-2 \tilde{\lambda}_{3}+\tilde{\lambda}_{4}\right)}{192 \pi^{2} \tilde{\mu}_{2}^{2}}=-\frac{8}{3} \bar{c}_{\gamma}+\frac{m_{W}^{2} \tilde{\lambda}_{4}}{192 \pi^{2} \tilde{\mu}_{2}^{2}} \\
\bar{c}_{3 W} & =\frac{\bar{c}_{2 W}}{3}=\frac{m_{W}^{2}}{1440 \pi^{2} \tilde{\mu}_{2}^{2}}
\end{aligned}
$$

from which we immediately obtain

$$
\bar{c}_{W}+\bar{c}_{B}=\frac{m_{W}^{2} \tilde{\lambda}_{4}}{96 \pi^{2} \tilde{\mu}_{2}^{2}} \quad, \quad \bar{c}_{W}-\bar{c}_{B}=\frac{16}{3} \bar{c}_{\gamma}=\frac{m_{W}^{2} \tilde{\lambda}_{3}}{48 \pi^{2} \tilde{\mu}_{2}^{2}}
$$

Before we continue, let us note that $\bar{c}_{\gamma}, \bar{c}_{W}$ and $\bar{c}_{B}$ may take positive and negative values, as the bounded-from-below conditions (A.2) do not restrict either possibility. The various relations among the Wilson coefficients $\bar{c}_{H W}, \bar{c}_{W}, \bar{c}_{H B}, \bar{c}_{B}$ and $\bar{c}_{\gamma}$ in (3.28) imply that the $D=6$ effective operators $\mathcal{O}_{H W}, \mathcal{O}_{W}, \mathcal{O}_{H B}, \mathcal{O}_{B}$ and $\mathcal{O}_{\gamma}$ can in fact be re-casted in terms of three operators

$$
\frac{g^{2} \bar{c}_{W W}}{m_{W}^{2}}\left|H_{1}\right|^{2} W_{\mu \nu}^{k} W_{k}^{\mu \nu}+\frac{2 g g^{\prime} \bar{c}_{W B}}{m_{W}^{2}}\left[H_{1}^{\dagger} T_{2 k} H_{1}\right] W_{\mu \nu}^{k} B^{\mu \nu}+\bar{c}_{\gamma} \mathcal{O}_{\gamma}
$$

with

$$
\begin{aligned}
\frac{g^{2}}{m_{W}^{2}}\left|H_{1}\right|^{2} W_{\mu \nu}^{k} W_{k}^{\mu \nu} & \equiv \mathcal{O}_{W W}=4\left(\mathcal{O}_{W}-\mathcal{O}_{B}+\mathcal{O}_{H B}-\mathcal{O}_{H W}\right)+\mathcal{O}_{\gamma} \\
\frac{2 g g^{\prime}}{m_{W}^{2}}\left[H_{1}^{\dagger} T_{2 k} H_{1}\right] W_{\mu \nu}^{k} B^{\mu \nu} & \equiv \mathcal{O}_{W B}=4\left(\mathcal{O}_{B}-\mathcal{O}_{H B}\right)-\mathcal{O}_{\gamma}
\end{aligned}
$$

The three operators in (3.30) share the common feature that they do not involve derivatives of the Higgs field $H_{1}$. This very property is in fact responsible for the relations among Wilson coefficients in (3.28), since the Feynman diagrams involved in the EFT matching (see figure 4-left) do not involve Higgs field derivatives.

\subsubsection{Benchmark B: departure from alignment $c_{\beta-\alpha} \ll 1$ (MSSM-like)}

Upon departure from the alignment limit, mixing in the CP-even sector leads to several effects that are absent for $c_{\beta-\alpha}=0$ : first, there is a modification of the couplings of the Higgs boson $h$ to gauge bosons and fermions at tree-level, parametrized in terms of $\kappa$ factors for vector bosons $\kappa_{V} \equiv g_{h V_{\mu} V_{\nu}} / g_{h V_{\mu} V_{\nu}}^{\mathrm{SM}}$ and fermions $\kappa_{f} \equiv g_{h \bar{f} f} / g_{h \bar{f} f}^{\mathrm{SM}}$ (for up-type quarks $\kappa_{u}$, down-type quarks $\kappa_{d}$ and leptons $\kappa_{\ell}$ ). For the various Types of 2HDM from table 3 , these are given in terms of $c_{\beta-\alpha}$ and $t_{\beta} \equiv \tan \beta$ as

$$
\begin{gathered}
\text { Type }-\mathrm{I}: \kappa_{V}=s_{\beta-\alpha} ; \kappa_{u}=\kappa_{d}=\kappa_{\ell}=\frac{c_{\beta-\alpha}}{t_{\beta}}+s_{\beta-\alpha} \\
\text { Type }-\mathrm{II}: \kappa_{V}=s_{\beta-\alpha} ; \kappa_{u}=\frac{c_{\beta-\alpha}}{t_{\beta}}+s_{\beta-\alpha} ; \kappa_{d}=\kappa_{\ell}=s_{\beta-\alpha}-t_{\beta} c_{\beta-\alpha} \\
\ell-\text { Specific : } \kappa_{V}=s_{\beta-\alpha} ; \kappa_{u}=\kappa_{d}=\frac{c_{\beta-\alpha}}{t_{\beta}}+s_{\beta-\alpha} ; \kappa_{\ell}=s_{\beta-\alpha}-t_{\beta} c_{\beta-\alpha}
\end{gathered}
$$




$$
\text { Flipped : } \kappa_{V}=s_{\beta-\alpha} ; \kappa_{u}=\kappa_{\ell}=\frac{c_{\beta-\alpha}}{t_{\beta}}+s_{\beta-\alpha} ; \kappa_{d}=s_{\beta-\alpha}-t_{\beta} c_{\beta-\alpha}
$$

In addition, away from alignment $g_{H^{0} h h}$ (coupling between $H^{0}$ and two Higgs bosons $h$ ), $g_{H^{0} V_{\mu} V_{\nu}}$ (coupling between $H^{0}$ and two gauge bosons $V_{\mu}$ ) and $g_{\phi V_{\mu} h}$ (couplings among $h$, a gauge boson $V_{\mu}$ and $\left.\phi=A^{0}, H^{ \pm}\right)$are non-zero, giving rise to 1-loop diagrams contributing to the vertices $V_{\mu} V_{\nu} h$ and $V_{\mu} V_{\nu} V_{\rho}$ with both heavy $\left(H^{0}, A^{0}, H^{ \pm}\right)$and light $\left(h, V_{\mu}\right)$ states running in the loop.

Let us discuss all these effects in an $\mathrm{SU}(2)_{L} \times \mathrm{U}(1)_{Y}$ invariant Higgs EFT approach. We first note that $\left\langle H_{1}\right\rangle=\frac{v}{\sqrt{2}}$ and $\left\langle H_{2}\right\rangle=0$ in (3.27) imply the relations

$$
\tilde{\mu}_{1}^{2}=-\frac{\tilde{\lambda}_{1} v^{2}}{2} \quad, \quad \tilde{\mu}^{2}=\tilde{\lambda}_{6} v^{2}
$$

which may be simply regarded as minimization conditions. The mass matrix for the neutral $\mathrm{CP}$-even states is non-diagonal, brought into a diagonal form via the rotation matrix $U$

$$
U=\left(\begin{array}{cc}
1 & \epsilon \\
-\epsilon & 1
\end{array}\right) \quad \epsilon=-\frac{\tilde{\mu}^{2}}{2 \tilde{\mu}_{2}^{2}}+\frac{3}{2} \frac{\tilde{\lambda}_{6} v^{2}}{\tilde{\mu}_{2}^{2}}=\frac{\tilde{\lambda}_{6} v^{2}}{\tilde{\mu}_{2}^{2}}
$$

The deviation from alignment is then parametrized by $\left(\tilde{\lambda}_{6} v^{2}\right) / \tilde{\mu}_{2}^{2}$, which matches the corresponding expression for $c_{\beta-\alpha}$ after EW symmetry breaking (see appendix A.1)

$$
c_{\beta-\alpha}^{2} \sim \frac{\left(\tilde{\lambda}_{6} v^{2}\right)^{2}}{\tilde{\mu}_{2}^{4}} \sim \frac{v^{4}}{\tilde{\mu}_{2}^{4}} \ll 1,
$$

recovering the well-known scaling result for $c_{\beta-\alpha}^{2}$ in $2 \mathrm{HDM}$ s as the decoupling limit is approached [116].

At tree-level, the EFT matching generates effective operators suppressed at least by $1 / \tilde{\mu}_{2}^{4}$ (except for $\bar{c}_{6}$, which receives a further contribution suppressed only by $1 / \tilde{\mu}_{2}^{2}$ ), with operators of different $D$ contributing to order $v^{4} / \tilde{\mu}_{2}^{4}$ :

$$
\begin{aligned}
\mathcal{L}_{\text {Eff }}^{\text {tree }} \supset & \frac{\bar{c}_{2 h} \tilde{\mu}^{4}}{\tilde{\mu}_{2}^{4}}\left(D_{\mu} H_{1}^{\dagger}\right)\left(D^{\mu} H_{1}\right)+\frac{\bar{c}_{4 h} \tilde{\mu}^{2}}{\tilde{\mu}_{2}^{4}} \partial^{\mu}\left|H_{1}\right|^{2} \partial_{\mu}\left|H_{1}\right|^{2} \\
& +\frac{\bar{c}_{6 h}}{\tilde{\mu}_{2}^{4}} D_{\mu}\left(H_{1}^{\dagger}\left|H_{1}\right|^{2}\right) D^{\mu}\left(H_{1}\left|H_{1}\right|^{2}\right)-\left(\frac{\tilde{\lambda}_{6} \tilde{\mu}^{2}}{\tilde{\mu}_{2}^{4}}-\frac{\tilde{\lambda}_{6}^{2}}{\lambda \tilde{\mu}_{2}^{2}}\right)\left|H_{1}\right|^{6} .
\end{aligned}
$$

The matching procedure yields $\bar{c}_{6 h}=\tilde{\lambda}_{6}^{2}, \bar{c}_{4 h}=\bar{c}_{H}=0$ and $\bar{c}_{2 h}=1$, and use of the relation $\tilde{\mu}^{2}=\tilde{\lambda}_{6} v^{2}$ results in both the first and the third term in (3.40) contributing at order $\left(\tilde{\lambda}_{6} v^{2}\right)^{2} / \tilde{\mu}_{2}^{4}$. The first term rescales both the SM Higgs kinetic term and its couplings to gauge boson by the same amount, so it does not have a net effect. The third term does however include a rescaling of the SM Higgs kinetic term $\propto c_{\beta-\alpha}^{2}$ that is not compensated by a similar one in the gauge boson interactions, leading to the well-known tree-level deviation from the SM Higgs couplings to gauge bosons away from alignment, proportional to $c_{\beta-\alpha}^{2}$. 

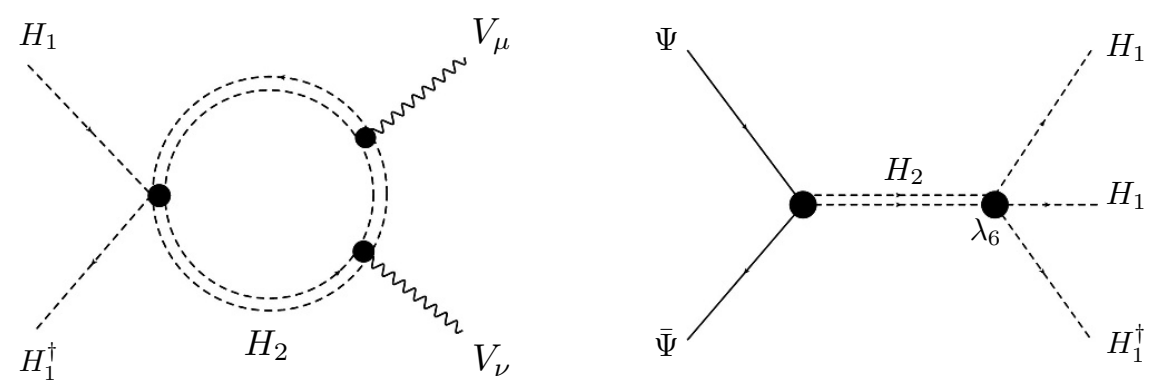

Figure 4. Left: Feynman diagram responsible for anomalous Higgs couplings to gauge boson at 1loop. Right: Feynman diagram responsible for anomalous Higgs couplings to fermions at tree-level.

Regarding $\bar{c}_{6}$, the presence of a non-zero $\tilde{\lambda}_{6}$ also yields an extra contribution at 1-loop as compared to (3.28). The full result for $\bar{c}_{6}$ away from alignment is

$$
\bar{c}_{6}=-\frac{\left(\tilde{\lambda}_{4}^{2}+\tilde{\lambda}_{5}^{2}+12 \tilde{\lambda}_{6}^{2}\right) v^{2}}{192 \pi^{2} \tilde{\mu}_{2}^{2}}-\left(\frac{\tilde{\lambda}_{6}^{2} v^{2}}{\lambda \tilde{\mu}_{2}^{2}}-\frac{\tilde{\lambda}_{6} \tilde{\mu}^{2} v^{2}}{\tilde{\mu}_{2}^{4}}\right)
$$

Let us also stress that similarly to the tree-level effects just discussed, the extra 1loop diagrams appearing away from alignment (involving both light and heavy degrees of freedom) are proportional to $c_{\beta-\alpha}^{2}$. Thus the contribution of these diagrams to the various Wilson coefficients from (2.2) and (2.5) is at least of order $\left(v^{4} / \tilde{\mu}_{2}^{4}\right) \times 1$-loop and can be safely neglected. As a result, the values of the Wilson coefficients $\bar{c}_{T}, \bar{c}_{H W}, \bar{c}_{W}, \bar{c}_{H B}, \bar{c}_{B}$, $\bar{c}_{\gamma}$ for $c_{\beta-\alpha}^{2} \ll 1$ remains unchanged w.r.t. the alignment scenario.

The interactions between the SM Higgs and fermions receive tree-level corrections of the form shown in figure 4-right away from the alignment limit, encoded in the $D=6$ effective operators

$$
\mathcal{L}_{\mathrm{SILH}}^{(6)}=\left[\frac{\bar{c}_{u} y_{u}}{v^{2}} H_{1}^{\dagger} H_{1} \bar{Q}_{L} H_{1}^{\dagger} u_{R}+\frac{\bar{c}_{d} y_{d}}{v^{2}} H_{1}^{\dagger} H_{1} \bar{Q}_{L} H_{1} d_{R}+\frac{\bar{c}_{\ell} y_{\ell}}{v^{2}} H_{1}^{\dagger} H_{1} \bar{L}_{L} H_{1} \ell_{R}\right],
$$

with

$$
\bar{c}_{u} y_{u}=\frac{m_{u} f_{u}}{v} \frac{\tilde{\lambda}_{6} v^{2}}{\tilde{\mu}_{2}^{2}} \quad \bar{c}_{d} y_{d}=\frac{m_{d} f_{d}}{v} \frac{\tilde{\lambda}_{6} v^{2}}{\tilde{\mu}_{2}^{2}} \quad \bar{c}_{\ell} y_{\ell}=\frac{m_{\ell} f_{\ell}}{v} \frac{\tilde{\lambda}_{6} v^{2}}{\tilde{\mu}_{2}^{2}}
$$

and $f_{u, d, \ell}$ depending of the 2HDM-Type under consideration, and given by

$$
\begin{array}{r}
\text { Type - I : } \quad f_{u}=f_{d}=f_{\ell}=t_{\beta}^{-1} \\
\text { Type- II : } f_{u}=t_{\beta}^{-1} ; f_{d}=f_{\ell}=-t_{\beta} \\
\ell-\text { Specific : } f_{u}=f_{d}=t_{\beta}^{-1} ; f_{\ell}=-t_{\beta} \\
\text { Flipped : } f_{u}=f_{\ell}=t_{\beta}^{-1} ; f_{d}=-t_{\beta}
\end{array}
$$

These results reproduce the $\kappa$-factors from (3.33)-(3.36) to leading order in $c_{\beta-\alpha}$.

\subsubsection{HDM constraints: Higgs signal strengths \& EW oblique parameters}

The ATLAS and CMS measurements of Higgs boson signal strengths from table 2 constrain the allowed parameter region of the $2 \mathrm{HDM}$ in the plane $\left(c_{\beta-\alpha}, \tan \beta\right)$ (see e.g. the analyses 


\begin{tabular}{|l|c|}
\hline & $\bar{\mu} \equiv \mu(\kappa) / \mu_{\mathrm{SM}}$ \\
\hline$\gamma \gamma: \mu_{\mathrm{ggF}}$ & $\kappa_{u}^{2} \times \kappa_{\gamma}\left(\kappa_{u}, \kappa_{V}\right)^{2} \times \Gamma\left(\kappa_{d}, \kappa_{V}, \kappa_{u}, \kappa_{\ell}\right)^{-1}$ \\
$\gamma \gamma: \mu_{\mathrm{VBF}}$ & $\kappa_{V}^{2} \times \kappa_{\gamma}\left(\kappa_{u}, \kappa_{V}\right)^{2} \times \Gamma\left(\kappa_{d}, \kappa_{V}, \kappa_{u}, \kappa_{\ell}\right)^{-1}$ \\
$W W^{*}: \mu_{\mathrm{ggF}}-0 / 1$-jet & $\kappa_{u}^{2} \times \kappa_{V}^{2} \times \Gamma\left(\kappa_{d}, \kappa_{V}, \kappa_{u}, \kappa_{\ell}\right)^{-1}$ \\
$W W^{*}: \mu_{\mathrm{VBF}}$ & $\kappa_{V}^{4} \times \Gamma\left(\kappa_{d}, \kappa_{V}, \kappa_{u}, \kappa_{\ell}\right)^{-1}$ \\
$Z Z^{*}:$ inclusive & $\kappa_{u}^{2} \times \kappa_{V}^{2} \times \Gamma\left(\kappa_{d}, \kappa_{V}, \kappa_{u}, \kappa_{\ell}\right)^{-1}$ \\
$b \bar{b}: \mu_{\mathrm{VH}}$ & $\kappa_{V}^{2} \times \kappa_{d}^{2} \times \Gamma\left(\kappa_{d}, \kappa_{V}, \kappa_{u}, \kappa_{\ell}\right)^{-1}$ \\
$\tau \tau: \mu_{\mathrm{ggF}-0 / 1-j e t}$ & $\kappa_{u}^{2} \times \kappa_{\ell}^{2} \times \Gamma\left(\kappa_{d}, \kappa_{V}, \kappa_{u}, \kappa_{\ell}\right)^{-1}$ \\
$\tau \tau: \mu_{\mathrm{VBF}}$ & $\kappa_{V}^{2} \times \kappa_{\ell}^{2} \times \Gamma\left(\kappa_{d}, \kappa_{V}, \kappa_{u}, \kappa_{\ell}\right)^{-1}$ \\
\hline
\end{tabular}

Table 4. Modified (by $\kappa$-factors) Higgs Signal Strengths $\bar{\mu}_{i}$.

from $[94,96-98,101,108])$ through a combined $\chi^{2}$ fit, which in this case treats the different signal strength measurements as independent (not correlated)

$$
\chi_{h}^{2}\left(c_{\beta-\alpha}, t_{\beta}\right)=\sum_{i}\left(\frac{\mu_{i}-\bar{\mu}_{i}\left(c_{\beta-\alpha}, t_{\beta}\right)}{\Delta \mu_{i}}\right)^{2} .
$$

Each $\bar{\mu}_{i}$ (the expected signal strength for each channel) may be expressed in terms of rescaling $\kappa$-factors for the coupling of the Higgs boson to vector bosons $\kappa_{V}$, up-type quarks $\kappa_{u}$, down-type quarks $\kappa_{d}$ and leptons $\kappa_{\ell}$. The expression of each $\bar{\mu}_{i}$ corresponding to each process considered in table 2 in terms of these $\kappa$-factors is given in table 4 with $\kappa_{i}$ defined in (3.33)-(3.36) and $\kappa_{\gamma}\left(\kappa_{u}, \kappa_{V}\right)=1.26 \kappa_{V}-0.26 \kappa_{u}$, and $\Gamma\left(\kappa_{d}, \kappa_{V}, \kappa_{u}, \kappa_{\ell}\right)$ given by

$$
\Gamma\left(\kappa_{d}, \kappa_{V}, \kappa_{u}, \kappa_{\ell}\right)=0.2427 \kappa_{V}^{2}+0.1124 \kappa_{u}^{2}+0.578 \kappa_{d}^{2}+0.0637 \kappa_{\ell}^{2}
$$

We present the $68 \%, 95 \%$ and $99 \%$ C.L. limits on the $\left(c_{\beta-\alpha}, t_{\beta}\right)$ plane from global fits of the light Higgs boson couplings, given respectively by $\Delta \chi_{h}^{2}\left(c_{\beta-\alpha}, t_{\beta}\right) \equiv \chi_{h}^{2}\left(c_{\beta-\alpha}, t_{\beta}\right)-$ $\chi_{\min }^{2}=2.27, \Delta \chi_{h}^{2}\left(c_{\beta-\alpha}, t_{\beta}\right)=5.99$ and $\Delta \chi_{h}^{2}\left(c_{\beta-\alpha}, t_{\beta}\right)=9.21$, for the different Types of $2 \mathrm{HDM}$ in figure 5 . These are particularly stringent for Type II and Flipped as compared to Lepton-Specific and specially to Type I, due to the different nature of the Higgs coupling to down-type quarks. Figure 5 also includes the projected $95 \%$ C.L. exclusion limits for LHC at $14 \mathrm{TeV}$ with $\mathcal{L}=300 \mathrm{fb}^{-1}$ (dashed-black) and $\mathcal{L}=3000 \mathrm{fb}^{-1}$ (HL-LHC, dotted-black) assuming the measured Higgs signal strengths being $\mu_{i}=1$ and using the projected CMS sensitivities from section 3.1.

We now analyze the constraints from EWPO, performing a fit to the oblique parameters $S, T$ under the assumption $U=0$ using the best-fit values and standard deviations from the global analysis of the GFitter Group [72] $\left(m_{t}=173 \mathrm{GeV}\right.$ and $\left.m_{h}=126 \mathrm{GeV}\right)$, as shown in (3.17). The $2 \mathrm{HDM}$ contributions to $\Delta S$ and $\Delta T$ are given by [118]

$$
\begin{aligned}
\Delta S= & \frac{g^{2} s_{W}^{2}}{96 \pi^{2} \alpha_{\mathrm{EM}}}\left[\left(1-2 s_{W}^{2}\right)^{2} G_{H^{ \pm}, H^{ \pm}, Z}+G_{A^{0}, H^{0}, Z}+\log \left(m_{A^{0}}^{2} m_{H^{0}}^{2} / m_{H^{ \pm}}^{4}\right)\right. \\
& \left.+c_{\beta-\alpha}^{2}\left(G_{A^{0}, h, Z}-G_{A^{0}, H^{0}, Z}+\hat{G}_{H^{0}, Z}-\hat{G}_{h, Z}\right)\right]
\end{aligned}
$$


Type I

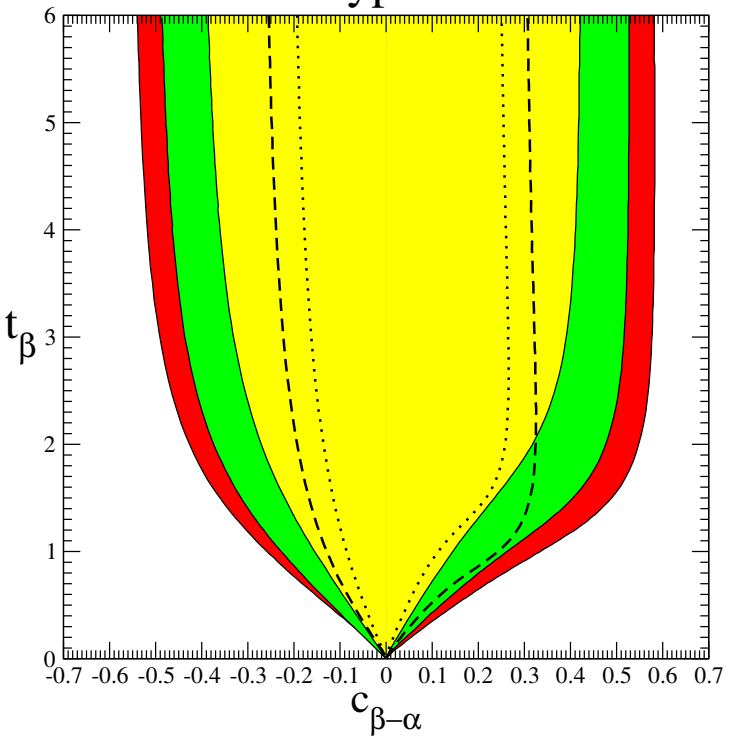

Lepton Specific

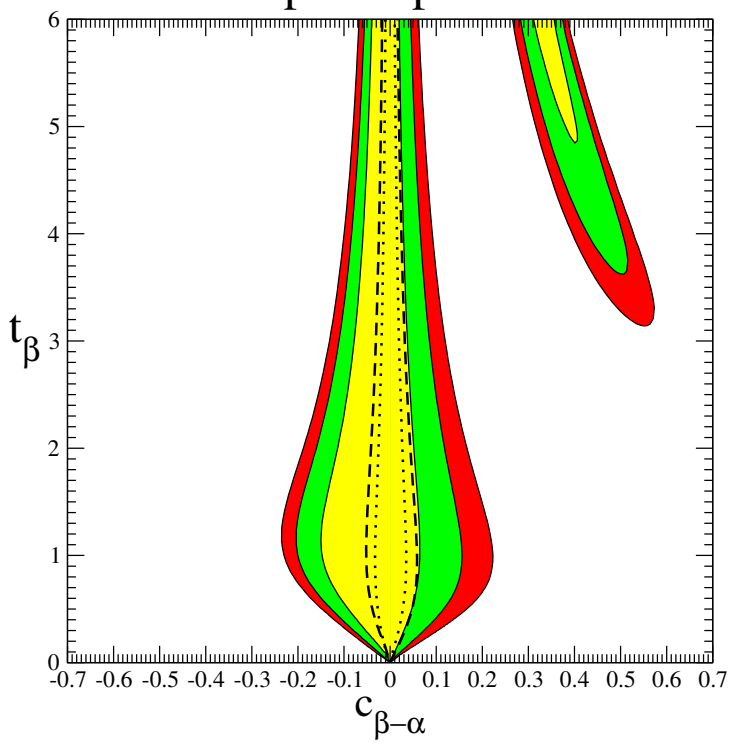

Type II

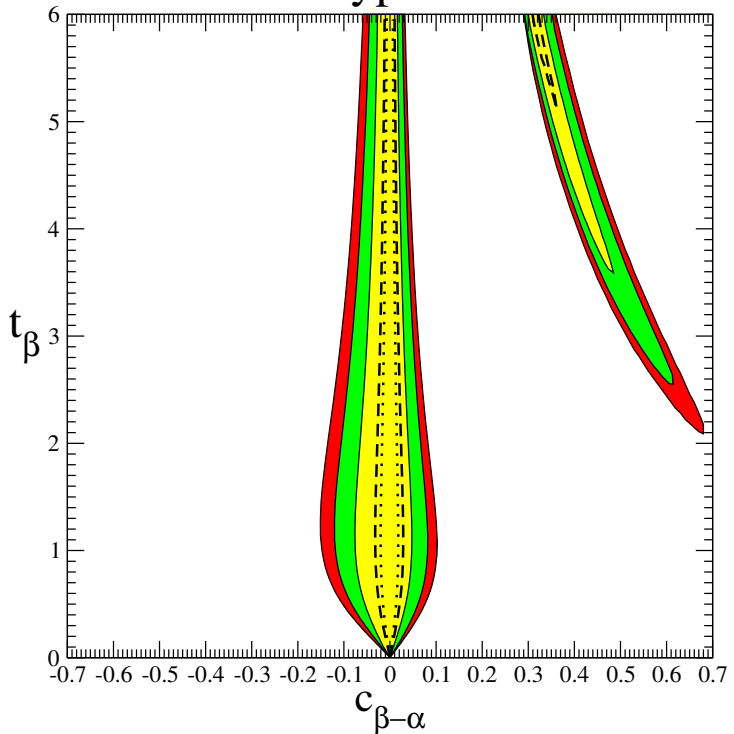

Flipped

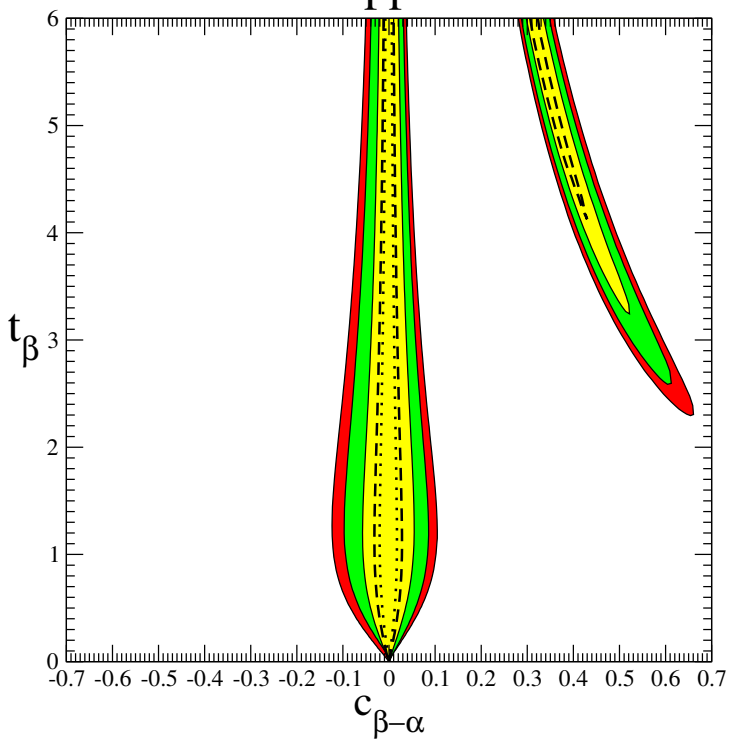

Figure 5. $68 \%$ C.L. (yellow), $95 \%$ C.L. (green) and $99 \%$ C.L. (red) allowed regions in the ( $\cos (\beta-$ $\alpha), \tan \beta$ ) plane from up-to-date measurements of Higgs boson couplings (see text for details), shown respectively for 2HDM of Type I (Upper-Left), Type II (Upper-Right), Lepton-Specific (Lower-Left) and Flipped (Lower-Right). In each case, the projected 95\% C.L. exclusion limit for LHC at $14 \mathrm{TeV}$ with $\mathcal{L}=300 \mathrm{fb}^{-1}$ (dashed-black) and $\mathcal{L}=3000 \mathrm{fb}^{-1}$ (dotted-black), assuming the measured Higgs signal strengths being $\mu_{i}=1$, is also shown. 

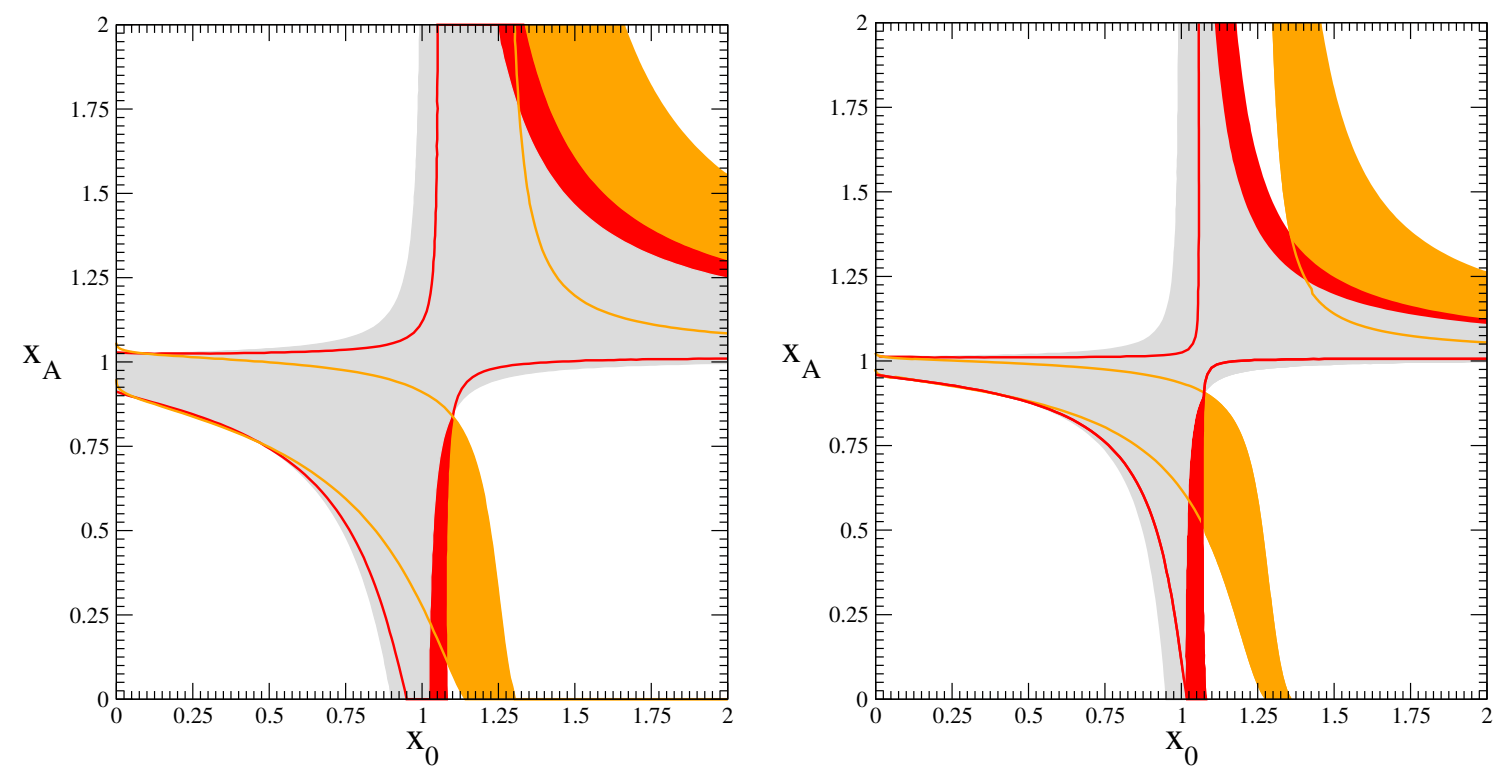

Figure 6. $95 \%$ C.L. allowed region from the $2 \mathrm{HDM}$ fit to $S, T$ oblique parameters (3.53) in the plane $\left(x_{0}, x_{A}\right)$ for $m_{H^{ \pm}}=400 \mathrm{GeV}$ (Left) and $m_{H^{ \pm}}=600 \mathrm{GeV}$ (Right). The allowed regions correspond respectively to $c_{\beta-\alpha}=0$ (grey, light), $c_{\beta-\alpha}=0.2$ (red, dark) and $c_{\beta-\alpha}=0.4$ (orange, medium).

$$
\begin{aligned}
\Delta T= & \frac{F_{H^{ \pm}, A^{0}}+F_{H^{ \pm}, H^{0}}-F_{A^{0}, H^{0}}}{16 \pi^{2} v^{2} \alpha_{\mathrm{EM}}}+c_{\beta-\alpha}^{2} \frac{F_{H^{ \pm}, h}-F_{H^{ \pm}, H^{0}}+F_{A^{0}, H^{0}}-F_{A^{0}, h}}{16 \pi^{2} v^{2} \alpha_{\mathrm{EM}}} \\
& +c_{\beta-\alpha}^{2} \frac{3\left(F_{H^{0}, Z}-F_{H^{0}, W}-F_{h, Z}+F_{h, W}\right)}{16 \pi^{2} v^{2} \alpha_{\mathrm{EM}}}
\end{aligned}
$$

with $G_{A, B, C}, \hat{G}_{A, B}$ and $F_{A, B}$ given in appendix A.2. We note that both $\Delta S$ and $\Delta T$ are independent of $t_{\beta}$. We define

$$
x_{0} \equiv \frac{m_{H^{0}}^{2}}{m_{H^{ \pm}}^{2}}, x_{A} \equiv \frac{m_{A^{0}}^{2}}{m_{H^{ \pm}}^{2}},
$$

and construct a $\Delta \chi_{E W}^{2}$ function as

$$
\Delta \chi_{E W}^{2}\left(m_{H^{ \pm}}^{2}, x_{0}, x_{A}, c_{\beta-\alpha}\right)=\sum_{i, j}\left(\Delta \mathcal{O}_{i}-\Delta \mathcal{O}_{i}^{0}\right)\left(\sigma^{2}\right)_{i j}^{-1}\left(\Delta \mathcal{O}_{j}-\Delta \mathcal{O}_{j}^{0}\right)
$$

with $\Delta \mathcal{O}_{i}^{0}$ and $\left(\sigma^{2}\right)_{i j}$ defined after (3.19). The results of the fit are shown in figure 6 for various values of $c_{\beta-\alpha}$ and $m_{H^{ \pm}}=400,600 \mathrm{GeV}$.

The calculation of the oblique $S$ and $T$ parameters in the 2HDM matches with the $D=6$ effective operators present in the EFT. Considering first the alignment limit and expanding (3.50) and (3.51) around $x_{0}, x_{A} \sim 1$ (as indicated by the present 95\% C.L. experimental limits), $\Delta S$ and $\Delta T$ in the $2 \mathrm{HDM}$ read

$$
\begin{aligned}
\Delta S & =\frac{g^{2} s_{W}^{2}}{96 \pi^{2} \alpha_{\mathrm{EM}}} \log \left(x_{0} x_{A}\right) \simeq-\frac{g^{2} s_{W}^{2}\left(1-x_{A}+1-x_{0}\right)}{96 \pi^{2} \alpha_{\mathrm{EM}}} \\
\Delta T & =\frac{m_{H^{ \pm}}^{2}\left(1-x_{A}\right)\left(1-x_{0}\right)}{48 \pi^{2} v^{2} \alpha_{\mathrm{EM}}}
\end{aligned}
$$


where we note that the functions $G_{A, B, C}$ scale as $\left(1-x_{A, 0}\right)^{2}$ and can be neglected with respect to the $\log \left(x_{0} / x_{A}\right)$ term in $\Delta S$. By means of (A.14) and (A.15) in appendix A.1, the above relations can be rewritten as

$$
\begin{aligned}
\frac{\alpha_{E M}}{4 s_{W}^{2}} \Delta S & =\frac{m_{W}^{2}}{96 \pi^{2}} \frac{\tilde{\lambda}_{4}}{m_{H^{ \pm}}^{2}} \\
\alpha_{\mathrm{EM}} \Delta T & =\frac{v^{2}}{192 \pi^{2}} \frac{\tilde{\lambda}_{4}^{2}-\tilde{\lambda}_{5}^{2}}{m_{H^{ \pm}}^{2}}
\end{aligned}
$$

For $m_{H^{ \pm}}^{2} \gg v^{2}$, and upon the replacement $m_{H^{ \pm}}^{2} \rightarrow \tilde{\mu}_{2}^{2}$, these match the EFT results (3.28)

$$
\begin{aligned}
\bar{c}_{W}+\bar{c}_{B} & =\frac{m_{W}^{2}}{96 \pi^{2}} \frac{\tilde{\lambda}_{4}}{\tilde{\mu}_{2}^{2}}=\frac{\alpha_{E M}}{4 s_{W}^{2}} \Delta S=\epsilon_{3} \\
\bar{c}_{T} & =\frac{v^{2}}{192 \pi^{2}} \frac{\tilde{\lambda}_{4}^{2}-\tilde{\lambda}_{5}^{2}}{\tilde{\mu}_{2}^{2}}=\alpha_{\mathrm{EM}} \Delta T=\epsilon_{1}
\end{aligned}
$$

where we have also translated into the Altarelli-Barbieri parametrization [119-121] for reference. The bounds on these effective operators are [72]

$$
\bar{c}_{T}\left(m_{Z}\right) \in[-1.5,2.2] \times 10^{-3} \quad \text { and } \quad\left(\bar{c}_{W}\left(m_{Z}\right)+\bar{c}_{B}\left(m_{Z}\right)\right) \in[-1.4,1.9] \times 10^{-3} .
$$

Away from alignment, $\Delta S$ and $\Delta T$ receive further contributions $\propto c_{\beta-\alpha}^{2}$. In an EFT language, these are captured via $R G$ evolution: various operators from (3.40) may mix with $\mathcal{O}_{W}, \mathcal{O}_{B}$ and $\mathcal{O}_{T}$ via $\mathrm{RG}$ running, as is the case of e.g. $\mathcal{O}_{H}$ [28], whose mixing at leading order would yield

$$
\begin{aligned}
\bar{c}_{T}\left(m_{Z}\right) & \simeq \bar{c}_{T}\left(\tilde{\mu}_{2}\right)-\frac{3 g^{\prime 2}}{32 \pi^{2}} \bar{c}_{H}\left(\tilde{\mu}_{2}\right) \log \left(\frac{\tilde{\mu}_{2}}{m_{Z}}\right) \\
\bar{c}_{W}\left(m_{Z}\right)+\bar{c}_{B}\left(m_{Z}\right) & \simeq c_{W}\left(\tilde{\mu}_{2}\right)+\bar{c}_{B}\left(\tilde{\mu}_{2}\right)+\frac{1}{24 \pi^{2}} \bar{c}_{H}\left(\tilde{\mu}_{2}\right) \log \left(\frac{\tilde{\mu}_{2}}{m_{Z}}\right) .
\end{aligned}
$$

However, for the $2 \mathrm{HDM} \bar{c}_{H}\left(\tilde{\mu}_{2}\right)=0$ at tree-level, and thus the Wilson coefficient responsible for the contribution to $\Delta S$ and $\Delta T$ dependant on $c_{\beta-\alpha}^{2}$ is $\bar{c}_{6 h}[27]$.

\subsubsection{Alignment limit: complementarity of EWPTs and LHC limits}

In the previous section we discussed how LEP electroweak constraints translate into the full theory (3.28), and in turn in the effective theory (3.56) which affected the operators $\bar{c}_{T}$ and a combination $\bar{c}_{W}+\bar{c}_{B}$. It is time to move onto the constraints from LHC, the most stringent ones on operators affecting the decay of the Higgs to photons $\left(\bar{c}_{\gamma}\right)$ and production through gluon fusion $\left(\bar{c}_{g}\right)$. Other operators are better determined by looking at other production and decay channels. In particular, three combinations [19]

$$
\bar{c}_{W}-\bar{c}_{B}, c_{H W} \text { and } \bar{c}_{H B},
$$

are constrained with the help of Higgs production in association with a vector boson, and with diboson data [21]. In the $2 \mathrm{HDM}$, the global fit is more constraining than a fit for a 


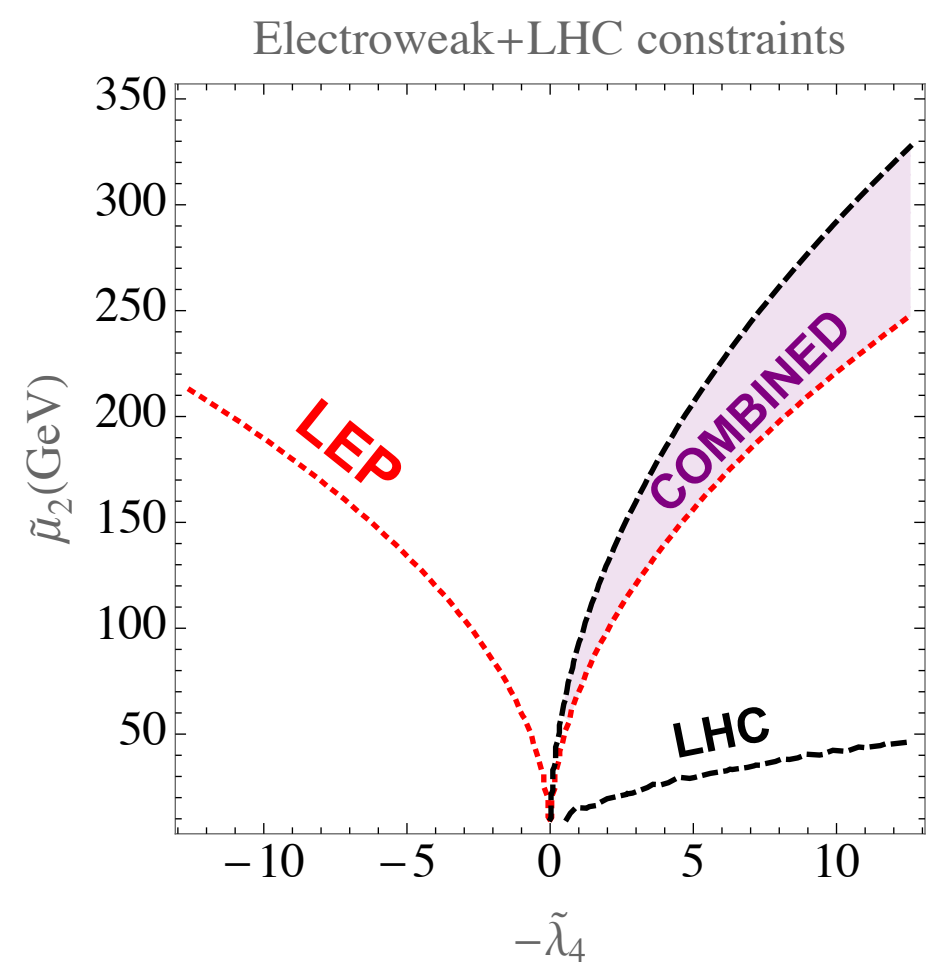

Figure 7. $95 \%$ C.L. limits on the $2 \mathrm{HDM}$ parameters $\tilde{\lambda}_{4}$ and $\tilde{\mu}_{2}$. The red-dotted lines enclose the region allowed by constraints on the $S$ parameter, with the black-dashed lines the corresponding LHC limits from [21]. The purple-solid region corresponds to the combined limits.

general EFT at dimension-six level. In particular, by varying all the free parameters in the 2HDM simultaneously in a fit to Higgs plus diboson data, one obtains the following $95 \%$ C.L. regions $[21]$

$$
\begin{aligned}
\bar{c}_{H W} & \in(0.0004,0.02) \\
\bar{c}_{g} & \in-(0.00004,0.000003) \\
\bar{c}_{\gamma} & \in(-0.0006,0.00003)
\end{aligned}
$$

Whereas the constraint on the $T$ parameter favours a $\tilde{\lambda}_{5}$ close to $\tilde{\lambda}_{4}$, the constraint on $\bar{c}_{\gamma}$ from the LHC restricts the values of $\tilde{\lambda}_{3}$. The most interesting case is when we compare constraints from LEP and LHC which set limits on the coupling $\lambda_{4}$ and the scale of new physics. Indeed, one can see this interplay between the two sources of data in figure 7 , where we present results in the $\tilde{\lambda}_{4}$ and $\tilde{\mu}_{2}$ plane. The red-dotted lines enclose the region allowed by constraints on the $S$ parameter, and the black-dashed lines are the corresponding LHC limits from [21]. The purple-solid region corresponds to the combined limits and it is quite constraining as the two regions have little overlap. Note that the preferred region at $95 \%$ C.L. is consistent with the presence of light particles. This is due to the slightly non-zero positive value of $\bar{c}_{H W}$ in the global LHC fit and the tension with LEP limits, which favour negative values of $\bar{c}_{H W}$. This hint for new physics is gone at the level of $3 \sigma$. 
Finally, we comment on the 2HDM and the impact on the EW phase transition. As recently shown in [111], a first order EW phase transition strongly favours a large $m_{A^{0}}-m_{H^{0}}$ splitting, which via (A.17) implies $\tilde{\lambda}_{5}<0$ and sizable. Since this implies that it is not possible to simultaneously have vanishing $\Delta S$ and $\Delta T$ unless $\tilde{\mu}_{2} \rightarrow \infty$, it would be possible to observe a deviation from the $\mathrm{SM}$ in $\Delta S$ or $\Delta T$ as the experimental precision increases (see figure 8).

\subsection{A dilaton/radion scenario}

So far we have discussed scenarios where the operators involving the Higgs and massive vector bosons, such as $\bar{c}_{W}$, are generated at loop level. In this section we present a case where they appear at tree-level, through the exchange of a dilaton or a radion scalar particle. We consider two equivalent scenarios:

- The extra-dimensional radion: a radion $r$ is the excitation of the graviton perturbation in extra-dimensions along the direction of the extra-dimension. The interactions of the radion with SM particles are then obtained by expanding the metric at linear order in $r$. We will consider conformal metrics of the form

$$
d s^{2}=w(z)^{2}\left(\eta_{\mu \nu} d x^{\mu} d x^{\nu}-d z^{2}\right)
$$

where $w(z)$ is the warp factor, which depends on the extra-dimension $z$. Minkowski space corresponds to $w=1$ and in Anti-deSitter metrics $w=1 /(k z)$, with $k$ the curvature in the extra-dimension. The extra-dimension is compactified, with $z$ stretching between two points which we will call the UV and IR branes, i.e. $z \in\left[z_{U V}, z_{I R}\right]$. The interaction then reads

$$
\mathcal{S}=\int d^{d} x \sqrt{-g} \mathcal{L} \supset \int d^{d} x \sqrt{-g} w^{2}(z) 2 r T_{\mu}^{\mu}
$$

where $T_{\mu}^{\mu}$ is the trace of the stress-energy tensor. The dimensional reduction of the interaction in (3.63) to a four-dimensional (4D) interaction depends on the localization of the radion and the SM particles, and generally speaking it would look as

$$
\mathcal{L}_{4 D}=\frac{c_{i}}{\sqrt{6} \Lambda} r T^{i}
$$

where now $r$ and $T^{i}=\operatorname{Tr}\left(T_{\mu \nu}^{i}\right)$ are the dimensionally-reduced radion and trace of the stress-energy tensor of species $i$. $\Lambda$ corresponds to the compactification scale, and the coefficients $c_{i}$ account for the overlap of different bulk species $i$ with the radion.

In warped extra-dimensions, the radion is a field localized near the IR brane at $z_{I R}$. Therefore, for fields localized near the IR brane, $c_{i} \simeq \mathcal{O}(1)$, for fields delocalized in the bulk (with a flat profile) one finds $c_{i} \simeq \frac{1}{\int_{I_{I R}}^{z} w(z) d z}$ and for fields on the UV brane, $c_{i}\left(\frac{z_{U V}}{z_{I R}}\right)^{a}$ with $a$ a positive number [122-124]. As an example, in AntideSitter (AdS) models, the suppression for bulk fields is $\int w(z) d z=\log \frac{z_{U V}}{z_{I R}}$, which in Randall-Sundrum translates into $\log \left(\mathrm{M}_{\mathrm{P}} / \mathrm{TeV}\right) \simeq \mathcal{O}(30)$. 
Although massless gauge fields do not contribute to the trace of the stress-energy tensor at tree-level, loop-level couplings are generated by the trace anomaly

$$
T_{\mu, \text { anom }}^{\mu}=-\sum_{i} \frac{b_{i} \alpha_{i}}{8 \pi} F_{\mu \nu}^{i} F^{i \mu \nu}
$$

where $i$ runs over the gauge groups of the $\mathrm{SM}, \mathrm{SU}(3)_{c} \times \mathrm{SU}(2)_{L} \times \mathrm{U}(1)_{Y}, \alpha_{i}=g_{i}^{2} / 4 \pi$ and $g_{i}$ are the respective coupling constants. The $b_{i}$ are the $\beta$ function coefficients leading to anomalous scale-invariance violations (dilaton) or, equivalently, contributions from fields localized near the IR brane or bulk running (radion) [125-127]. The values of $b_{i}$ depend on the degree of compositeness of fermions in the SM and possible new CFT contributions. For simplicity we neglect these and use the pure CFT value, $b_{i}^{C F T}=8 \pi^{2} /\left(g_{i}^{2} \log \left(z_{U V} / z_{I R}\right)\right)$. In this limit the coefficient of the anomaly is then independent of the value of the gauge coupling

$$
T_{\mu, \text { anom }}^{\mu}=-\sum_{i} \frac{\log \left(z_{I R} / z_{U V}\right)}{4} F_{\mu \nu}^{i} F^{i \mu \nu}
$$

- The dilaton of spontaneous breaking of scale invariance: assume the existence of a new sector whose couplings are scale invariant, but this scale invariance is spontaneously broken by some dynamics. For example, in QCD scale invariance is spontaneously broken by confinement dynamics, leading to quark condensates. In our example, we are considering a new strongly coupled sector, possibly with similar dynamics as QCD. The Goldstone boson of this spontaneous breaking is a dilaton. Let us denote the dilaton by $r$ as in the radion case. Non-derivative couplings of the dilaton to SM fields are proportional to the explicit breaking of conformal invariance, namely they are of the form

$$
\frac{r}{f} \partial_{\mu} J^{\mu}
$$

with $f$ the scale of the spontaneous breaking of dilatation symmetry and $J^{\mu}$ the global conserved current for this symmetry, $J^{\mu}=T^{\mu \nu} x_{\nu}$. Therefore the coupling of the dilaton to the SM fields take the same form as those of the radion (3.64) [128]. We stress that the quadratic couplings to SM particles do not have the same form for the radion and dilaton [128], but this difference will not affect the matching of dimension-six coefficients.

The radion/dilaton mass is a model-dependent parameter, related to the mechanism of stabilization of the extra-dimension or the explicit breaking of dilatation symmetry. In absence of stabilization the radion is massless but one could stabilize this dilaton/radion in several ways, and no definite prediction of the mass can be drawn unless we specify the mechanism. For example, in the Goldberger-Wise mechanism [129] in warped extradimensions the radion mass is a function of the vev and mass of the stabilizing field [130] and could be very light as well as around the scale of compactification (or spontaneous breaking) $f$. 
We proceed to integrate out the dilaton assuming its mass $m_{r}$ is larger than the scales we are probing with colliders. The effective Lagrangian has the form

$$
\mathcal{L}_{\text {eff }}=-\frac{1}{f^{2}} \frac{1}{m_{r}^{2}} T^{2}
$$

where $T$ is the trace of the stress-energy tensor. For the Higgs and gauge bosons,

$$
T \subset-2\left|D_{\mu} \Phi\right|^{2}+4 V\left(\Phi^{\dagger} \Phi\right)-\frac{b_{i} \alpha_{i}}{8 \pi} F_{\mu \nu}^{i} F^{i \mu \nu}
$$

where $V=-m_{h}^{2}|\Phi|^{2}+\lambda|\Phi|^{4}$. One can then extract the Wilson coefficients of the effective operators

$$
\begin{aligned}
\bar{c}_{H} & =-\bar{c}_{6}=8 \frac{m_{h}^{2} v^{2}}{f^{2} m_{r}^{2}} \\
\bar{c}_{H W} & =-\bar{c}_{W}=-\frac{b_{2} \alpha_{2}}{4} \frac{m_{h}^{2} v^{2}}{f^{2} m_{r}^{2}} \\
\bar{c}_{H B} & =-\bar{c}_{B}=-\frac{b_{1} \alpha_{1}}{4} \frac{m_{h}^{2} v^{2}}{f^{2} m_{r}^{2}} \\
\bar{c}_{\gamma} & =-\frac{b_{1} \alpha_{1}}{4 \pi} \frac{g^{2} m_{h}^{2} v^{2}}{g^{\prime 2} f^{2} m_{r}^{2}} \quad \bar{c}_{g}=-\frac{b_{3} \alpha_{3}}{4 \pi} \frac{g^{2} m_{h}^{2} v^{2}}{g_{s}^{2} f^{2} m_{r}^{2}}
\end{aligned}
$$

We stress again that for a pure CFT value $b_{i}^{C F T}, b_{1} \alpha_{1}=b_{2} \alpha_{2}=b_{3} \alpha_{3}$. We could also consider a more general situation, where the coupling of the dilaton/radion is not universal. This amounts to setting different values of the coefficients $c_{i}$ in (3.64) and would lead to a prefactor in the coefficients of the effective operators (3.70), dependent of the degree of overlap of the wavefunctions in the bulk (radion) or participation on the composite dynamics of the species (dilaton).

While the contributions to $\Delta T$ vanish in this case at tree-level [131, 132], they will be generated both at 1-loop (this is also the case for $\Delta S$ ) and through operator mixing due to RG running. The 1-loop contribution to $\Delta T$ and $\Delta S$ is given by [131]

$$
\bar{c}_{T}=\frac{g^{2} s_{W}^{2}}{64 \pi^{2} c_{W}^{2}} \frac{v^{2}}{f^{2}} \log \left(\frac{f}{m_{r}}\right) \quad, \quad \bar{c}_{W}+\bar{c}_{B}=-\frac{g^{2} v^{2}}{(24 \pi)^{2} f^{2}} \log \left(\frac{f}{m_{r}}\right)
$$

The corresponding contributions to $\bar{c}_{T}\left(m_{Z}\right)$ and $\bar{c}_{W}\left(m_{Z}\right)+\bar{c}_{B}\left(m_{Z}\right)$ from the RG running of $\bar{c}_{H}$ are given by (3.58)-(3.59) with the substitution $\tilde{\mu}_{2} \rightarrow m_{r}$ and using $\bar{c}_{H}\left(m_{r}\right)$ from (3.70). We note that the RG running gives a contribution to $\Delta T$ and $\Delta S$ which despite begin suppressed by $m_{h}^{2} / m_{r}^{2}$ w.r.t. the 1-loop contribution (3.71), may become dominant due to a much smaller numerical suppression. The fact that the two contributions have opposite signs leads to a partial cancellation effect. In figure 8 we show the results on the $\Delta S-\Delta T$ plane, and compare the correlation in the oblique parameters with that appearing in the ones encountered respectively in the Higgs portal and 2HDM scenarios from section 3.1 and 3.2 . 

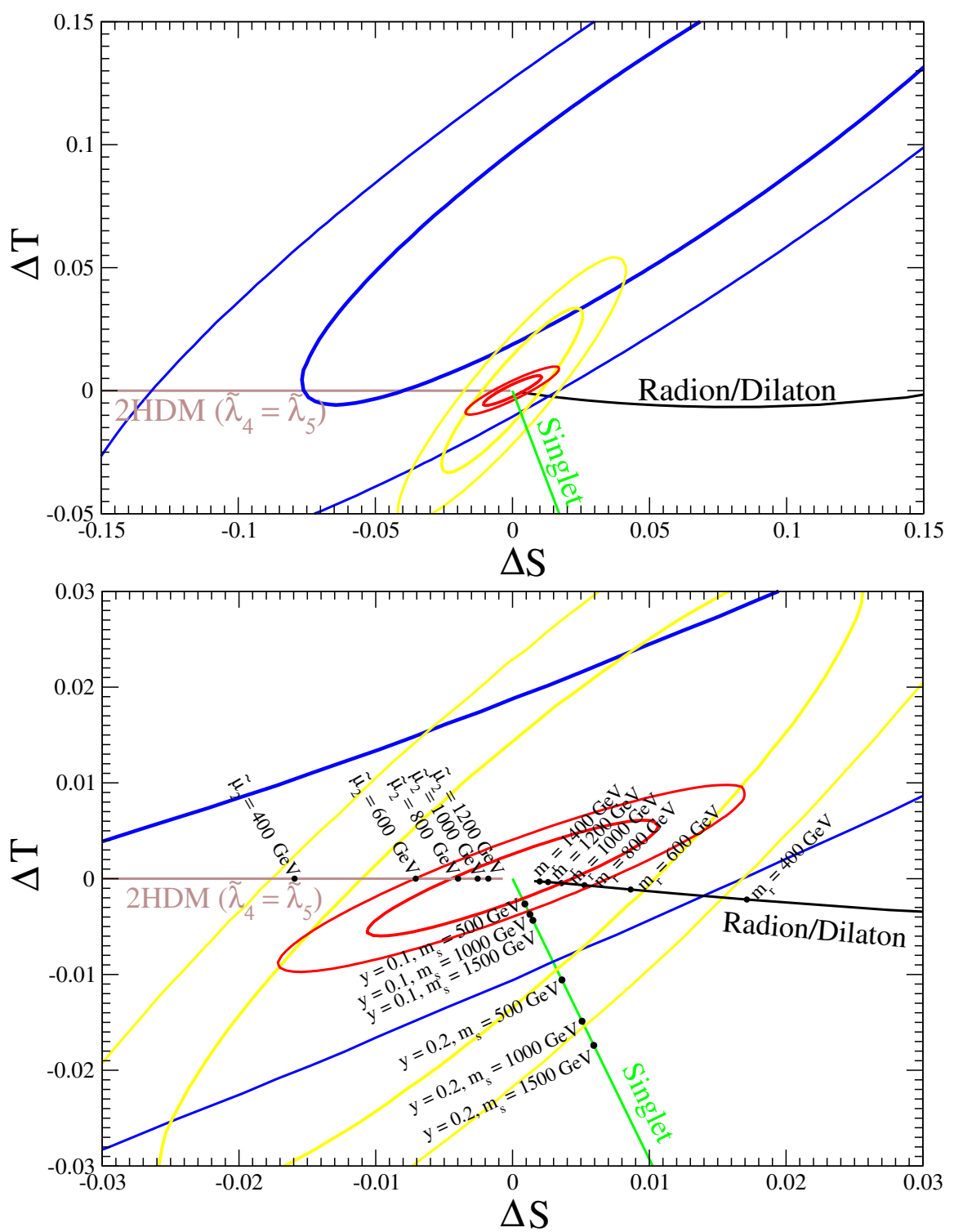

Figure 8. $\Delta S-\Delta T$ constraints on the radion/dilaton (black), singlet-Higgs portal (green) and $2 \mathrm{HDM}$ w. custodial symmetry (brown) scenarios. The thick (thin) ellipses correspond to the $68 \%$ C.L. (95\% C.L.) allowed region for LEP (blue) and the projected ILC Giga-Z (yellow) and TLEP TeraZ (red), see section 3.1 for more details. The zoom on the percent region (Bottom) includes points for the Higgs portal with various values of mixing parameter $y$ and mass $m_{s}$, for the radion/dilaton with $f=2 \mathrm{TeV}$ and various values of the radion mass $m_{r}$, and for the $2 \mathrm{HDM}$ with $\tilde{\lambda}_{4}=\tilde{\lambda}_{5}=-\pi$ and several values of $\tilde{\mu}_{2}$. 


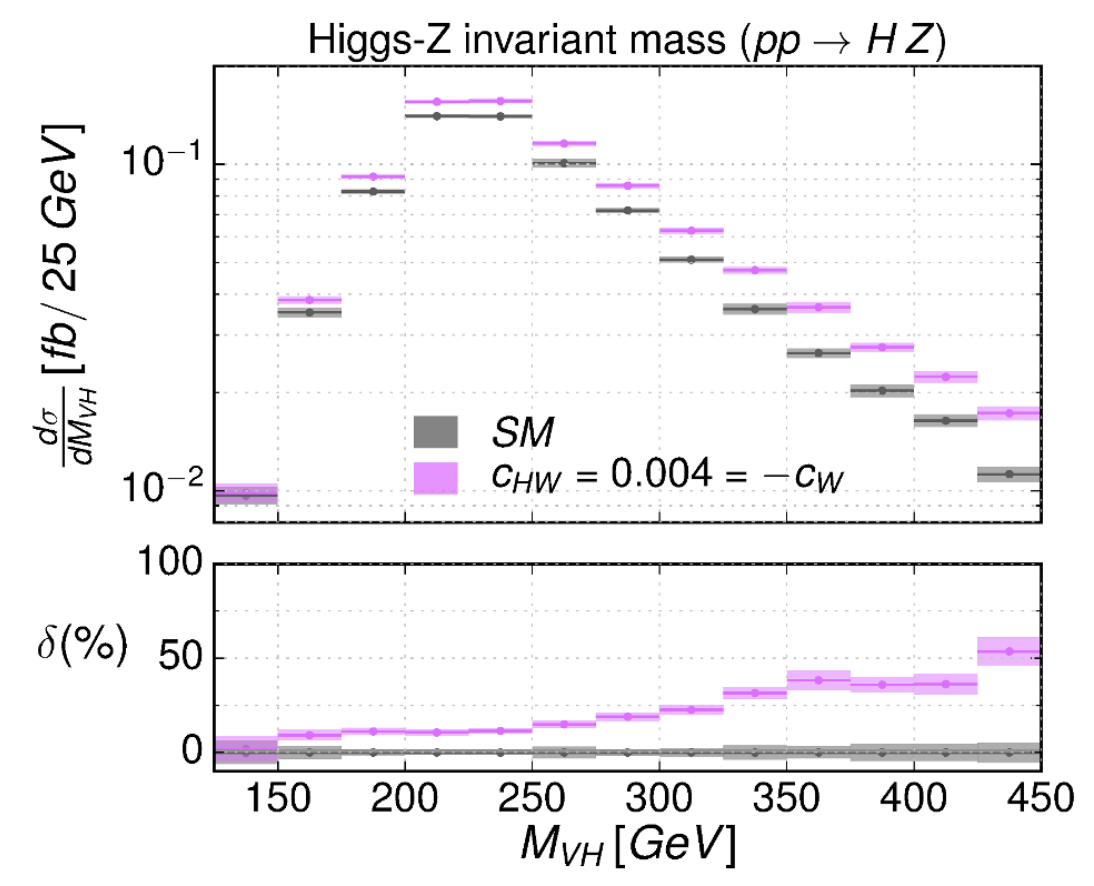

Figure 9. Higgs produced in association with a vector boson: invariant mass distribution. Grey and pink distributions correspond to SM and EFT with $\bar{c}_{H W}=-\bar{c}_{W}=0.004$. The width correspond to varying the factorization scale $\mu=m_{h}+m_{Z}$ in a range of $2 \mu$ and $\mu / 2$. The value of $\bar{c}_{W}$ is consistent with Run1 data and will be explored in the Run2 LHC. The value $\delta$ is the deviation respect to the SM central value.

\section{The validity of the EFT: from operators to total rates and distributions}

The EFT approach, where higher-dimensional operators are written as a way to encode effects of New Physics, is a good approximation at low energies and should not be used in an arbitrary range of energies. Indeed, as these operators are suppressed by some New Physics scale, parton level cross-sections would diverge with the parton energy.

This growth with energy is a signature that the EFT approach breaks down when one is able to probe the dynamics of the heavy particles one integrates out. In hadron colliders, this corresponds to the moment when the partonic energy $\sqrt{\hat{s}}$ is comparable with the masses in the loop. Specifically, for a tree-level exchange as in figure 1,

$$
\sqrt{\hat{s}} \simeq M
$$

The same argument applies to the dilaton exchange, and to fermion couplings in 2HDM as in figure 4. On the other hand, in loop-induced processes as in figure 10 the validity of the EFT extends to the threshold to produce a pair of new particles, namely

$$
\sqrt{\hat{s}} \simeq 2 M \text {. }
$$

How this energy is related to the strength of the dimension-six deviation from the SM depends on the model. Let us focus on operators of the type $\bar{c}_{W}$ and $\bar{c}_{H W}$ which 
contain derivatives on the Higgs field. We have found the matching with the 2HDM and radion-exchange is roughly speaking

$$
\frac{\bar{c}}{m_{W}^{2}} \sim \frac{\lambda}{192 \pi^{2}} \frac{1}{M^{2}}(2 H D M) \text {, and } \frac{m_{h}^{2}}{\Lambda^{2}} \frac{1}{M^{2}} \text { (radion/dilaton), }
$$

where $M$ plays the role of the mass of the extra Higgses and dilaton, $\lambda$ denotes a combination of quartic couplings in the Higgs potential, and $\Lambda$ an effective scale suppression for the dimension-five interaction between the dilaton and the SM particles, $\Lambda>M$.

At energies around the mass threshold, the EFT description should be substituted by the full UV theory including the resonance dynamics. The question we would like to ask is what is the deviation of the EFT predictions from the full UV model, which depends on the process and distribution one is looking at. A full comparison of the UV theory and the EFT results is beyond the scope of this paper, but in this section we would like to discuss the qualitative features of this deviation.

To do so, we present an example of a specially useful distribution [133, 134] to probe New Physics, namely the invariant mass of the Higgs and vector boson system in Higgs associated production, $M_{V H}$, as it corresponds to the parton energy, $\hat{s}=M_{V H}^{2}$. In figure 9 we show how the invariant mass distribution changes when dimension-six operators are included. Grey and pink distributions correspond to SM and EFT with $\bar{c}_{H W}=-\bar{c}_{W}=$ 0.004. The width correspond to varying the factorization scale $\mu=m_{h}+m_{Z}$ in a range of $2 \mu$ and $\mu / 2$. The value of $\bar{c}_{W}$ is consistent with Run1 data and will be explored in the Run2 LHC. The value $\delta$ is the deviation respect to the SM central value.

With the rough estimate given in eq. (4.2), one can see that for a $2 \mathrm{HDM}$, with $\lambda=$ $3 \times 4 \pi$ the breakdown occurs at around $M_{V H} \simeq 360 \mathrm{GeV}$. For the dilaton, in the case of $\Lambda=500 \mathrm{GeV}$, the breakdown occurs at about $320 \mathrm{GeV}$. These numbers highlight the importance to relate EFT distributions with specific UV models.

The distributions are the result of a simulation including PDF effects (NN23L01) and parton-showering and hadronization performed using an implementation from MCFM [135139] into POWHEG [140] at NLO in QCD. We have checked that these results are consistent with a parallel implementation in MG5 MC@NLO [141] using the model implementation in [142] into NLO QCD, based on Feynrules [143] and the UFO [144] format. Note that the EFT distribution does not grow with $\sqrt{\hat{s}}$, but falls down due to the PDF effects.

Besides this distribution, one can find others sensitive to New Physics effects, e.g. $m_{j j}$ and $\Delta y_{j j}$ in Vector Boson Fusion [145] (see also [146]), or $\Delta R_{h h}$ and $p_{T}^{h h}$ in di-Higgs production [147].

\section{Discussion and summary}

During Run2 of the LHC, interpretation of data using the Effective Field Theory (EFT) approach will become a standard way to communicate results to theorists, as the translation to UV models is more direct than, for example, the use of form factors.

The approach based on anomalous couplings and the one considered here are related. New Physics affects the behaviour of SM particles, inducing anomalous couplings, for example involving the Higgs and electroweak bosons. In the context of heavy New Physics, the 
anomalous couplings can be translated into combinations of higher-dimensional operators as shown in tables 5 and 6 .

The use of the EFT approach, however, is limited to heavy new physics, whereas current LHC limits strive to reach the $\mathrm{TeV}$ region, leading to a delicate balance between using as much data as possible and the model-independent EFT approach. The question of what LHC data is suitable to constrain EFTs depends both on the UV completion and the type of signal one is looking at. Benchmark models allow to address these issues, and also to draw correlations with direct searches and other non-LHC sources of indirect constraints such as flavour physics and LEP. In this paper we have taken a first step towards this program by studying the matching between UV completions encompassing extensions of the Higgs sector and the EFT.

This matching allowed us to study the suitability of LHC data to constrain EFTs. For example, one can consider UV completions with large couplings where the validity of the EFT is improved, such as the $2 \mathrm{HDM}$ in section 3.2 with large quartic couplings. But even for weakly-coupled UV completions, the use of the EFT may be justified as long as the LHC data one uses is restricted to small values of $\sqrt{\hat{s}}$, below the masses of new particles, as discussed in section 4 .

We have shown the matching of the UV theory with the low-energy coefficients in detail. This matching is straightforward in the case of the tree-level dilaton exchange (section 3.3) or mixing with a singlet (section 3.1). For loop-induced dimension-six operators, however, the interplay of with higher order terms (dimension-eight operators) needs to be handled with care as shown in section 3.2.2.

Another advantage of the comparison with UV completions is to address correlations among the EFT coefficients which are present in models and reduce the number of free parameters in a global analysis. For example, in our benchmarks specific relations among operators can be traced back to the (limited) Lorentz structures one can build up from scalar fields, see discussion around (3.28).

Note that we have not discussed the very interesting possibility of CP-violating effects from, for example, complex parameters in the 2HDM. This deserves further study, as their kinematic distributions and dependence with energy have to be simulated as close as possible to the actual cuts applied by the LHC collaborations.

Note added: as this work was being submitted for publication, we became aware of [151], which also discusses UV completions of Higgs Effective Field Theory via extended Higgs sectors finding results similar to ours. We stress that both works are complementary: we focus on the connection between the EFT and UV models as a way to assess the range of validity of the EFT, whereas [151] discusses the connection between the EFT and UV models in the context of a systematic framework for the obtention of the EFT Wilson coefficients from an arbitrary UV theory.

\section{Acknowledgments}

We would like to thank Ken Mimasu for help on the QCD NLO simulation. V.S and J.M.N. acknowledge support from the Science and Technology Facilities Council (STFC) 
through the grants ST/J000477/1 and ST/L000504/1. The work of M.G. is partially supported by the STFC consolidated grant ST/L000431/1. J.M.N. is supported by the People Programme (Marie curie Actions) of the European Union Seventh Framework Programme (FP7/2007-2013) under REA grant agreement PIEF-GA-2013-625809.

\section{A 2HDM results and conventions}

\section{A.1 Scalar potential and mass spectrum}

Let us recall the scalar potential for a $2 \mathrm{HDM}$ with a softly-broken $\mathbb{Z}_{2}$-symmetry in the CPconserving case, given by (3.25). After EW symmetry breaking, the scalar mass eigenstates can be written in terms of the original fields in (3.26) as

$$
\begin{aligned}
G^{ \pm} & =\cos \beta \varphi_{1}^{ \pm}+\sin \beta \varphi_{2}^{ \pm} & H^{ \pm} & =-\sin \beta \varphi_{1}^{ \pm}+\cos \beta \varphi_{2}^{ \pm} \\
G^{0} & =\cos \beta \eta_{1}+\sin \beta \eta_{2} & A^{0} & =-\sin \beta \eta_{1}+\cos \beta \eta_{2} \\
h & =-\sin \alpha h_{1}+\cos \alpha h_{2} & H^{0} & =-\cos \alpha h_{1}-\sin \alpha h_{2}
\end{aligned}
$$

with $H^{ \pm}, A^{0}, H^{0}, h$ being the physical states of the theory and $G^{ \pm}, G^{0}$ the Goldstone bosons from the breaking of EW symmetry. Requiring that the scalar potential be bounded from below yields

$$
\lambda_{1}>0, \quad \lambda_{2}>0, \quad \lambda_{3}>-\sqrt{\lambda_{1} \lambda_{2}}, \quad \lambda_{3}+\lambda_{4}-\left|\lambda_{5}\right|>-\sqrt{\lambda_{1} \lambda_{2}} .
$$

Obtaining the correct EW vacuum as the minimum of the scalar potential (3.25) imposes the relations

$$
\begin{aligned}
& \mu_{1}^{2}=M^{2} s_{\beta}^{2}-\frac{v^{2}}{2}\left(\lambda_{1} c_{\beta}^{2}+\lambda_{345} s_{\beta}^{2}\right), \\
& \mu_{2}^{2}=M^{2} c_{\beta}^{2}-\frac{v^{2}}{2}\left(\lambda_{2} s_{\beta}^{2}+\lambda_{345} c_{\beta}^{2}\right),
\end{aligned}
$$

with $\lambda_{345} \equiv \lambda_{3}+\lambda_{4}+\lambda_{5}, s_{\beta} \equiv \sin \beta, c_{\beta} \equiv \cos \beta$ and $M^{2}=\mu^{2} / s_{\beta} c_{\beta}$. Similarly, the quartic couplings $\lambda_{1-5}$ in (3.25) may be re-expressed in terms of the masses of the physical states $m_{H^{ \pm}}, m_{A^{0}}, m_{H^{0}}, m_{h}$, the mixing angles $\alpha, \beta$ and $M^{2}$ as

$$
\begin{aligned}
& \lambda_{1}=\frac{1}{v^{2} c_{\beta}^{2}}\left(-M^{2} s_{\beta}^{2}+m_{h}^{2} s_{\alpha}^{2}+m_{H^{0}}^{2} c_{\alpha}^{2}\right), \\
& \lambda_{2}=\frac{1}{v^{2} s_{\beta}^{2}}\left(-M^{2} c_{\beta}^{2}+m_{h}^{2} c_{\alpha}^{2}+m_{H^{0}}^{2} s_{\alpha}^{2}\right), \\
& \lambda_{3}=\frac{1}{v^{2}}\left[-M^{2}+2 m_{H^{ \pm}}^{2}+\left(m_{H^{0}}^{2}-m_{h}^{2}\right) \frac{s_{2 \alpha}}{s_{2 \beta}}\right], \\
& \lambda_{4}=\frac{1}{v^{2}}\left(M^{2}+m_{A^{0}}^{2}-2 m_{H^{ \pm}}^{2}\right), \\
& \lambda_{5}=\frac{1}{v^{2}}\left(M^{2}-m_{A^{0}}^{2}\right) .
\end{aligned}
$$

We stress that since the masses of the physical states $m_{H^{ \pm}}, m_{A^{0}}, m_{H^{0}}, m_{h}$ and the mixing angles $\alpha, \beta$ are obtained upon EW symmetry breaking, the relations (A.4)-(A.8) only hold in the EW broken theory. 
We now perform an $\mathrm{SU}(2)$ rotation from the field basis $\Phi_{1}, \Phi_{2}$ to a field basis $H_{1}, H_{2}$ in which only $H_{1}$ takes a vev: $\left\langle H_{1}\right\rangle=\frac{v}{\sqrt{2}},\left\langle H_{2}\right\rangle=0$. This rotation is given by

$$
H_{1}=c_{\beta} \Phi_{1}+s_{\beta} \Phi_{2} \quad H_{2}=-s_{\beta} \Phi_{1}+c_{\beta} \Phi_{2}
$$

As seen from (A.1), for $\alpha=\beta-\pi / 2$, we obtain after the field rotation

$$
H_{1}=\Phi_{\mathrm{SM}}=\left(\begin{array}{c}
G^{+} \\
\frac{v+h+i G^{0}}{\sqrt{2}}
\end{array}\right), \quad H_{2}=\left(\begin{array}{c}
H^{+} \\
\frac{H^{0}+i A^{0}}{\sqrt{2}}
\end{array}\right),
$$

This is the alignment limit $c_{\beta-\alpha}=0$, in which $H_{1}$ corresponds exactly to the SM Higgs doublet. Away from this limit there is mixing of the neutral CP-even physical states $h, H^{0}$ between $H_{1,2}$.

The scalar potential for $H_{1,2}$ is given by

$$
\begin{aligned}
V_{\text {tree }}\left(H_{1}, H_{2}\right)= & \tilde{\mu}_{1}^{2}\left|H_{1}\right|^{2}+\tilde{\mu}_{2}^{2}\left|H_{2}\right|^{2}-\tilde{\mu}^{2}\left[H_{1}^{\dagger} H_{2}+\text { H.c. }\right]+\frac{\tilde{\lambda}_{1}}{2}\left|H_{1}\right|^{4} \\
& +\frac{\tilde{\lambda}_{2}}{2}\left|H_{2}\right|^{4}+\tilde{\lambda}_{3}\left|H_{1}\right|^{2}\left|H_{2}\right|^{2}+\tilde{\lambda}_{4}\left|H_{1}^{\dagger} H_{2}\right|^{2}+\frac{\tilde{\lambda}_{5}}{2}\left[\left(H_{1}^{\dagger} H_{2}\right)^{2}+\text { H.c. }\right] \\
& +\tilde{\lambda}_{6}\left[\left|H_{1}\right|^{2} H_{1}^{\dagger} H_{2}+\text { H.c. }\right]+\tilde{\lambda}_{7}\left[\left|H_{2}\right|^{2} H_{1}^{\dagger} H_{2}+\text { H.c. }\right] .
\end{aligned}
$$

The modified mass parameters $\tilde{\mu}_{1}^{2}, \tilde{\mu}_{2}^{2}, \tilde{\mu}^{2}$ and quartic couplings $\tilde{\lambda}_{1-5}$ are expressed in terms of $m_{H^{ \pm}}^{2}, m_{A^{0}}^{2}, m_{H^{0}}^{2}, m_{h}^{2}, M^{2}, \alpha$ and $\beta$ as

$$
\begin{aligned}
& \tilde{\mu}_{1}^{2}=-\frac{1}{2}\left[m_{H^{0}}^{2} c_{\beta-\alpha}^{2}+m_{h}^{2} s_{\beta-\alpha}^{2}\right]<0 \\
& \tilde{\mu}_{2}^{2}=M^{2}-\frac{1}{2}\left[\left(m_{H^{0}}^{2}-m_{h}^{2}\right) \frac{s_{2 \alpha}}{s_{2 \beta}}+m_{H^{0}}^{2} s_{\beta-\alpha}^{2}+m_{h}^{2} c_{\beta-\alpha}^{2}\right] \\
& \tilde{\mu}^{2}=-\frac{1}{2}\left(m_{H^{0}}^{2}-m_{h}^{2}\right) s_{2(\beta-\alpha)} \\
& \tilde{\lambda}_{1}=-\frac{\tilde{\mu}_{1}^{2}}{2 v^{2}}>0 \\
& \tilde{\lambda}_{2}=\frac{\left[-M^{2}\left(t_{\beta}^{2}+t_{\beta}^{-2}-2\right)+m_{H^{0}}^{2}\left(-s_{\beta-\alpha}^{2}+c_{\alpha}^{2} t_{\beta}^{2}+s_{\alpha}^{2} t_{\beta}^{-2}\right)+m_{h}^{2}\left(-c_{\beta-\alpha}^{2}+s_{\alpha}^{2} t_{\beta}^{2}+c_{\alpha}^{2} t_{\beta}^{-2}\right)\right]}{v^{2}} \\
& \tilde{\lambda}_{3}=\frac{\left[-2 M^{2}+2 m_{H^{ \pm}}^{2}+\left(m_{H^{0}}^{2}-m_{h}^{2}\right) \frac{s_{2 \alpha}}{s_{2 \beta}}+m_{H^{0}}^{2} s_{\beta-\alpha}^{2}+m_{h}^{2} c_{\beta-\alpha}^{2}\right]}{\left.v^{2}\right]} \\
& \tilde{\lambda}_{4}=\frac{\left[m_{A^{0}}^{2}-2 m_{H^{ \pm}}^{2}+m_{H^{0}}^{2} s_{\beta-\alpha}^{2}+m_{h}^{2} c_{\beta-\alpha}^{2}\right]}{v^{2}} \\
& \tilde{\lambda}_{5}=\frac{\left[-m_{A^{0}}^{2}+m_{H^{0}}^{2} s_{\beta-\alpha}^{2}+m_{h}^{2} c_{\beta-\alpha}^{2}\right]}{v^{2}} \\
& \tilde{\lambda}_{6}=\frac{\tilde{\mu}^{2}}{v^{2}} \\
& \tilde{\lambda}_{7}=\frac{s_{2 \beta}\left[M^{2}\left(t_{\beta}^{2}-t_{\beta}^{-2}\right)+m_{H^{0}}^{2}\left(\frac{c_{\alpha} t_{\beta}}{c_{\beta}}-\frac{s_{\alpha} t_{\beta}^{-1}}{s_{\beta}}\right) s_{\beta-\alpha}+m_{h}^{2}\left(\frac{c_{\alpha} t_{\beta}^{-1}}{s_{\beta}}-\frac{s_{\alpha} t_{\beta}}{c_{\beta}}\right) c_{\beta-\alpha}\right]}{2 v^{2}}
\end{aligned}
$$


The relations $\tilde{\mu}_{1}^{2}=-2 \tilde{\lambda}_{1} v^{2}$ and $\tilde{\mu}^{2}=\tilde{\lambda}_{6} v^{2}$ are necessary to obtain correct vev for $H_{1,2}$ upon minimization of (A.11). The relations among the masses of the new scalar states may be written in terms of $x_{0} \equiv m_{H^{0}}^{2} / m_{H^{ \pm}}^{2}$ and $x_{A} \equiv m_{A^{0}}^{2} / m_{H^{ \pm}}^{2}$ as

$$
\begin{aligned}
1-\frac{m_{A^{0}}^{2}}{m_{H^{ \pm}}^{2}} & \equiv 1-x_{A}=\frac{v^{2}}{2 m_{H^{ \pm}}^{2}}\left(\tilde{\lambda}_{5}-\tilde{\lambda}_{4}\right) \\
1-\frac{m_{H^{0}}^{2}}{m_{H^{ \pm}}^{2}} & \equiv 1-x_{0}=-\frac{v^{2}}{2 m_{H^{ \pm}}^{2}}\left(\tilde{\lambda}_{5}+\tilde{\lambda}_{4}\right)+c_{\beta-\alpha}^{2}\left[\frac{m_{h}^{2}}{m_{H^{ \pm}}^{2}}-x_{0}\right]
\end{aligned}
$$

In the alignment limit, the above expressions (A.12)-(A.13) simplify considerably, yielding

$$
\begin{aligned}
-\tilde{\mu}_{1}^{2} & =\frac{m_{h}^{2}}{2} \quad, \quad \tilde{\mu}_{2}^{2}=M^{2}-\frac{m_{h}^{2}}{2} \quad, \quad \tilde{\mu}^{2}=0 \\
\tilde{\lambda}_{1} & =\frac{m_{h}^{2}}{4 v^{2}} \quad, \quad \tilde{\lambda}_{2}=\frac{-M^{2}\left(t_{\beta}^{2}+t_{\beta}^{-2}-2\right)+m_{H^{0}}^{2}\left(-1+s_{\beta}^{2} t_{\beta}^{2}+c_{\beta}^{2} t_{\beta}^{-2}\right)+m_{h}^{2}}{v^{2}} \\
\tilde{\lambda}_{3} & =\frac{-2 M^{2}+2 m_{H^{ \pm}}^{2}+m_{h}^{2}}{v^{2}}, \quad \tilde{\lambda}_{4}=\frac{m_{H^{0}}^{2}+m_{A^{0}}^{2}-2 m_{H^{ \pm}}^{2}}{v^{2}}, \quad \tilde{\lambda}_{5}=\frac{m_{H^{0}}^{2}-m_{A^{0}}^{2}}{v^{2}} \\
\tilde{\lambda}_{6} & =0 \quad, \quad \tilde{\lambda}_{7}=s_{2 \beta}\left(t_{\beta}^{2}-t_{\beta}^{-2}\right) \frac{M^{2}-m_{H^{0}}^{2}}{2 v^{2}} .
\end{aligned}
$$

The interaction vertices $g_{h H^{+} H^{-}}, g_{h A^{0} A^{0}}$ and $g_{h H^{0} H^{0}}$ are in this limit proportional respectively to $\tilde{\lambda}_{3}, \tilde{\lambda}_{3}+\tilde{\lambda}_{4}-\tilde{\lambda}_{5}$ and $\tilde{\lambda}_{3}+\tilde{\lambda}_{4}+\tilde{\lambda}_{5}$.

\section{A.2 Electroweak precision observables}

We turn now to the discussion of EW precision constraints in the 2HDM. The non-SM contributions to the oblique parameters $S$ and $T$ are given by (3.50)-(3.51) (the general expressions for the various oblique parameters in models with an arbitrary number of scalar doublets may be found in [118]), with the functions $F_{A, B}, G_{A, B, C}, \hat{G}_{A, B}$ being

$$
\begin{aligned}
F_{A, B}= & \frac{m_{A}^{2}+m_{B}^{2}}{2}-\frac{m_{A}^{2} m_{B}^{2}}{m_{A}^{2}-m_{B}^{2}} \log \left(\frac{m_{A}^{2}}{m_{B}^{2}}\right) \\
G_{A, B, C}= & -\frac{16}{3}+5 \frac{m_{A}^{2}+m_{B}^{2}}{m_{C}^{2}}-2 \frac{\left(m_{A}^{2}-m_{B}^{2}\right)^{2}}{m_{C}^{4}} \\
& +\frac{3}{m_{C}^{2}}\left[\frac{m_{A}^{4}+m_{B}^{4}}{m_{A}^{2}-m_{B}^{2}}+\frac{m_{A}^{4}-m_{B}^{4}}{m_{C}^{2}}+\frac{\left(m_{A}^{2}-m_{B}^{2}\right)^{3}}{3 m_{C}^{4}}\right] \log \left(\frac{m_{A}^{2}}{m_{B}^{2}}\right) \\
& +\frac{m_{C}^{4}+m_{C}^{2}\left(m_{A}^{2}+m_{B}^{2}\right)+\left(m_{A}^{2}-m_{B}^{2}\right)^{2}}{m_{C}^{6}} f\left(r_{A, B, C}, t_{A, B, C}\right) \\
\hat{G}_{A, B}= & G_{A, B, B}-24+12\left(\frac{m_{A}^{2}-m_{B}^{2}}{m_{B}^{2}}-\frac{m_{A}^{2}+m_{B}^{2}}{m_{A}^{2}-m_{B}^{2}}\right) \log \left(\frac{m_{A}^{2}}{m_{B}^{2}}\right)+12 \frac{f\left(r_{A, B, B}, t_{A, B, B}\right)}{m_{B}^{2}}
\end{aligned}
$$

with

$$
\begin{aligned}
& r_{A, B, C}=m_{C}^{4}+m_{C}^{2}\left(m_{A}^{2}+m_{B}^{2}\right)+\left(m_{A}^{2}-m_{B}^{2}\right)^{2} \\
& t_{A, B, C}=m_{A}^{2}+m_{B}^{2}-m_{C}^{2}
\end{aligned}
$$




$$
f(r, t)=\left\{\begin{array}{cc}
\sqrt{r} \log \left(\left|\frac{t-\sqrt{r}}{t+\sqrt{r}}\right|\right) & r>0 \\
0 & r=0 \\
2 \sqrt{-r} \arctan \left(\frac{\sqrt{-r}}{t}\right) & r<0
\end{array}\right.
$$

Finally, we stress that the potential (A.11) preserves custodial symmetry in the limit $\tilde{\lambda}_{4}=$ $\tilde{\lambda}_{5}$ (see e.g. [148]), which from (A.14) corresponds to $m_{A^{0}}=m_{H^{ \pm}}$. To see this, instead of expressing the $2 \mathrm{HDM}$ scalar potential in terms of $H_{1,2}$, we can introduce the $2 \times 2$ matrices $\mathbf{\Phi}_{1}=\left(i \sigma_{2} H_{1}^{*}, H_{1}\right), \mathbf{\Phi}_{2}=\left(i \sigma_{2} H_{2}^{*}, H_{2}\right)$. The scalar potential for the $2 \mathrm{HDM}$ then reads

$$
\begin{aligned}
& V=-\frac{\tilde{\mu}_{1}^{2}}{2} \operatorname{Tr}\left[\boldsymbol{\Phi}_{1}^{\dagger} \mathbf{\Phi}_{1}\right]+\frac{\tilde{\mu}_{2}^{2}}{2} \operatorname{Tr}\left[\boldsymbol{\Phi}_{2}^{\dagger} \mathbf{\Phi}_{2}\right]+\frac{\tilde{\mu}^{2}}{2}\left(\operatorname{Tr}\left[\boldsymbol{\Phi}_{1}^{\dagger} \mathbf{\Phi}_{2}\right]+\operatorname{Tr}\left[\boldsymbol{\Phi}_{2}^{\dagger} \mathbf{\Phi}_{1}\right]\right) \\
& +\frac{\tilde{\lambda}_{1}}{4}\left(\operatorname{Tr}\left[\boldsymbol{\Phi}_{1}^{\dagger} \mathbf{\Phi}_{1}\right]\right)^{2}+\frac{\tilde{\lambda}_{2}}{4}\left(\operatorname{Tr}\left[\boldsymbol{\Phi}_{2}^{\dagger} \mathbf{\Phi}_{2}\right]\right)^{2}+\frac{\tilde{\lambda}_{3}}{4} \operatorname{Tr}\left[\boldsymbol{\Phi}_{1}^{\dagger} \boldsymbol{\Phi}_{1}\right] \operatorname{Tr}\left[\boldsymbol{\Phi}_{2}^{\dagger} \mathbf{\Phi}_{2}\right] \\
& +\frac{\tilde{\lambda}_{4}+\tilde{\lambda}_{5}}{16}\left(\operatorname{Tr}\left[\boldsymbol{\Phi}_{1}^{\dagger} \mathbf{\Phi}_{2}\right]+\operatorname{Tr}\left[\boldsymbol{\Phi}_{2}^{\dagger} \boldsymbol{\Phi}_{1}\right]\right)^{2}-\frac{\tilde{\lambda}_{4}-\tilde{\lambda}_{5}}{16}\left(\operatorname{Tr}\left[\boldsymbol{\Phi}_{1}^{\dagger} \boldsymbol{\Phi}_{2} \sigma_{3}\right]-\operatorname{Tr}\left[\sigma_{3} \boldsymbol{\Phi}_{2}^{\dagger} \boldsymbol{\Phi}_{1}\right]\right)^{2} \\
& +\frac{1}{16}\left(\tilde{\lambda}_{6} \operatorname{Tr}\left[\boldsymbol{\Phi}_{1}^{\dagger} \boldsymbol{\Phi}_{1}\right]+\tilde{\lambda}_{7} \operatorname{Tr}\left[\boldsymbol{\Phi}_{2}^{\dagger} \boldsymbol{\Phi}_{2}\right]\right)\left(\operatorname{Tr}\left[\boldsymbol{\Phi}_{1}^{\dagger} \boldsymbol{\Phi}_{2}\right]+\operatorname{Tr}\left[\boldsymbol{\Phi}_{2}^{\dagger} \boldsymbol{\Phi}_{1}\right]\right)
\end{aligned}
$$

Both $\boldsymbol{\Phi}_{1,2}$ transform as bi-doublets of a global symmetry $\mathrm{SU}(2)_{L} \times \mathrm{SU}(2)_{R}: \boldsymbol{\Phi}_{i} \rightarrow L \boldsymbol{\Phi}_{i} R$ (with $L \in \mathrm{SU}(2)_{L}$ and $R \in \mathrm{SU}(2)_{R}$ ). The potential (A.20) is then invariant under a custodial $\mathrm{SU}(2)_{L} \times \mathrm{SU}(2)_{R}$ in the absence of the term proportional to $\tilde{\lambda}_{4}-\tilde{\lambda}_{5}$.

\section{B Effective theory matching in the EW broken phase}

We now relate the Wilson coefficients from the $D=6$ operators for the $\mathrm{SM} \mathrm{SU}(2)_{L} \times \mathrm{U}(1)_{Y}$ invariant effective field theory given in (2.2), (2.5) and (2.6) to the SM effective Lagrangian after EW symmetry breaking. The complete set of relations from the full $D \leq 6$ effective Lagrangian (2.1) may be found in [26]. In the following we present the generic relations for the 3- and 4-point interactions involving Higgs/gauge bosons. The CP-conserving 3-point interactions involving at least one light Higgs scalar $h$ are obtained from $\mathcal{L}_{\text {SILH }}$ and read

$$
\begin{aligned}
\mathcal{L}_{3 h}= & -\frac{m_{H}^{2} g_{h h h}^{(1)}}{2 v} h^{3}+\frac{g_{h h h}^{(2)}}{2} h \partial_{\mu} h \partial^{\mu} h-\frac{g_{h g g}}{4} G_{\mu \nu}^{a} G_{a}^{\mu \nu} h-\frac{g_{h \gamma \gamma}}{4} F_{\mu \nu} F^{\mu \nu} h \\
& -\frac{g_{h w w}^{(1)}}{2} W^{\mu \nu} W_{\mu \nu}^{\dagger} h-\left[g_{h w w}^{(2)} W^{\nu} \partial^{\mu} W_{\mu \nu}^{\dagger} h+\text { h.c. }\right]+g_{h w w}^{(3)} W_{\mu}^{\dagger} W^{\mu} h \\
& -\frac{g_{h z z}^{(1)}}{4} Z_{\mu \nu} Z^{\mu \nu} h-g_{h z z}^{(2)} Z_{\nu} \partial_{\mu} Z^{\mu \nu} h+\frac{g_{h z z}^{(3)}}{2} Z_{\mu} Z^{\mu} h \\
& -\frac{g_{h a z}^{(1)}}{2} Z_{\mu \nu} F^{\mu \nu} h-g_{h a z}^{(2)} Z_{\nu} \partial_{\mu} F^{\mu \nu} h .
\end{aligned}
$$

where we have introduced abelian field-strength tensors $W_{\mu \nu}, Z_{\mu \nu}$ and $F_{\mu \nu}$ for the $W$-boson, $Z$-boson and photon respectively, and $G_{\mu \nu}^{a}$ is still the non-abelian gluon field-strength tensor. The CP-conserving 4-point interactions involving at least one light Higgs scalar $h$ 
similarly read

$$
\begin{aligned}
\mathcal{L}_{4 h}= & -\frac{m_{H}^{2} g_{h h h h}^{(1)}}{8 v^{2}} h^{4}+\frac{g_{h h h h}^{(2)}}{2} h^{2} \partial_{\mu} h \partial^{\mu} h-\frac{g_{h h g g}}{8} G_{\mu \nu}^{a} G_{a}^{\mu \nu} h^{2}-\frac{g_{h h \gamma \gamma}}{8} F_{\mu \nu} F^{\mu \nu} h^{2} \\
& -\frac{g_{h h w w}^{(1)}}{4} W^{\mu \nu} W_{\mu \nu}^{\dagger} h^{2}-\frac{1}{2}\left[g_{h h w w}^{(2)} W^{\nu} \partial^{\mu} W_{\mu \nu}^{\dagger} h^{2}+\text { h.c. }\right]+\frac{g_{h h w w}^{(3)}}{2} W_{\mu}^{\dagger} W^{\mu} h^{2} \\
& -\frac{g_{h h z z}^{(1)}}{8} Z_{\mu \nu} Z^{\mu \nu} h^{2}-\frac{g_{h h z}^{(2)}}{2} Z_{\nu} \partial_{\mu} Z^{\mu \nu} h^{2}+\frac{g_{h h z z}^{(3)}}{4} Z_{\mu} Z^{\mu} h^{2}-\frac{g_{h h a z}^{(1)}}{4} Z_{\mu \nu} F^{\mu \nu} h^{2} \\
& -\frac{g_{h h a z}^{(2)}}{2} Z_{\nu} \partial_{\mu} F^{\mu \nu} h^{2}-i g_{h a w w}^{(1)} F^{\mu \nu} W_{\mu} W_{\nu}^{\dagger} h+\left[i g_{h a w w}^{(2)} W^{\mu \nu} A_{\mu} W_{\nu}^{\dagger} h+\text { h.c. }\right] \\
& +i g_{h a w w}^{(3)} A_{\mu} W_{\nu} W_{\rho}^{\dagger}\left[\eta^{\mu \rho} \partial^{\nu} h-\eta^{\mu \nu} \partial^{\rho} h\right]-i g_{h z w w}^{(1)} Z^{\mu \nu} W_{\mu} W_{\nu}^{\dagger} h \\
& +\left[i g_{h z w w}^{(2)} W^{\mu \nu} Z_{\mu} W_{\nu}^{\dagger} h+\text { h.c. }\right]-i g_{h z w w}^{(3)} Z_{\mu} W_{\nu} W_{\rho}^{\dagger}\left[\eta^{\mu \rho} \partial^{\nu} h-\eta^{\mu \nu} \partial^{\rho} h\right] .
\end{aligned}
$$

We note that the 4-point interactions involving one Higgs $h$ and three gluons (relevant, e.g. for Higgs production in association with a jet via gluon fusion) are already taken into account by the $g_{h g g}$ term in (B.1). In a similar fashion to (B.1) and (B.2), the CP-odd 3- and 4point interactions involving at least one light Higgs scalar $h$ are obtained from $\mathcal{L}_{\mathrm{CP}}$, yielding

$$
\begin{aligned}
\tilde{\mathcal{L}}_{h}= & -\frac{\tilde{g}_{h g g}}{4} G_{\mu \nu}^{a} \tilde{G}^{\mu \nu} h-\frac{\tilde{g}_{h \gamma \gamma}}{4} F_{\mu \nu} \tilde{F}^{\mu \nu} h-\frac{\tilde{g}_{h w w}}{2} W^{\mu \nu} \tilde{W}_{\mu \nu}^{\dagger} h-\frac{\tilde{g}_{h z z}}{4} Z_{\mu \nu} \tilde{Z}^{\mu \nu} h-\frac{\tilde{g}_{h a z}}{2} Z_{\mu \nu} \tilde{F}^{\mu \nu} h \\
& -\frac{\tilde{g}_{h h g g}}{8} G_{\mu \nu}^{a} \tilde{G}_{a}^{\mu \nu} h^{2}-\frac{\tilde{g}_{h h \gamma \gamma}}{8} F_{\mu \nu} \tilde{F}^{\mu \nu} h^{2}-\frac{\tilde{g}_{h h w w}}{4} W^{\mu \nu} \tilde{W}_{\mu \nu}^{\dagger} h^{2}-\frac{\tilde{g}_{h h z z}}{8} Z_{\mu \nu} \tilde{Z}^{\mu \nu} h^{2} \\
& -\frac{\tilde{g}_{h h a z}}{4} Z_{\mu \nu} \tilde{F}^{\mu \nu} h^{2}+i \tilde{g}_{h a w w}^{(1)} \tilde{F}^{\mu \nu} W_{\mu} W_{\nu}^{\dagger} h+\left[i \tilde{g}_{h a w w}^{(2)} \tilde{W}^{\mu \nu} A_{\mu} W_{\nu}^{\dagger} h+\text { h.c. }\right] \\
& +i \tilde{g}_{h z w w}^{(1)} \tilde{Z}^{\mu \nu} W_{\mu} W_{\nu}^{\dagger} h-\left[i \tilde{g}_{h z w w}^{(2)} \tilde{W}^{\mu \nu} Z_{\mu} W_{\nu}^{\dagger} h+\text { h.c. }\right] .
\end{aligned}
$$

Finally, the 3- and 4-point gauge boson self-interactions receive CP-even contributions from both $\mathcal{L}_{\mathrm{SILH}}$ and $\mathcal{L}_{G}$ (they also receive CP-odd contributions from $\mathcal{L}_{\mathrm{CP}}$, which we do not include here). We adopt here the parametrization of triple gauge couplings (TGCs) from [10, 149], which yields

$$
\begin{aligned}
\mathcal{L}_{3 V}= & e g_{1}^{\gamma}\left[i W_{\mu \nu}^{\dagger} A^{\mu} W^{\nu}+\text { h.c. }\right]+g c_{W} g_{1}^{Z}\left[i W_{\mu \nu}^{\dagger} Z^{\mu} W^{\nu}+\text { h.c. }\right] \\
& +e \kappa_{\gamma}\left(i F_{\mu \nu} W^{\mu} W^{\nu \dagger}\right)+g c_{W} \kappa_{Z}\left(i Z_{\mu \nu} W^{\mu} W^{\nu \dagger}\right) \\
& +\frac{e \lambda_{\gamma}}{m_{W}^{2}} i W_{\mu \nu} W_{\nu \rho}^{\dagger} F_{\rho \mu}+\frac{g c_{W} \lambda_{Z}}{m_{W}^{2}} i W_{\mu \nu} W_{\nu \rho}^{\dagger} Z_{\rho \mu},
\end{aligned}
$$

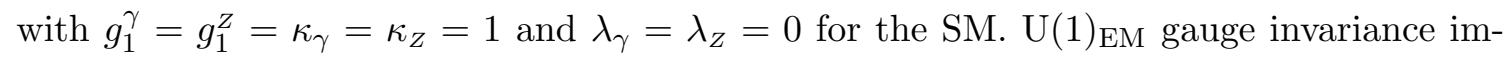
poses $g_{1}^{\gamma}=1$, while the (spontaneously broken) $\mathrm{SU}(2)_{L} \times \mathrm{U}(1)_{Y}$ gauge invariance combined with the restriction to $D \leq 6$ effective operators in $\mathcal{L}_{\text {Eff }}$ lead to the relations [10]

$$
g_{1}^{Z}=\kappa_{Z}+\frac{s_{W}^{2}}{c_{W}^{2}}\left(\kappa_{\gamma}-1\right), \quad \lambda_{\gamma}=\lambda_{Z}
$$

These degeneracies imply that there can be at most three independent contributions to anomalous TCGs from $\mathcal{L}_{\mathrm{SILH}}$ and $\mathcal{L}_{G}$. The operators proportional to $g_{1}^{Z}, \kappa_{Z}$ and $\kappa_{\gamma}$ 
$\mathcal{L}_{3 h}$ couplings vs. $\mathrm{SU}(2)_{L} \times \mathrm{U}(1)_{Y}(D \leq 6)$ Wilson coefficients

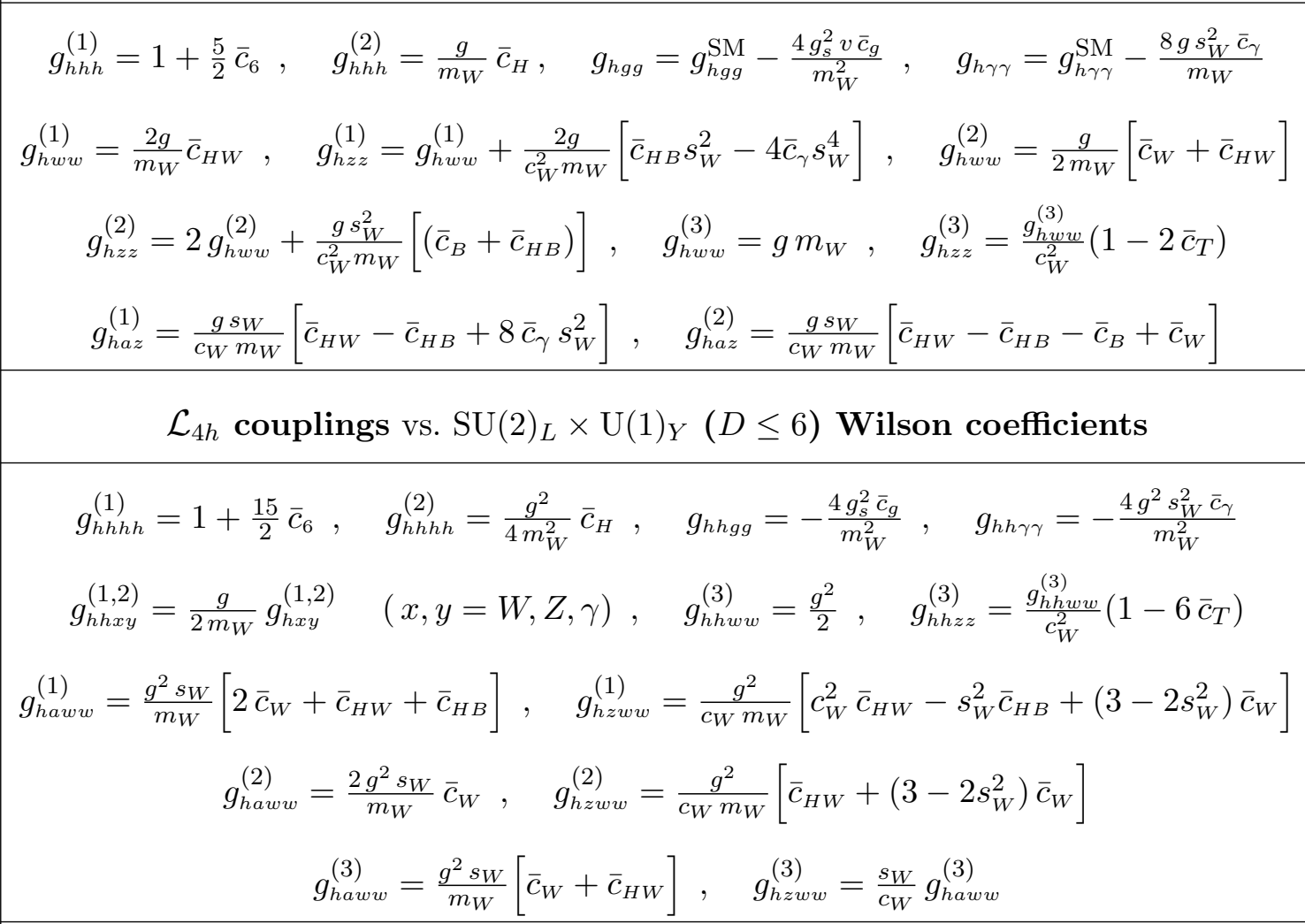

$\tilde{\mathcal{L}}_{h}$ couplings vs. $\mathrm{SU}(2)_{L} \times \mathrm{U}(1)_{Y}(D \leq 6)$ Wilson coefficients

$$
\begin{gathered}
\tilde{g}_{h g g}=-\frac{4 g_{s}^{2} v \tilde{c}_{g}}{m_{W}^{2}}, \quad \tilde{g}_{h \gamma \gamma}=-\frac{8 g s_{W}^{2} \tilde{c}_{\gamma}}{m_{W}}, \quad \tilde{g}_{h h g g}=-\frac{4 g_{s}^{2} \tilde{c}_{g}}{m_{W}^{2}}, \quad \tilde{g}_{h h \gamma \gamma}=-\frac{4 g^{2} s_{W}^{2} \tilde{c}_{\gamma}}{m_{W}^{2}} \\
\tilde{g}_{h w w}=\frac{2 g}{m_{W}} \tilde{c}_{H W}, \quad \tilde{g}_{h z z}=\tilde{g}_{h w w}+\frac{2 g}{c_{W}^{2} m_{W}}\left[\tilde{c}_{H B} s_{W}^{2}-4 \tilde{c}_{\gamma} s_{W}^{4}\right] \\
\tilde{g}_{h h x y}=\frac{g}{2 m_{W}} g_{h x y} \quad(x, y=W, Z, \gamma), \quad \tilde{g}_{h a w w}^{(1)}=\frac{g^{2} s_{W}}{m_{W}}\left[\tilde{c}_{H W}-\tilde{c}_{H B}\right] \\
\tilde{g}_{h z w w}^{(1)}=\frac{g^{2}}{c_{W} m_{W}}\left[\left(2-s_{W}^{2}\right) \tilde{c}_{H W}+s_{W}^{2} \tilde{c}_{H B}\right], \quad g_{h z w w}^{(2)}=\frac{g^{2}}{c_{W} m_{W}}\left[\bar{c}_{H W}+\left(3-2 s_{W}^{2}\right) \bar{c}_{W}\right] \\
\tilde{g}_{h a w w}^{(2)}=\frac{g^{2} s_{W}}{m_{W}} \tilde{c}_{H W}, \quad \tilde{g}_{h z w w}^{(2)}=\frac{2 c_{W}}{s_{W}} \tilde{g}_{h a w w}^{(2)}, \quad \tilde{g}_{h a z}=\frac{g s_{W}}{c_{W} m_{W}}\left[\tilde{c}_{H W}-\tilde{c}_{H B}+8 \tilde{c}_{\gamma} s_{W}^{2}\right]
\end{gathered}
$$

Table 5. Relations between the different couplings appearing in $\mathcal{L}_{3 h}, \mathcal{L}_{4 h}, \tilde{\mathcal{L}}_{h}$ and the Wilson coefficients for the $D \leq 6$ effective operators in $\mathcal{L}_{\text {Eff. }}$. 


\begin{tabular}{c}
$\mathcal{L}_{3 V}$ couplings vs. SU $(2)_{L} \times \mathrm{U}(1)_{Y}(D \leq 6)$ Wilson coefficients \\
\hline$g_{1}^{Z}=1-\frac{1}{c_{W}^{2}}\left[\bar{c}_{H W}-\left(2 s_{W}^{2}-3\right) \bar{c}_{W}\right], \quad \kappa_{Z}=1-\frac{1}{c_{W}^{2}}\left[c_{W}^{2} \bar{c}_{H W}-s_{W}^{2} \bar{c}_{H B}-\left(2 s_{W}^{2}-3\right) \bar{c}_{W}\right]$ \\
$g_{1}^{\gamma}=1, \quad \kappa_{\gamma}=1-2 \bar{c}_{W}-\bar{c}_{H W}-\bar{c}_{H B}, \quad \lambda_{\gamma}=\lambda_{Z}=3 g^{2} \bar{c}_{3 W}$ \\
\hline $\mathcal{L}_{4 V}$ couplings vs. SU $(2)_{L} \times \mathrm{U}(1)_{Y}(D \leq 6)$ Wilson coefficients \\
$g_{2}^{W}=1-2 \bar{c}_{H W}-4 \bar{c}_{W}, \quad g_{2}^{Z}=1-\frac{1}{c_{W}^{2}}\left[2 \bar{c}_{H W}+2\left(2-s_{W}^{2}\right) \bar{c}_{W}\right]$ \\
$g_{2}^{\gamma}=1, \quad g_{2}^{\gamma Z}=1-\frac{1}{c_{W}^{2}}\left[\bar{c}_{H W}+\left(3-2 s_{W}^{2}\right) \bar{c}_{W}\right]$ \\
$\lambda_{W}=\lambda_{\gamma W}=\lambda_{\gamma Z}=\lambda_{W Z}=6 g^{2} \bar{c}_{3 W}$ \\
\hline
\end{tabular}

Table 6. The Trilinear and Quartic Gauge Couplings appearing in $\mathcal{L}_{3 V}$ and $\mathcal{L}_{4 V}$ as a function of the Wilson coefficients for the $D \leq 6$ effective operators in $\mathcal{L}_{\text {Eff }}$.

in (B.4) contain one derivative and are obtained from $\mathcal{L}_{\mathrm{SILH}}$, which then gives rise at most to two independent contributions to anomalous TGCs. The last two operators in (B.4) contain three derivatives and are obtained from $\mathcal{L}_{G}$, which then gives rise to a sole linearly independent contribution to anomalous TGCs. This is clear for the operator $\bar{c}_{3 W} \mathcal{O}_{3 W}$, as it is constructed from three gauge field-strengths and only their abelian parts can contribute to TGCs (after EW symmetry breaking it directly leads to the last two operators in (B.4) with $\lambda_{\gamma}=\lambda_{Z}$ ). The operator $\bar{c}_{2 W} \mathcal{O}_{D W}$ also leads after EW symmetry breaking to those operators with $\lambda_{\gamma}=\lambda_{z}$, but also produces operators of the form

$$
g \epsilon_{i j k}\left(\square W_{\mu \nu}^{i}\right) W_{\mu}^{j} W_{\nu}^{k}
$$

These operators however either vanish or reduce to operators from (B.4) for on-shell gauge bosons (as well as for the virtual photon). Therefore, the parametrization of TGCs (B.4) holds. Moreover, we can safely disregard the contribution of $\bar{c}_{2 W}$ to $\lambda_{\gamma}=\lambda_{Z}$ in the following discussion, since this operator can be re-casted as (very constrained) fermionic currents and the operators involving the Higgs we have already considered [150]. For the case of quartic gauge couplings (QGCs) we have

$$
\begin{aligned}
\mathcal{L}_{4 V}= & \frac{g^{2} g_{2}^{W}}{2}\left[W_{\mu} W^{\mu} W_{\nu}^{\dagger} W^{\nu \dagger}-W^{\mu} W_{\mu}^{\dagger} W^{\nu} W_{\nu}^{\dagger}\right]+e^{2} g_{2}^{\gamma}\left[A_{\mu} A^{\nu} W_{\nu}^{\dagger} W^{\mu}-A^{\mu} A_{\mu} W^{\nu} W_{\nu}^{\dagger}\right] \\
& +2 e g c_{W} g_{2}^{\gamma Z}\left[A_{\mu} Z^{\nu} W_{\nu}^{\dagger} W^{\mu}-A^{\mu} Z_{\mu} W^{\nu} W_{\nu}^{\dagger}\right]+g^{2} c_{W}^{2} g_{2}^{Z}\left[Z_{\mu} Z^{\nu} W_{\nu}^{\dagger} W^{\mu}-Z^{\mu} Z_{\mu} W^{\nu} W_{\nu}^{\dagger}\right] \\
& +\frac{e g c_{W} \lambda_{\gamma z}}{m_{W}^{2}}\left[W_{\mu} W^{\mu \rho \dagger}\left(A_{\nu} Z^{\nu \rho}+Z_{\nu} F^{\nu \rho}\right)-W_{\mu} W^{\nu \rho \dagger}\left(A_{\nu} Z^{\mu \rho}+Z_{\nu} F^{\mu \rho}\right)\right] \\
& +\frac{g^{2} \lambda_{W}}{2 m_{W}^{2}}\left[W_{\mu} W^{\mu \rho \dagger} W_{\nu}^{\dagger} W^{\nu \rho}-W_{\mu} W^{\nu \rho \dagger} W_{\nu}^{\dagger} W^{\mu \rho}\right] \\
& +\frac{e^{2} \lambda_{\gamma W}}{m_{W}^{2}}\left[W_{\mu} W^{\mu \rho \dagger} A_{\nu} F^{\nu \rho}-W_{\mu} W^{\nu \rho \dagger} A_{\nu} F^{\mu \rho}\right]
\end{aligned}
$$



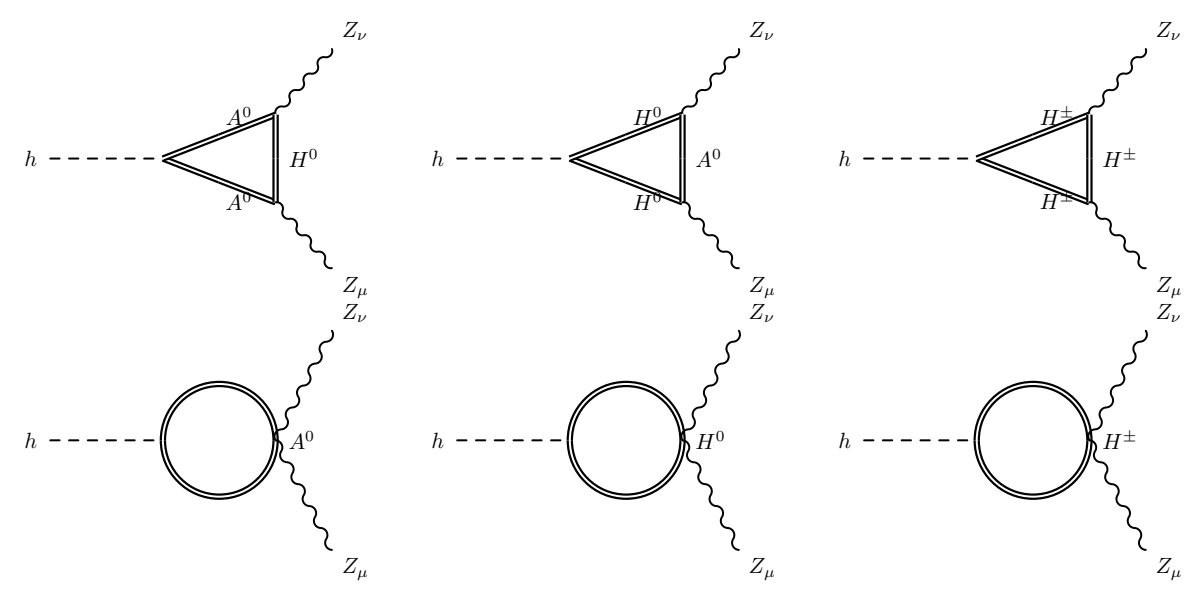

Figure 10. 1-loop Feynman diagrams involving the $H^{ \pm}, A^{0}, H^{0}$ scalars and contributing to the $Z_{\mu} Z_{\nu} h$ vertex in the alignment limit $c_{\beta-\alpha}=0$.

$$
+\frac{g^{2} c_{W}^{2} \lambda_{W Z}}{m_{W}^{2}}\left[W_{\mu} W^{\mu \rho \dagger} Z_{\nu} Z^{\nu \rho}-W_{\mu} W^{\nu \rho \dagger} Z_{\nu} Z^{\mu \rho}\right]
$$

where again $g_{2}^{W}=g_{2}^{\gamma}=g_{2}^{Z}=g_{2}^{\gamma Z}=1$ and $\lambda_{W}=\lambda_{\gamma W}=\lambda_{\gamma Z}=\lambda_{W Z}=0$ corresponds to the SM case.

The correspondence between the Wilson coefficients $\bar{c}_{i}$ for the effective operators from (2.2), (2.5), (2.6) and the various couplings arising after EW symmetry breaking in (B.1)-(B.4), (B.7) is shown in tables 5 and 6. In the next section, we compute the values of these couplings by direct matching in the broken EW theory, for the specific setup of Benchmark $\mathrm{A}$ in 3.2.1: the 2HDM in the alignment limit $c_{\beta-\alpha}=0$.

\section{B.1 A specific example: 2HDM in the alignment limit $c_{\beta-\alpha}=0$}

Here we will obtain the values of the various couplings from (B.1) for the 2HDM in the alignment limit $c_{\beta-\alpha}=0$, by direct matching in the broken $\mathrm{EW}$ theory. We focus on the $V_{\mu} V_{\nu} h$ interaction vertices, which receive contributions from loops of scalars $H^{ \pm}, A^{0}, H^{0}$ (these are shown in figure 10 for the case $V_{\mu}=Z_{\mu}$ ). We note that loops involving only SM particles cancel in the matching between full and effective theories and may then be disregarded, while loops involving both SM particles and $H^{ \pm}, A^{0}, H^{0}$ scalars vanish in the alignment limit.

We compute the 1-loop contributions to $V_{\mu} V_{\nu} h$ and expand the result in powers of the 4-momenta of the $Z$-bosons $p_{1}, p_{2}$. By truncating the expansion at order $\mathcal{O}\left(p^{2}\right)$, we obtain

$$
\Delta \mathcal{L}=\Delta \mathcal{L}_{W}+\Delta \mathcal{L}_{Z}+\Delta \mathcal{L}_{\gamma}
$$

with

$$
\begin{aligned}
\Delta \mathcal{L}_{W}= & -\frac{g_{h w w}^{(1)}}{2} W^{\mu \nu} W_{\mu \nu}^{\dagger} h-\left[g_{h w w}^{(2)} W^{\nu} \partial^{\mu} W_{\mu \nu}^{\dagger} h+\text { h.c. }\right]+g_{h w w}^{(3)} W^{\mu} W_{\mu}^{\dagger} h \\
& +g_{h w w}^{(4)}\left(\partial_{\mu} W_{\nu}^{\dagger}\right)\left(\partial^{\mu} W^{\nu}\right) h+\left[g_{h w w}^{(5)} W^{\nu} \partial^{\mu} \partial_{\mu} W_{\nu}^{\dagger} h+\text { h.c. }\right] \\
& +g_{h w w}^{(6)} \partial^{\mu} W_{\mu} \partial^{\nu} W_{\nu}^{\dagger} h
\end{aligned}
$$




$$
\begin{aligned}
\Delta \mathcal{L}_{Z}= & -\frac{g_{h z z}^{(1)}}{4} Z_{\mu \nu} Z^{\mu \nu} h-g_{h z z}^{(2)} Z_{\nu} \partial_{\mu} Z^{\mu \nu} h+\frac{g_{h z z}^{(3)}}{2} Z_{\mu} Z^{\mu} h \\
& +g_{h z z}^{(4)}\left(\partial_{\mu} Z_{\nu}\right)\left(\partial^{\mu} Z^{\nu}\right) h+g_{h z z}^{(5)} Z_{\nu} \partial_{\mu} \partial^{\mu} Z^{\nu} h+g_{h z z}^{(6)} \partial_{\mu} Z^{\mu} \partial_{\nu} Z^{\nu} h \\
\Delta \mathcal{L}_{\gamma}= & -\frac{g_{h \gamma \gamma}^{(1)}}{4} F_{\mu \nu} F^{\mu \nu} h-\frac{g_{h \gamma z}^{(1)}}{2} Z_{\mu \nu} F^{\mu \nu} h-g_{h \gamma z}^{(2)} Z_{\nu} \partial_{\mu} F^{\mu \nu} h
\end{aligned}
$$

with the last three terms in both (B.9) and (B.10) corresponding to e.o.m.-vanishing terms, which are nevertheless generated by the off-shell 1-loop corrections. Performing an expansion to linear order in $1-m_{H^{0}}^{2} / m_{H^{ \pm}}^{2}=1-x_{0}$ and $1-m_{A^{0}}^{2} / m_{H^{ \pm}}^{2}=1-x_{A}$ yields for $\Delta \mathcal{L}_{W}$

$$
\begin{aligned}
& g_{h w w}^{(1)}=\frac{-g^{2} v}{192 \pi^{2} m_{H^{ \pm}}^{2}}\left[\frac{g_{0}+g_{A}+2 g_{+}}{2}+\left(1-x_{0}\right) \frac{4 g_{0}+g_{+}}{10}+\left(1-x_{A}\right) \frac{4 g_{A}+g_{+}}{10}\right] \\
& g_{h w w}^{(2,6)}=\frac{g_{h w w}^{(4)}}{2}=\frac{2 g_{h w w}^{(5)}}{5}=\frac{g^{2} v}{192 \pi^{2} m_{H^{ \pm}}^{2}}\left[\left(1-x_{0}\right) \frac{g_{0}-g_{+}}{20}+\left(1-x_{A}\right) \frac{g_{A}-g_{+}}{20}\right] \\
& g_{h w w}^{(3)}=\frac{g^{2} v}{192 \pi^{2}}\left[\left(1-x_{0}\right)\left(g_{+}-g_{0}\right)+\left(1-x_{A}\right)\left(g_{+}-g_{A}\right)\right]
\end{aligned}
$$

with $g_{0} \equiv g_{h H^{0} H^{0}} / v, g_{A} \equiv g_{h A^{0} A^{0}} / v$ and $g_{+} \equiv g_{h H^{+} H^{-}} / v$, and the trilinear scalar couplings $g_{h H^{+} H^{-}}, g_{h H^{0} H^{0}}$ and $g_{h A^{0} A^{0}}$ given in the EW broken theory with $c_{\beta-\alpha}=0$ by

$$
\begin{aligned}
g_{h H^{+} H^{-}} & =\frac{\left(-2 M^{2}+2 m_{H^{ \pm}}^{2}+m_{h}^{2}\right)}{v}=\tilde{\lambda}_{3} v \\
g_{h H^{0} H^{0}} & =\frac{\left(-2 M^{2}+2 m_{H^{0}}^{2}+m_{h}^{2}\right)}{v}=\left(\tilde{\lambda}_{3}+\tilde{\lambda}_{4}+\tilde{\lambda}_{5}\right) v \\
g_{h A^{0} A^{0}} & =\frac{\left(-2 M^{2}+2 m_{A^{0}}^{2}+m_{h}^{2}\right)}{v}=\left(\tilde{\lambda}_{3}+\tilde{\lambda}_{4}-\tilde{\lambda}_{5}\right) v
\end{aligned}
$$

By means of (B.15), the relations (B.12)-(B.14) may be written as

$$
\begin{aligned}
g_{h w w}^{(1)} & =\frac{-g^{2} v}{192 \pi^{2} m_{H^{ \pm}}^{2}}\left[\left(2 \tilde{\lambda}_{3}+\tilde{\lambda}_{4}\right)+\mathcal{O}\left[\left(1-x_{0}\right),\left(1-x_{A}\right)\right]\right] \\
g_{h w w}^{(2,4-6)} & \sim \mathcal{O}\left[\left(1-x_{0}\right)^{2},\left(1-x_{A}\right)^{2}\right] \\
g_{h w w}^{(3)} & =\frac{g^{2} v}{192 \pi^{2}}\left[\left(1-x_{0}\right)\left(\tilde{\lambda}_{4}+\tilde{\lambda}_{5}\right)+\left(1-x_{A}\right)\left(\tilde{\lambda}_{4}-\tilde{\lambda}_{5}\right)\right]
\end{aligned}
$$

Performing a similar expansion in $\Delta \mathcal{L}_{Z}$ then yields

$$
\begin{aligned}
g_{h z z}^{(1)}= & \frac{-g^{2} v}{192 \pi^{2} m_{H^{ \pm}}^{2}}\left[\frac{g_{0}+g_{A}}{2 c_{W}^{2}}+g_{+}\left(c_{W}^{2}-2 s_{W}^{2}\right)+\left(1-x_{0}\right) \frac{4 g_{0}+g_{+}}{10}\right. \\
& \left.+\left(1-x_{A}\right) \frac{4 g_{A}+g_{+}}{10}\right] \\
g_{h z z}^{(2,6)}= & \frac{g_{h z z}^{(4)}}{2}=\frac{2 g_{h z z}^{(5)}}{5}=\frac{g^{2} v}{192 \pi^{2} m_{H^{ \pm}}^{2}}\left[\frac{\left(x_{A}-x_{0}\right)\left(g_{0}-g_{A}\right)}{40 c_{W}^{2}}\right] \\
g_{h z z}^{(3)}= & \frac{g^{2} v}{192 \pi^{2} c_{W}^{2}}\left[\left(x_{0}-x_{A}\right)\left(g_{0}-g_{A}\right)\right]
\end{aligned}
$$


while for $\Delta \mathcal{L}_{\gamma}$ we obtain

$$
g_{h \gamma \gamma}^{(1)}=\frac{g^{2} v s_{W}^{2} g_{+}}{64 \pi^{2} m_{H^{ \pm}}^{2}}, \quad g_{h \gamma z}^{(1)}=\frac{g^{2} v s_{W}\left(c_{W}^{2}-s_{W}^{2}\right) g_{+}}{128 \pi^{2} m_{H^{ \pm}}^{2} c_{W}}, \quad g_{h \gamma z}^{(2)}=0
$$

Use of (B.15) in (B.19)-(B.22) yields

$$
\begin{aligned}
g_{h z z}^{(1)} & =g_{h w w}^{(1)}-\frac{g^{2} v}{192 \pi^{2} m_{H^{ \pm}}^{2}}\left[\frac{\left(-2 \tilde{\lambda}_{3}+\tilde{\lambda}_{4}\right) s_{W}^{2}}{c_{W}^{2}}+\frac{3 \tilde{\lambda}_{3} s_{W}^{4}}{c_{W}^{2}}\right] \\
g_{h z z}^{(2,4-6)} & \sim \mathcal{O}\left[\left(1-x_{0}\right)^{2},\left(1-x_{A}\right)^{2}\right] \\
g_{h z z}^{(3)} & =\frac{g^{2} v}{192 \pi^{2} c_{W}^{2}}\left[2\left(x_{0}-x_{A}\right) \tilde{\lambda}_{5}\right] \\
g_{h \gamma \gamma}^{(1)} & =g_{h \gamma z}^{(1)} t_{2 W}=\frac{g^{2} v s_{W}^{2} \tilde{\lambda}_{3}}{64 \pi^{2} m_{H^{ \pm}}^{2}} \\
g_{h \gamma z}^{(2)} & =0
\end{aligned}
$$

Altogether, these results show that $g_{h V V}^{(2,4-6)} \sim v^{4} / \tilde{\mu}_{2}^{4}$ at least, and only receive contributions from $D=8$ effective operators. For the $D=6$ effective operators, we use the leading order in (B.16)-(B.18) and (B.23)-(B.27), and through the relations from table 5 we get

$$
\begin{aligned}
& \bar{c}_{\gamma}=\frac{m_{W}^{2} \tilde{\lambda}_{3}}{256 \pi^{2} m_{H^{ \pm}}^{2}}, \quad \bar{c}_{W}=-\bar{c}_{H W}=\frac{m_{W}^{2}\left(2 \tilde{\lambda}_{3}+\tilde{\lambda}_{4}\right)}{192 \pi^{2} m_{H^{ \pm}}^{2}} \\
& \bar{c}_{B}=-\bar{c}_{H B}=\frac{m_{W}^{2}\left(-2 \tilde{\lambda}_{3}+\tilde{\lambda}_{4}\right)}{192 \pi^{2} m_{H^{ \pm}}^{2}}, \quad \bar{c}_{W}-\bar{c}_{B}=-\left(\bar{c}_{H W}-\bar{c}_{H B}\right)=\frac{8}{3} \bar{c}_{\gamma} \\
& \bar{c}_{T} \equiv \frac{1}{2 g m_{W}}\left(g_{h w w}^{(3)}-c_{W}^{2} g_{h z z}^{(3)}\right)=\left(\tilde{\lambda}_{4}^{2}-\tilde{\lambda}_{5}^{2}\right) \frac{v^{2}}{192 \pi^{2} m_{H^{ \pm}}^{2}}
\end{aligned}
$$

which reproduce the results from (3.28) upon the substitution $m_{H^{ \pm}}^{2} \rightarrow \tilde{\mu}_{2}^{2}$.

Open Access. This article is distributed under the terms of the Creative Commons Attribution License (CC-BY 4.0), which permits any use, distribution and reproduction in any medium, provided the original author(s) and source are credited.

\section{References}

[1] ATLAS collaboration, Observation of a new particle in the search for the Standard Model Higgs boson with the ATLAS detector at the LHC, Phys. Lett. B 716 (2012) 1 [arXiv:1207.7214] [INSPIRE].

[2] CMS collaboration, Observation of a new boson at a mass of $125 \mathrm{GeV}$ with the CMS experiment at the LHC, Phys. Lett. B 716 (2012) 30 [arXiv:1207.7235] [InSPIRE].

[3] P.W. Higgs, Broken symmetries and the masses of gauge bosons, Phys. Rev. Lett. 13 (1964) 508 [INSPIRE].

[4] P.W. Higgs, Broken symmetries, massless particles and gauge fields, Phys. Lett. 12 (1964) 132 [INSPIRE]. 
[5] P.W. Higgs, Spontaneous symmetry breakdown without massless bosons, Phys. Rev. 145 (1966) 1156 [INSPIRE].

[6] S. Weinberg, A model of leptons, Phys. Rev. Lett. 19 (1967) 1264 [InSPIRE].

[7] C.J.C. Burges and H.J. Schnitzer, Virtual effects of excited quarks as probes of a possible new hadronic mass scale, Nucl. Phys. B 228 (1983) 464 [INSPIRE].

[8] C.N. Leung, S.T. Love and S. Rao, Low-energy manifestations of a new interaction scale: operator analysis, Z. Phys. C 31 (1986) 433 [InSPIRE].

[9] W. Buchmüller and D. Wyler, Effective Lagrangian analysis of new interactions and flavor conservation, Nucl. Phys. B 268 (1986) 621 [InSPIRE].

[10] K. Hagiwara, S. Ishihara, R. Szalapski and D. Zeppenfeld, Low-energy effects of new interactions in the electroweak boson sector, Phys. Rev. D 48 (1993) 2182 [InSPIRE].

[11] G.F. Giudice, C. Grojean, A. Pomarol and R. Rattazzi, The strongly-interacting light Higgs, JHEP 06 (2007) 045 [hep-ph/0703164] [INSPIRE].

[12] B. Grzadkowski, M. Iskrzynski, M. Misiak and J. Rosiek, Dimension-six terms in the Standard Model Lagrangian, JHEP 10 (2010) 085 [arXiv:1008.4884] [INSPIRE].

[13] T. Corbett, O.J.P. Eboli, J. Gonzalez-Fraile and M.C. Gonzalez-Garcia, Constraining anomalous Higgs interactions, Phys. Rev. D 86 (2012) 075013 [arXiv:1207.1344] [INSPIRE].

[14] J.R. Espinosa, C. Grojean, V. Sanz and M. Trott, NSUSY fits, JHEP 12 (2012) 077 [arXiv:1207.7355] [INSPIRE].

[15] E. Massó and V. Sanz, Limits on anomalous couplings of the Higgs boson to electroweak gauge bosons from LEP and the LHC, Phys. Rev. D 87 (2013) 033001 [arXiv:1211.1320] [INSPIRE].

[16] T. Corbett, O.J.P. Eboli, J. Gonzalez-Fraile and M.C. Gonzalez-Garcia, Robust determination of the Higgs couplings: power to the data, Phys. Rev. D 87 (2013) 015022 [arXiv: 1211.4580] [INSPIRE].

[17] B. Dumont, S. Fichet and G. von Gersdorff, A Bayesian view of the Higgs sector with higher dimensional operators, JHEP 07 (2013) 065 [arXiv: 1304.3369] [INSPIRE].

[18] A. Pomarol and F. Riva, Towards the ultimate SM fit to close in on Higgs physics, JHEP 01 (2014) 151 [arXiv: 1308.2803] [INSPIRE].

[19] J. Ellis, V. Sanz and T. You, Complete Higgs sector constraints on dimension-6 operators, JHEP 07 (2014) 036 [arXiv:1404.3667] [InSPIRE].

[20] R.S. Gupta, A. Pomarol and F. Riva, BSM primary effects, Phys. Rev. D 91 (2015) 035001 [arXiv: 1405.0181] [INSPIRE].

[21] J. Ellis, V. Sanz and T. You, The effective Standard Model after LHC Run I, JHEP 03 (2015) 157 [arXiv: 1410.7703] [INSPIRE].

[22] A. Biekötter, A. Knochel, M. Krämer, D. Liu and F. Riva, Vices and virtues of Higgs effective field theories at large energy, Phys. Rev. D 91 (2015) 055029 [arXiv:1406.7320] [INSPIRE].

[23] C. Englert and M. Spannowsky, Effective theories and measurements at colliders, Phys. Lett. B 740 (2015) 8 [arXiv:1408.5147] [INSPIRE]. 
[24] S. Willenbrock and C. Zhang, Effective field theory beyond the Standard Model, Ann. Rev. Nucl. Part. Sci. 64 (2014) 83 [arXiv:1401.0470] [INSPIRE].

[25] R. Contino, M. Ghezzi, C. Grojean, M. Muhlleitner and M. Spira, Effective Lagrangian for a light Higgs-like scalar, JHEP 07 (2013) 035 [arXiv:1303.3876] [INSPIRE].

[26] A. Alloul, B. Fuks and V. Sanz, Phenomenology of the Higgs effective Lagrangian via FEYNRULES, JHEP 04 (2014) 110 [arXiv: 1310.5150] [INSPIRE].

[27] M. Gorbahn, J.M. No and V. Sanz, in preparation.

[28] J. Elias-Miro, J.R. Espinosa, E. Masso and A. Pomarol, Higgs windows to new physics through $D=6$ operators: constraints and one-loop anomalous dimensions, JHEP 11 (2013) 066 [arXiv:1308.1879] [INSPIRE].

[29] E.E. Jenkins, A.V. Manohar and M. Trott, Renormalization group evolution of the Standard Model dimension six operators I: formalism and $\lambda$ dependence, JHEP 10 (2013) 087 [arXiv: 1308.2627] [INSPIRE].

[30] E.E. Jenkins, A.V. Manohar and M. Trott, Renormalization group evolution of the Standard Model dimension six operators II: Yukawa dependence, JHEP 01 (2014) 035 [arXiv:1310.4838] [INSPIRE].

[31] R. Alonso, E.E. Jenkins, A.V. Manohar and M. Trott, Renormalization group evolution of the Standard Model dimension six operators III: gauge coupling dependence and phenomenology, JHEP 04 (2014) 159 [arXiv:1312.2014] [INSPIRE].

[32] J. de Blas, M. Chala, M. Pérez-Victoria and J. Santiago, Observable effects of general new scalar particles, JHEP 04 (2015) 078 [arXiv: 1412.8480] [INSPIRE].

[33] A.J. Buras, Weak Hamiltonian, CP-violation and rare decays, hep-ph/9806471 [INSPIRE].

[34] M. Gorbahn, S. Jager, U. Nierste and S. Trine, The supersymmetric Higgs sector and B- $\bar{B}$ mixing for large $\tan \beta$, Phys. Rev. D 84 (2011) 034030 [arXiv:0901.2065] [INSPIRE].

[35] O. Lebedev, On stability of the electroweak vacuum and the Higgs portal, Eur. Phys. J. C 72 (2012) 2058 [arXiv: 1203.0156] [INSPIRE].

[36] J. Elias-Miro, J.R. Espinosa, G.F. Giudice, H.M. Lee and A. Strumia, Stabilization of the electroweak vacuum by a scalar threshold effect, JHEP 06 (2012) 031 [arXiv:1203.0237] [INSPIRE].

[37] R. Schabinger and J.D. Wells, A minimal spontaneously broken hidden sector and its impact on Higgs boson physics at the Large Hadron Collider, Phys. Rev. D 72 (2005) 093007 [hep-ph/0509209] [INSPIRE].

[38] B. Patt and F. Wilczek, Higgs-field portal into hidden sectors, hep-ph/0605188 [INSPIRE].

[39] V. Silveira and A. Zee, Scalar phantoms, Phys. Lett. B 161 (1985) 136 [inSPIRE].

[40] J. McDonald, Gauge singlet scalars as cold dark matter, Phys. Rev. D 50 (1994) 3637 [hep-ph/0702143] [INSPIRE].

[41] D. O'Connell, M.J. Ramsey-Musolf and M.B. Wise, Minimal extension of the Standard Model scalar sector, Phys. Rev. D 75 (2007) 037701 [hep-ph/0611014] [INSPIRE].

[42] G.W. Anderson and L.J. Hall, The electroweak phase transition and baryogenesis, Phys. Rev. D 45 (1992) 2685 [INSPIRE]. 
[43] J.R. Espinosa and M. Quirós, The electroweak phase transition with a singlet, Phys. Lett. B 305 (1993) 98 [hep-ph/9301285] [INSPIRE].

[44] S. Profumo, M.J. Ramsey-Musolf and G. Shaughnessy, Singlet Higgs phenomenology and the electroweak phase transition, JHEP 08 (2007) 010 [arXiv:0705.2425] [INSPIRE].

[45] J.R. Espinosa, T. Konstandin, J.M. No and M. Quirós, Some cosmological implications of hidden sectors, Phys. Rev. D 78 (2008) 123528 [arXiv:0809.3215] [InSPIRE].

[46] J.R. Espinosa, T. Konstandin and F. Riva, Strong electroweak phase transitions in the Standard Model with a singlet, Nucl. Phys. B 854 (2012) 592 [arXiv:1107.5441] [INSPIRE].

[47] S. Profumo, M.J. Ramsey-Musolf, C.L. Wainwright and P. Winslow, Singlet-catalyzed electroweak phase transitions and precision Higgs boson studies, Phys. Rev. D 91 (2015) 035018 [arXiv: 1407.5342] [INSPIRE].

[48] A. Farzinnia and J. Ren, Strongly first-order electroweak phase transition and classical scale invariance, Phys. Rev. D 90 (2014) 075012 [arXiv:1408.3533] [InSPIRE].

[49] D. Curtin, P. Meade and C.-T. Yu, Testing electroweak baryogenesis with future colliders, JHEP 11 (2014) 127 [arXiv:1409.0005] [InSPIRE].

[50] V. Barger, P. Langacker, M. McCaskey, M.J. Ramsey-Musolf and G. Shaughnessy, LHC phenomenology of an extended Standard Model with a real scalar singlet, Phys. Rev. D 77 (2008) 035005 [arXiv:0706.4311] [INSPIRE].

[51] M.J. Dolan, C. Englert and M. Spannowsky, New physics in LHC Higgs boson pair production, Phys. Rev. D 87 (2013) 055002 [arXiv:1210.8166] [INSPIRE].

[52] J.M. No and M. Ramsey-Musolf, Probing the Higgs portal at the LHC through resonant di-Higgs production, Phys. Rev. D 89 (2014) 095031 [arXiv:1310.6035] [INSPIRE].

[53] A. Efrati and Y. Nir, What if $\lambda_{h h h} \neq 3 m_{h}^{2} / v$, arXiv:1401.0935 [INSPIRE].

[54] G.M. Pruna and T. Robens, Higgs singlet extension parameter space in the light of the LHC discovery, Phys. Rev. D 88 (2013) 115012 [arXiv: 1303.1150] [INSPIRE].

[55] T. Robens and T. Stefaniak, Status of the Higgs singlet extension of the Standard Model after LHC Run 1, Eur. Phys. J. C 75 (2015) 104 [arXiv:1501.02234] [InSPIRE].

[56] V. Martín Lozano, J.M. Moreno and C.B. Park, Resonant Higgs boson pair production in the $h h \rightarrow b \bar{b} W W \rightarrow b \bar{b} \ell^{+} \nu \ell^{-} \bar{\nu}$ decay channel, JHEP 08 (2015) 004 [arXiv:1501.03799] [INSPIRE].

[57] A. Falkowski, C. Gross and O. Lebedev, A second Higgs from the Higgs portal, JHEP 05 (2015) 057 [arXiv: 1502.01361] [InSPIRE].

[58] M. Gouzevitch, A. Oliveira, J. Rojo, R. Rosenfeld, G.P. Salam and V. Sanz, Scale-invariant resonance tagging in multijet events and new physics in Higgs pair production, JHEP $\mathbf{0 7}$ (2013) 148 [arXiv:1303.6636] [InSPIRE].

[59] C.-Y. Chen, S. Dawson and I.M. Lewis, Exploring resonant di-Higgs boson production in the Higgs singlet model, Phys. Rev. D 91 (2015) 035015 [arXiv:1410.5488] [INSPIRE].

[60] N. Craig, C. Englert and M. McCullough, New probe of naturalness, Phys. Rev. Lett. 111 (2013) 121803 [arXiv:1305.5251] [inSPIRE].

[61] ATLAS collaboration, Measurement of Higgs boson production in the diphoton decay channel in pp collisions at center-of-mass energies of 7 and $8 \mathrm{TeV}$ with the ATLAS detector, Phys. Rev. D 90 (2014) 112015 [arXiv: 1408.7084] [INSPIRE]. 
[62] CMS collaboration, Observation of the diphoton decay of the Higgs boson and measurement of its properties, Eur. Phys. J. C 74 (2014) 3076 [arXiv: 1407.0558] [INSPIRE].

[63] ATLAS collaboration, Measurements of Higgs boson production and couplings in the four-lepton channel in pp collisions at center-of-mass energies of 7 and $8 \mathrm{TeV}$ with the ATLAS detector, Phys. Rev. D 91 (2015) 012006 [arXiv:1408.5191] [INSPIRE].

[64] CMS collaboration, Measurement of the properties of a Higgs boson in the four-lepton final state, Phys. Rev. D 89 (2014) 092007 [arXiv: 1312.5353] [InSPIRE].

[65] ATLAS collaboration, Measurements of the properties of the Higgs-like boson in the $W W^{(*)} \rightarrow \ell \nu \ell \nu$ decay channel with the ATLAS detector using $25 \mathrm{fb}^{-1}$ of proton-proton collision data, ATLAS-CONF-2013-030, CERN, Geneva Switzerland (2013).

[66] CMS collaboration, Measurement of Higgs boson production and properties in the $W W$ decay channel with leptonic final states, JHEP 01 (2014) 096 [arXiv:1312.1129] [INSPIRE].

[67] ATLAS collaboration, Search for the bb decay of the Standard Model Higgs boson in associated W/ZH production with the ATLAS detector, ATLAS-CONF-2013-079, CERN, Geneva Switzerland (2013).

[68] CMS collaboration, Search for the Standard Model Higgs boson produced in association with $a W$ or a $Z$ boson and decaying to bottom quarks, Phys. Rev. D 89 (2014) 012003 [arXiv:1310.3687] [INSPIRE].

[69] ATLAS collaboration, Evidence for Higgs boson decays to the $\tau^{+} \tau^{-}$final state with the ATLAS detector, ATLAS-CONF-2013-108, CERN, Geneva Switzerland (2013).

[70] CMS collaboration, Evidence for the $125 \mathrm{GeV}$ Higgs boson decaying to a pair of $\tau$ leptons, JHEP 05 (2014) 104 [arXiv: 1401.5041] [INSPIRE].

[71] CMS collaboration, Projected performance of an upgraded CMS detector at the LHC and HL-LHC: contribution to the Snowmass process, arXiv:1307.7135 [INSPIRE].

[72] GFitTer Group collaboration, M. Baak et al., The global electroweak fit at NNLO and prospects for the LHC and ILC, Eur. Phys. J. C 74 (2014) 3046 [arXiv: 1407.3792] [INSPIRE].

[73] K. Hagiwara, S. Matsumoto, D. Haidt and C.S. Kim, A novel approach to confront electroweak data and theory, Z. Phys. C 64 (1994) 559 [Erratum ibid. C 68 (1995) 352] [hep-ph/9409380] [INSPIRE].

[74] J. Fan, M. Reece and L.-T. Wang, Possible futures of electroweak precision: ILC, FCC-ee and $C E P C$, arXiv: 1411.1054 [INSPIRE].

[75] TLeP Design Study Working Group collaboration, M. Bicer et al., First look at the physics case of TLEP, JHEP 01 (2014) 164 [arXiv: 1308.6176] [INSPIRE].

[76] S. Mishima, Sensitivity to new physics from TLEP precision measurements, contribution at $6^{\text {th }}$ TLEP workshop, http://indico.cern.ch/event/257713/session/1/contribution/30, CERN, Geneva Switzerland October 162013.

[77] D.M. Asner et al., ILC Higgs white paper, arXiv:1310.0763 [INSPIRE].

[78] B. Henning, X. Lu and H. Murayama, What do precision Higgs measurements buy us?, arXiv: 1404.1058 [INSPIRE].

[79] J. Elias-Miró, C. Grojean, R.S. Gupta and D. Marzocca, Scaling and tuning of EW and Higgs observables, JHEP 05 (2014) 019 [arXiv:1312.2928] [INSPIRE]. 
[80] CMS collaboration, Search for a Standard Model like Higgs boson in the decay channel $H \rightarrow Z Z \rightarrow \ell^{+} \ell^{-} q \bar{q}$ at $C M S$, CMS-PAS-HIG-12-024, CERN, Geneva Switzerland (2012).

[81] CMS collaboration, Search for the Standard Model Higgs boson in the $H \rightarrow W W \rightarrow \ell \nu j j$ decay channel in pp collisions at the LHC, CMS-PAS-HIG-13-027, CERN, Geneva Switzerland (2013).

[82] C. Grojean, G. Servant and J.D. Wells, First-order electroweak phase transition in the Standard Model with a low cutoff, Phys. Rev. D 71 (2005) 036001 [hep-ph/0407019] [INSPIRE].

[83] D. Bödeker, L. Fromme, S.J. Huber and M. Seniuch, The baryon asymmetry in the Standard Model with a low cut-off, JHEP 02 (2005) 026 [hep-ph/0412366] [INSPIRE].

[84] C. Delaunay, C. Grojean and J.D. Wells, Dynamics of non-renormalizable electroweak symmetry breaking, JHEP 04 (2008) 029 [arXiv:0711.2511] [INSPIRE].

[85] A. Djouadi, The anatomy of electro-weak symmetry breaking. II. The Higgs bosons in the minimal supersymmetric model, Phys. Rept. 459 (2008) 1 [hep-ph/0503173] [INSPIRE].

[86] U. Ellwanger, C. Hugonie and A.M. Teixeira, The next-to-minimal supersymmetric Standard Model, Phys. Rept. 496 (2010) 1 [arXiv:0910.1785] [InSPIRE].

[87] R. Barbieri, L.J. Hall and V.S. Rychkov, Improved naturalness with a heavy Higgs: an alternative road to LHC physics, Phys. Rev. D 74 (2006) 015007 [hep-ph/0603188] [INSPIRE].

[88] L. Lopez Honorez, E. Nezri, J.F. Oliver and M.H.G. Tytgat, The inert doublet model: an archetype for dark matter, JCAP 02 (2007) 028 [hep-ph/0612275] [INSPIRE].

[89] E.M. Dolle and S. Su, The inert dark matter, Phys. Rev. D 80 (2009) 055012 [arXiv: 0906.1609] [INSPIRE].

[90] E. Ma, Verifiable radiative seesaw mechanism of neutrino mass and dark matter, Phys. Rev. D 73 (2006) 077301 [hep-ph/0601225] [INSPIRE].

[91] M. Aoki, S. Kanemura and O. Seto, Neutrino mass, dark matter and baryon asymmetry via TeV-scale physics without fine-tuning, Phys. Rev. Lett. 102 (2009) 051805 [arXiv:0807.0361] [INSPIRE].

[92] M. Gustafsson, J.M. No and M.A. Rivera, Predictive model for radiatively induced neutrino masses and mixings with dark matter, Phys. Rev. Lett. 110 (2013) 211802 [Erratum ibid. 112 (2014) 259902] [arXiv: 1212.4806] [INSPIRE].

[93] G.C. Branco, P.M. Ferreira, L. Lavoura, M.N. Rebelo, M. Sher and J.P. Silva, Theory and phenomenology of two-Higgs-doublet models, Phys. Rept. 516 (2012) 1 [arXiv:1106.0034] [INSPIRE].

[94] A. Celis, V. Ilisie and A. Pich, LHC constraints on two-Higgs doublet models, JHEP 07 (2013) 053 [arXiv: 1302.4022] [InSPIRE].

[95] M. Krawczyk, D. Sokołowska and B. Świeżewska, $2 H D M$ with $Z_{2}$ symmetry in light of new LHC data, J. Phys. Conf. Ser. 447 (2013) 012050 [arXiv:1303.7102] [INSPIRE].

[96] B. Grinstein and P. Uttayarat, Carving out parameter space in type-II two Higgs doublets model, JHEP 06 (2013) 094 [Erratum ibid. 09 (2013) 110] [arXiv:1304.0028] [INSPIRE].

[97] C.-Y. Chen, S. Dawson and M. Sher, Heavy Higgs searches and constraints on two Higgs doublet models, Phys. Rev. D 88 (2013) 015018 [Erratum ibid. D 88 (2013) 039901] [arXiv: 1305.1624] [INSPIRE]. 
[98] O. Eberhardt, U. Nierste and M. Wiebusch, Status of the two-Higgs-doublet model of type-II, JHEP 07 (2013) 118 [arXiv: 1305.1649] [INSPIRE].

[99] G. Bélanger, B. Dumont, U. Ellwanger, J.F. Gunion and S. Kraml, Global fit to Higgs signal strengths and couplings and implications for extended Higgs sectors, Phys. Rev. D 88 (2013) 075008 [arXiv: 1306.2941] [INSPIRE].

[100] K. Cheung, J.S. Lee and P.-Y. Tseng, Higgcision in the two-Higgs doublet models, JHEP 01 (2014) 085 [arXiv: 1310.3937] [inSPIRE].

[101] B. Dumont, J.F. Gunion, Y. Jiang and S. Kraml, Constraints on and future prospects for two-Higgs-doublet models in light of the LHC Higgs signal, Phys. Rev. D 90 (2014) 035021 [arXiv: 1405.3584] [INSPIRE].

[102] S. Kanemura, K. Tsumura, K. Yagyu and H. Yokoya, Fingerprinting nonminimal Higgs sectors, Phys. Rev. D 90 (2014) 075001 [arXiv: 1406.3294] [INSPIRE].

[103] J.M. Cline and P.-A. Lemieux, Electroweak phase transition in two Higgs doublet models, Phys. Rev. D 55 (1997) 3873 [hep-ph/9609240] [INSPIRE].

[104] S. Kanemura, Y. Okada and E. Senaha, Electroweak baryogenesis and quantum corrections to the triple Higgs boson coupling, Phys. Lett. B 606 (2005) 361 [hep-ph/0411354] [INSPIRE].

[105] L. Fromme, S.J. Huber and M. Seniuch, Baryogenesis in the two-Higgs doublet model, JHEP 11 (2006) 038 [hep-ph/0605242] [INSPIRE].

[106] G.C. Dorsch, S.J. Huber and J.M. No, A strong electroweak phase transition in the $2 H D M$ after LHC8, JHEP 10 (2013) 029 [arXiv: 1305.6610] [INSPIRE].

[107] S. Kanemura, S. Moretti, Y. Mukai, R. Santos and K. Yagyu, Distinctive Higgs signals of a type II 2 HDM at the LHC, Phys. Rev. D 79 (2009) 055017 [arXiv:0901.0204] [INSPIRE].

[108] N. Craig, J. Galloway and S. Thomas, Searching for signs of the second Higgs doublet, arXiv:1305.2424 [INSPIRE].

[109] J. Baglio, O. Eberhardt, U. Nierste and M. Wiebusch, Benchmarks for Higgs pair production and heavy Higgs boson searches in the two-Higgs-doublet model of type II, Phys. Rev. D 90 (2014) 015008 [arXiv: 1403.1264] [INSPIRE].

[110] B. Coleppa, F. Kling and S. Su, Exotic decays of a heavy neutral Higgs through HZ/AZ channel, JHEP 09 (2014) 161 [arXiv: 1404.1922] [INSPIRE].

[111] G.C. Dorsch, S.J. Huber, K. Mimasu and J.M. No, Echoes of the electroweak phase transition: discovering a second Higgs doublet through $A_{0} \rightarrow Z H_{0}$, Phys. Rev. Lett. 113 (2014) 211802 [arXiv:1405.5537] [INSPIRE].

[112] B. Hespel, D. Lopez-Val and E. Vryonidou, Higgs pair production via gluon fusion in the two-Higgs-doublet model, JHEP 09 (2014) 124 [arXiv:1407.0281] [INSPIRE].

[113] W. Altmannshofer, S. Gori and G.D. Kribs, A minimal flavor violating $2 H D M$ at the LHC, Phys. Rev. D 86 (2012) 115009 [arXiv: 1210.2465] [INSPIRE].

[114] S.L. Glashow and S. Weinberg, Natural conservation laws for neutral currents, Phys. Rev. D 15 (1977) 1958 [INSPIRE].

[115] C. Englert et al., Precision measurements of Higgs couplings: implications for new physics scales, J. Phys. G 41 (2014) 113001 [arXiv:1403.7191] [INSPIRE]. 
[116] J.F. Gunion and H.E. Haber, The CP conserving two Higgs doublet model: the approach to the decoupling limit, Phys. Rev. D 67 (2003) 075019 [hep-ph/0207010] [INSPIRE].

[117] M. Carena, I. Low, N.R. Shah and C.E.M. Wagner, Impersonating the Standard Model Higgs boson: alignment without decoupling, JHEP 04 (2014) 015 [arXiv:1310.2248] [INSPIRE].

[118] W. Grimus, L. Lavoura, O.M. Ogreid and P. Osland, The oblique parameters in multi-Higgs-doublet models, Nucl. Phys. B 801 (2008) 81 [arXiv:0802.4353] [INSPIRE].

[119] G. Altarelli and R. Barbieri, Vacuum polarization effects of new physics on electroweak processes, Phys. Lett. B 253 (1991) 161 [INSPIRE].

[120] G. Altarelli, R. Barbieri and S. Jadach, Toward a model independent analysis of electroweak data, Nucl. Phys. B 369 (1992) 3 [Erratum ibid. B 376 (1992) 444] [INSPIRE].

[121] R. Barbieri, A. Pomarol, R. Rattazzi and A. Strumia, Electroweak symmetry breaking after LEP-1 and LEP-2, Nucl. Phys. B 703 (2004) 127 [hep-ph/0405040] [INSPIRE].

[122] J. Hirn and V. Sanz, (Not) summing over Kaluza-Kleins, Phys. Rev. D 76 (2007) 044022 [hep-ph/0702005] [INSPIRE].

[123] J. Hirn and V. Sanz, A negative S parameter from holographic technicolor, Phys. Rev. Lett. 97 (2006) 121803 [hep-ph/0606086] [INSPIRE].

[124] J. Hirn and V. Sanz, The fifth dimension as an analogue computer for strong interactions at the LHC, JHEP 03 (2007) 100 [hep-ph/0612239] [INSPIRE].

[125] C. Csáki, J. Hubisz and S.J. Lee, Radion phenomenology in realistic warped space models, Phys. Rev. D 76 (2007) 125015 [arXiv:0705.3844] [INSPIRE].

[126] H.M. Lee, M. Park and V. Sanz, Gravity-mediated (or composite) dark matter, Eur. Phys. J. C 74 (2014) 2715 [arXiv: 1306.4107] [INSPIRE].

[127] H.M. Lee, M. Park and V. Sanz, Gravity-mediated (or composite) dark matter confronts astrophysical data, JHEP 05 (2014) 063 [arXiv:1401.5301] [INSPIRE].

[128] W.D. Goldberger, B. Grinstein and W. Skiba, Distinguishing the Higgs boson from the dilaton at the Large Hadron Collider, Phys. Rev. Lett. 100 (2008) 111802 [arXiv:0708.1463] [INSPIRE].

[129] W.D. Goldberger and M.B. Wise, Modulus stabilization with bulk fields, Phys. Rev. Lett. 83 (1999) 4922 [hep-ph/9907447] [INSPIRE].

[130] W.D. Goldberger and M.B. Wise, Phenomenology of a stabilized modulus, Phys. Lett. B 475 (2000) 275 [hep-ph/9911457] [INSPIRE].

[131] C. Csáki, M.L. Graesser and G.D. Kribs, Radion dynamics and electroweak physics, Phys. Rev. D 63 (2001) 065002 [hep-th/0008151] [INSPIRE].

[132] J.F. Gunion, M. Toharia and J.D. Wells, Precision electroweak data and the mixed radion-Higgs sector of warped extra dimensions, Phys. Lett. B 585 (2004) 295 [hep-ph/0311219] [INSPIRE].

[133] J. Ellis, D.S. Hwang, V. Sanz and T. You, A fast track towards the 'Higgs' spin and parity, JHEP 11 (2012) 134 [arXiv:1208.6002] [INSPIRE].

[134] J. Ellis, V. Sanz and T. You, Associated production evidence against Higgs impostors and anomalous couplings, Eur. Phys. J. C 73 (2013) 2507 [arXiv: 1303.0208] [INSPIRE]. 
[135] J.M. Campbell, $W / Z+B, \bar{B} /$ jets at $N L O$ using the Monte Carlo MCFM, hep-ph/0105226 [INSPIRE].

[136] R.K. Ellis, An update on the next-to-leading order Monte Carlo MCFM, Nucl. Phys. Proc. Suppl. 160 (2006) 170 [INSPIRE].

[137] J.M. Campbell and R.K. Ellis, MCFM for the Tevatron and the LHC, Nucl. Phys. Proc. Suppl. 205-206 (2010) 10 [arXiv: 1007.3492] [INSPIRE].

[138] J.M. Campbell, R.K. Ellis and C. Williams, Vector boson pair production at the LHC, JHEP 07 (2011) 018 [arXiv:1105.0020] [INSPIRE].

[139] J.M. Campbell, R.K. Ellis, R. Frederix, P. Nason, C. Oleari and C. Williams, NLO Higgs boson production plus one and two jets using the POWHEG BOX, MadGraph4 and MCFM, JHEP 07 (2012) 092 [arXiv: 1202.5475] [INSPIRE].

[140] E. Bagnaschi, G. Degrassi, P. Slavich and A. Vicini, Higgs production via gluon fusion in the POWHEG approach in the SM and in the MSSM, JHEP 02 (2012) 088 [arXiv:1111.2854] [INSPIRE].

[141] J. Alwall, M. Herquet, F. Maltoni, O. Mattelaer and T. Stelzer, MadGraph 5: going beyond, JHEP 06 (2011) 128 [arXiv:1106.0522] [INSPIRE].

[142] A. Alloul, B. Fuks and V. Sanz, Phenomenology of the Higgs effective Lagrangian via FEYNRULES, JHEP 04 (2014) 110 [arXiv:1310.5150] [INSPIRE].

[143] N.D. Christensen and C. Duhr, FeynRules - Feynman rules made easy, Comput. Phys. Commun. 180 (2009) 1614 [arXiv:0806.4194] [INSPIRE].

[144] C. Degrande, C. Duhr, B. Fuks, D. Grellscheid, O. Mattelaer and T. Reiter, UFO - the Universal FeynRules Output, Comput. Phys. Commun. 183 (2012) 1201 [arXiv:1108.2040] [INSPIRE].

[145] A. Djouadi, R.M. Godbole, B. Mellado and K. Mohan, Probing the spin-parity of the Higgs boson via jet kinematics in vector boson fusion, Phys. Lett. B 723 (2013) 307 [arXiv: 1301.4965] [INSPIRE].

[146] R. Edezhath, Dimension-6 operator constraints from boosted VBF Higgs, arXiv: 1501.00992 [INSPIRE].

[147] G. Brooijmans et al., Les Houches 2013: physics at TeV colliders - new physics working group report, arXiv:1405.1617 [INSPIRE].

[148] S. Kanemura, T. Kubota and H.-A. Tohyama, Generalized screening theorem for Higgs decay processes in the two doublet model, Nucl. Phys. B 483 (1997) 111 [Erratum ibid. B 506 (1997) 548] [hep-ph/9604381] [INSPIRE].

[149] K. Hagiwara, R.D. Peccei, D. Zeppenfeld and K. Hikasa, Probing the weak boson sector in $e^{+} e^{-} \rightarrow W^{+} W^{-}$, Nucl. Phys. B 282 (1987) 253 [inSPIRE].

[150] A. De Rujula, M.B. Gavela, P. Hernández and E. Masso, The selfcouplings of vector bosons: does LEP-1 obviate LEP-2?, Nucl. Phys. B 384 (1992) 3 [inSPIRE].

[151] B. Henning, X. Lu and H. Murayama, How to use the Standard Model effective field theory, arXiv: 1412.1837 [INSPIRE]. 